Summer 2017

\title{
Superconducting Thin Films for The Enhancement of Superconducting Radio Frequency Accelerator Cavities
}

\author{
Matthew Burton \\ College of William and Mary - Arts \& Sciences, mcburton@email.wm.edu
}

Follow this and additional works at: https://scholarworks.wm.edu/etd

Part of the Condensed Matter Physics Commons

\section{Recommended Citation}

Burton, Matthew, "Superconducting Thin Films for The Enhancement of Superconducting Radio Frequency Accelerator Cavities" (2017). Dissertations, Theses, and Masters Projects. Paper 1530192378.

http://dx.doi.org/10.21220/s2-382k-7986

This Dissertation is brought to you for free and open access by the Theses, Dissertations, \& Master Projects at W\&M ScholarWorks. It has been accepted for inclusion in Dissertations, Theses, and Masters Projects by an authorized administrator of W\&M ScholarWorks. For more information, please contact scholarworks@wm.edu. 
Superconducting Thin Films for the Enhancement of Superconducting Radio Frequency Accelerator Cavities

\author{
Matthew C. Burton \\ Chesapeake, Virginia
}

Master of Science, College of William \& Mary, 2014 Bachelor of Science, James Madison University, 2012

A Dissertation presented to the Graduate Faculty of The College of William \& Mary in Candidacy for the Degree of Doctor of Philosophy

Department of Physics

College of William \& Mary January 2018 
(C) Copyright by Matthew C. Burton 2017 
APPROVAL PAGE

This Dissertation is submitted in partial fulfillment of the requirements for the degree of

Doctor of Philosophy

$\frac{\text { Matcher } 23 n \mathbb{Z 1}}{\text { Matthew C. Burton }}$

Approved by the Committee, November 2017

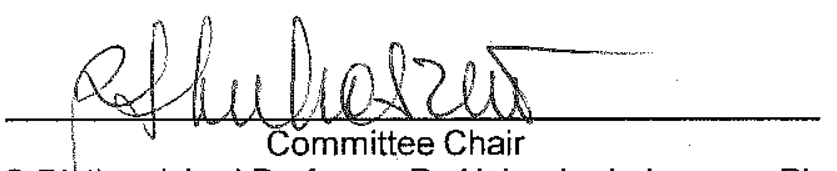

VMEC Distinguished Professor R. Alejandra Lukaszew, Physics College of William \& Mary

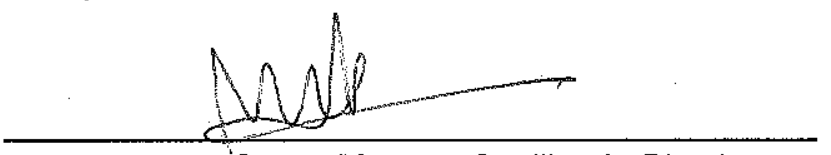

Associate Professor Mumtaz Qazilbash, Physics

College of William \& Mary

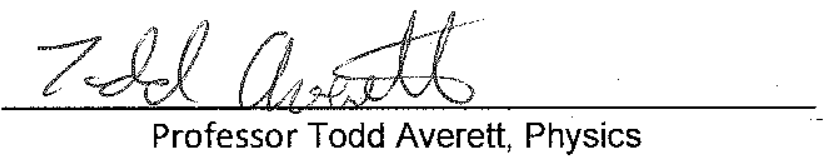

College of William \& Mary

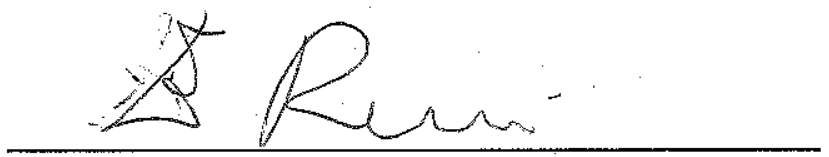

Associate Professor Enrico Rossi, Physics

College of William \& Mary

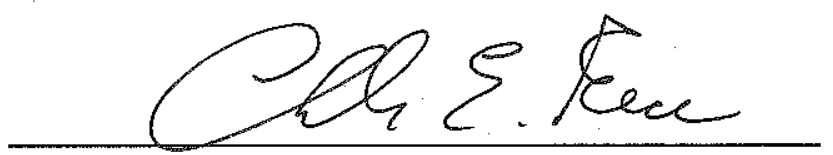

Dr. Charles E. Reece

Jefferson Lab Institute for SRF Science and Technology 


\begin{abstract}
Bulk niobium (Nb) superconducting radio frequency (SRF) cavities are currently the preferred method for acceleration of charged particles at accelerating facilities around the world. However, bulk $\mathrm{Nb}$ cavities have poor thermal conductance, impose material and design restrictions on other components of a particle accelerator, have low reproducibility and are approaching the fundamental material-dependent accelerating field limit of approximately $50 \mathrm{MV} / \mathrm{m}$. Since the SRF phenomena occurs at surfaces within a shallow depth of $\sim 1 \mu \mathrm{m}$, a proposed solution to this problem has been to utilize thin film technology to deposit superconducting thin films on the interior of cavities to engineer the active SRF surface in order to achieve cavities with enhanced properties and performance. Two proposed thin film applications for SRF cavities are: 1) $\mathrm{Nb}$ thin films coated on bulk cavities made of suitable castable metals (such as copper or aluminum) and 2) multilayer films designed to increase the accelerating gradient and performance of SRF cavities. While Nb thin films on copper $(\mathrm{Cu})$ cavities have been attempted in the past using DC magnetron sputtering (DCMS), such cavities have never performed at the bulk Nb level. However, new energetic condensation techniques for film deposition, such as High Power Impulse Magnetron Sputtering (HiPIMS), offer the opportunity to create suitably thick $\mathrm{Nb}$ films with improved density, microstructure and adhesion compared to traditional DCMS. Clearly use of such novel technique requires fundamental studies to assess surface evolution and growth modes during deposition and resulting microstructure and surface morphology and the correlation with RF superconducting properties. Here we present detailed structure-property correlative research studies done on $\mathrm{Nb} / \mathrm{Cu}$ thin films and $\mathrm{NbN}$ - and $\mathrm{NbTiN}$-based multilayers made using HiPIMS and DCMS, respectively.
\end{abstract}




\section{TABLE OF CONTENTS}

Acknowledgements ..........................................................................ii

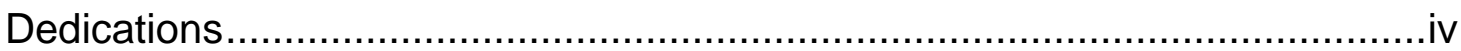

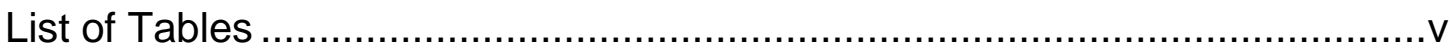

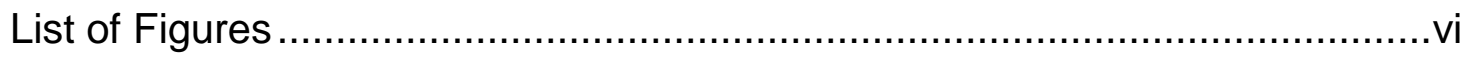

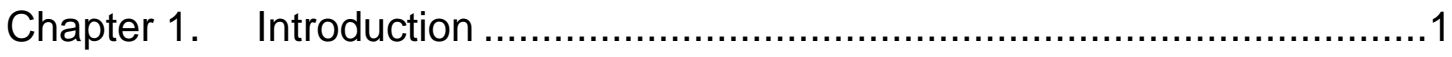

1.1 Accelerator History and Fundamentals .............................

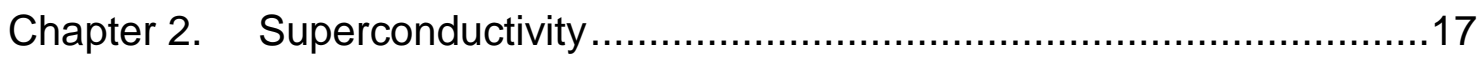

2.1 Meissner Effect and Magnetic Behavior ..............................20

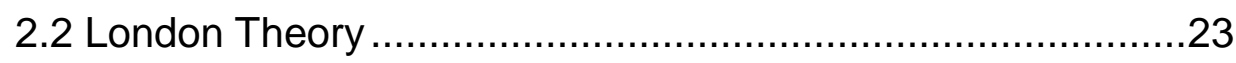

2.3 Ginzburg-Landau Theory ................................................26

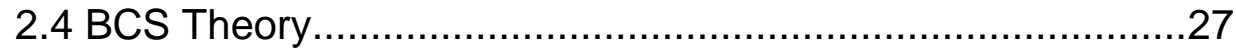

2.5 Surface Resistance of Superconductors .............................30

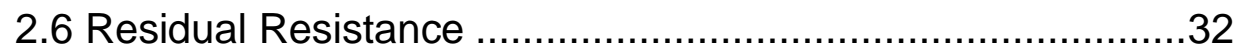

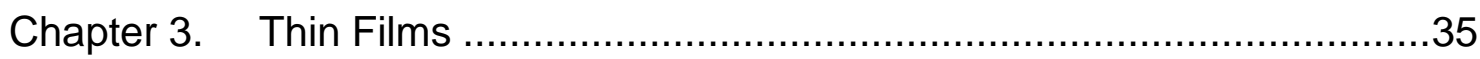

3.1 Thin Film Growth ..........................................................

3.2 Thin Film Structure ........................................................... 41

Chapter 4. Experimental Methods …..................................................46

4.1 Vacuum Technology ................................................... 46

4.1.1 Vacuum Hardware ..............................................47

4.1.2 Vacuum Pumps ................................................... 50

4.1.3 Vacuum Measurement .............................................56

4.2 Thin Film Deposition Techniques .....................................60

4.2.1 Chemical Vapor Deposition......................................61

4.2.2 Physical Vapor Deposition .........................................63 


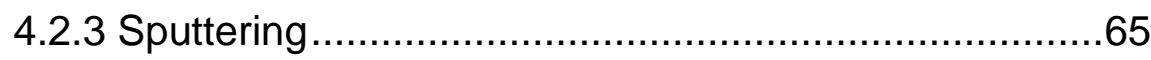

4.3 Film Characterization Techniques ....................................76

4.3.1 Diffraction Techniques.................................................77

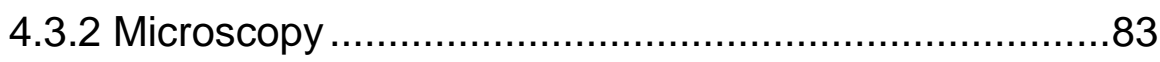

4.3.3 Superconducting Property Measurement .....................88

Chapter 5. $\quad \mathrm{NbN} \& \mathrm{NbTiN}$ Thin Films for SIS Multilayers ..............................97

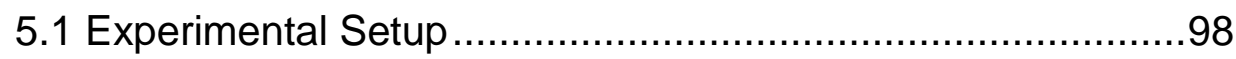

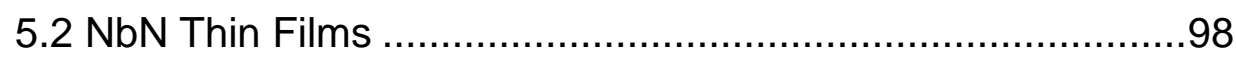

5.2.1 NbN-Based Multilayers............................................101

5.3 NbTiN Thin Films …....................................................103

Chapter 6. $\mathrm{Nb} / \mathrm{Cu}$ Thin Film SRF Cavities ….........................................112

6.1 Experimental Setup .........................................................112

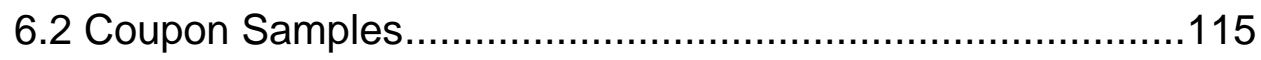

6.3 Cavity RF Results .........................................................122

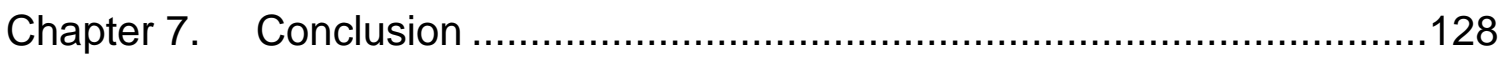

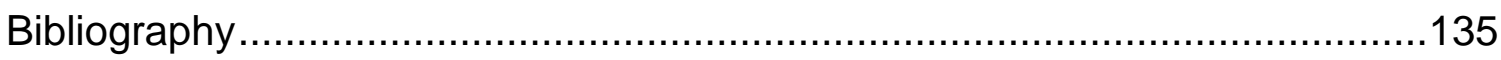




\section{ACKNOWLEDGEMENTS}

First, I would like to express my thanks to my advisors Ale Lukaszew and Larry Phillips. They have always supported me and urged me on to ask better questions, think about things more deeply and not settle for what is easy. Larry, you will be missed. I would also like to express my gratitude to the other students in the Lukaszew research group - Melissa Beebe, Doug Beringer, Kaida Yang, Zhaozhu $\mathrm{Li}$, Scott Madaras and Lei Wang - for their selfless willingness to help teach and give guidance, destress and talk through problems and their support.

I would like to thank my family and friends. My parents for their unfailing support throughout my entire life, making it possible for me to pursue this level of education, their love through all the ups and downs of life, their constant motivation to not settle for the easy path in life and aspire to more and for their willingness to listen to me vent on the phone, especially my mom. To my brothers, I thank them for always keeping me humble and down to earth; never letting me become onedimensional in life.

To my wife, Sarah, you are the greatest thing that has ever happened to me and I don't know where I would be without you. You are the most selfless, loving and supportive person I have ever met and I am so blessed that God brought us together to share our lives. You have helped carry the burden of this process without ever complaining and have made it possible for me to get through this. I love you!

Last, and most important, I would like to thank my Savior Jesus Christ. The strength and salvation you have given me through your sacrifice is a debt that can never be paid, nor do you ask us to. The grace you have shown has allowed me to love and pursue you of my own desire and I cannot imagine a life without you. I pray that you would continue to guide me throughout my life and help me to show your love and truth to those around me. 
To my parents who have unwaveringly supported me throughout my entire life and my wife who has selflessly loved and cared for me. 


\section{LIST OF TABLES}

5.1 Structural and superconducting properties $\mathrm{NbN}$ films studied................99

5.2 Relevant deposition conditions and properties of NbTiN films ...............104

6.1 Structural properties and peak deposition power of $\mathrm{Nb} / \mathrm{Cu}$ power series samples done with HiPIMS

6.2 Structural properties and substrate bias of $\mathrm{Nb} / \mathrm{Cu}$ bias series samples done with HiPIMS 


\section{LIST OF FIGURES}

1.1 Theoretical performance enhancement from SIS scheme applied to cavities.

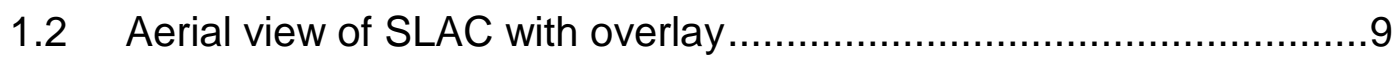

1.3 Simplified diagram of an accelerator ............................................10

1.4 Aerial layout of CERN accelerator facility with overlay ....................11

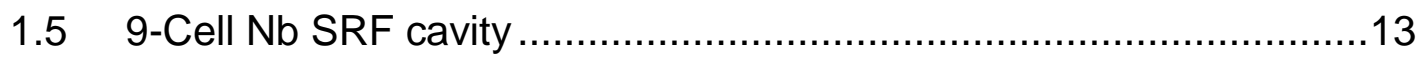

1.6 Simplified diagram of an SRF accelerator cavity setup ...................14

2.1 Simplified plot of resistance vs temperature of normal conducting vs superconducting material

2.2 Simplified plot of heat capacity vs temperature of normal conducting vs superconducting material ...............................................19

2.3 Pictorial representation of the Meissner effect ................................20

2.4 Plots of the magnetization vs applied magnetic field for both Type I and Type II superconductor behavior.

2.5 Simplified pictorial representation of magnetic flux vortex penetration into superconducting material.

2.6 Pictorial representation of BCS superconductivity showing

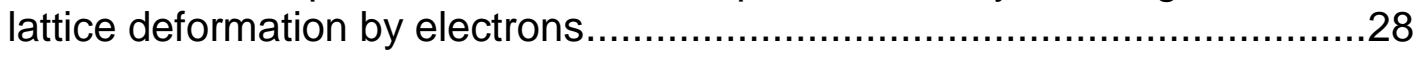

3.1 Depiction of the three growth modes of thin films ............................38

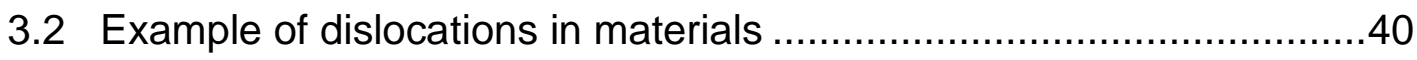

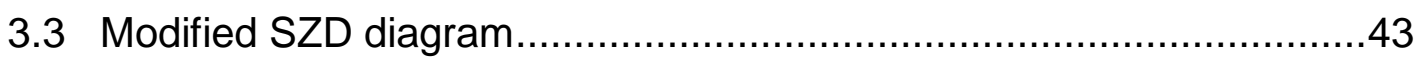

4.1 Simplified diagram of Veeco bell jar ..............................................55

4.2 Schematic of JLab cavity deposition system ...................................56

4.3 Simplified representation of ALD cycles ........................................62

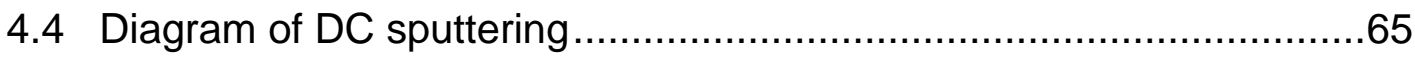

4.5 Schematic of X-ray diffraction from crystal planes ...........................78

4.6 Schematic of a 4-Circle goniometer used for XRD ...........................80 
4.7 Example of EBSD data processed to show crystal

orientation

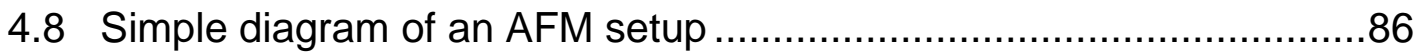

4.9 Example of surface image from AFM data ..........................................

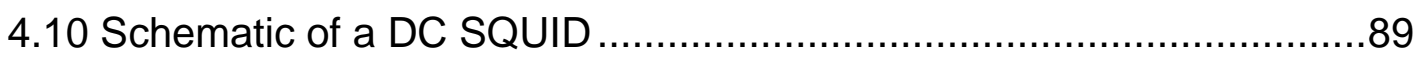

4.11 Example of SQUID sample holder and pickup coils showcasing internal geometry .............................................................

4.12 Example of Tc data collected by SQUID magnetometry ...................92

4.12 Example of $\mathrm{M}$ vs $\mathrm{H}$ data collected by SQUID

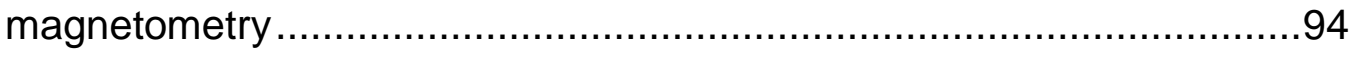

5.1 Plot of the $\mathrm{Hc}_{\mathrm{c}}$ vs thickness for $\mathrm{NbN}$ films showing enhancement with decreasing thickness

5.2 XRD scans of $\mathrm{NbN}$-based multilayer films .......................................102

5.3 SQUID magnetometry data of $\mathrm{M}$ vs $\mathrm{H}$ for $\mathrm{NbN}$-based multilayer films ................................................................................

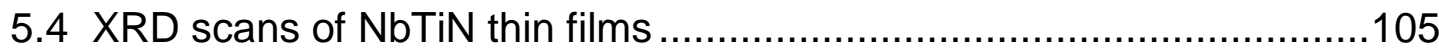

5.5 SQUID M vs H curves for NbTiN thin films .....................................108

5.6 $\mathrm{H}_{\mathrm{C} 1}$ and $\mathrm{T}_{\mathrm{c}}$ vs lattice parameter for NbTiN thin films .........................109

5.7 Plot of the $\mathrm{Hc} 1$ enhancement of $\mathrm{MgB}_{2}$ thin films ............................110

6.1 Representative XRD scan of $\mathrm{Nb}$ films deposited on $\mathrm{Cu}$ substrates via HiPIMS in power series

6.2 EBSD scans of bare Cu substrate, low peak power and high peak power $\mathrm{Nb} / \mathrm{Cu}$ samples

6.3 Representative AFM scan from $\mathrm{Nb} / \mathrm{Cu}$ sample in HiPIMS power series

6.4 Plot of out-of-plane lattice parameter and grain size vs peak power for $\mathrm{Nb} / \mathrm{Cu}$ samples

6.5 Plot of RMS roughness vs peak power for $\mathrm{Nb} / \mathrm{Cu}$ samples

6.6 Plot of out-of-plane lattice parameter and grain size vs applied substrate bias for $\mathrm{Nb} / \mathrm{Cu}$ samples 
6.7 Plot of RMS roughness vs applied substrate bias for

$\mathrm{Nb} / \mathrm{Cu}$ samples

6.8 Example of equator weld crack and trapped chemicals in Cu cavities from manufacturing and processing 123

6.9 Plots of $\mathrm{Q}$ vs $\mathrm{E}$ for $\mathrm{Nb} / \mathrm{Nb} 1.5 \mathrm{GHz}$ cavity coatings.........................124

6.10 Plots of $\mathrm{Q}$ vs $\mathrm{E}$ for $\mathrm{Nb} / \mathrm{Cu} 1.3 \mathrm{GHz}$ cavity coatings showing increased residual resistance due to incomplete coverage. 


\section{CHAPTER 1}

\section{Introduction}

Thin films are an extremely powerful technology with almost limitless ability to create materials with new or altered properties and, as a result, new devices and applications. Simply, thin films are defined as materials where one dimension is highly constrained relative to the other two or a system in which the properties are determined by the surface energy. Thin film technology is widely used throughout many industries and areas of science for everything from semiconductors to tool coatings, detectors and much more. Thin films can be created in a multitude of ways, but these can be grouped into two main categories: physical vapor deposition (PVD) and chemical vapor deposition (CVD) techniques. PVD techniques include methods such as sputtering, electron beam evaporation, laser ablation and cathodic arc, while CVD techniques include atomic layer deposition, plasma-enhanced CVD and hot wall CVD. The two main components of thin films are: the microstructure, that is, the microscopic crystal structure, and the surface morphology. The power of thin films lies in the fact that thin film growth is a highly dynamic process where the kinetics involved during nucleation and growth on the microscopic level are the key determining factors for the resulting structure, morphology and, therefore, properties of the film. [1] [2] [3] [4] 
Thin films are of intense interest due to the fact that by altering conditions or parameters during deposition one can alter certain properties of the resulting films created. Thin films thus offer a cost-effective method of creating engineered surfaces while also opening a rich set of opportunities to engineer material properties for certain applications, especially those that are surface dependent. Once the desired properties of the films have been determined by the intended application, the films may be tailored by careful alteration of the deposition conditions or parameters, such as pressure, energy, temperature and others. In this respect, thin films offer, with the understanding of film growth and using the tools and parameters available, the ability to create materials with properties tailored for use in specific applications.

One possible area for thin film application is that of particle accelerator technology. Specifically, the use of thin films for the enhancement of superconducting radio frequency (SRF) accelerating cavities holds great promise due to the ability to tailor the film properties, especially at the crucial active SRF surface layer, allowing increased control and performance. Current SRF accelerator cavities, typically made of bulk niobium $(\mathrm{Nb})$, suffer from poor thermal conductivity, issues with manufacturing reproducibility and a fundamental upper limit of energy, or accelerating gradient, attainable based upon the specific material properties of Nb. Specifically for SRF, thin films have been proposed for solving many of the current problems and limitations in the field. First, $\mathrm{Nb}$ films can be deposited onto bulk SRF resonant cavities made of more suitable materials that are cheaper and have enhanced properties for accelerator operation. In the past, the material of choice for the bulk $\mathrm{Nb}$ replacement has been $\mathrm{Cu}$. $\mathrm{Cu}$ is relatively inexpensive and 
common compared to $\mathrm{Nb}$, has a much higher thermal conductivity, is easy to machine and is readily adaptable to other parts of accelerator structures. However, $\mathrm{Cu}$ is not the only material possible for this application; other castable metals such as Al are also under consideration. Another advantage of thin film cavities is that, once the desired performance is achieved, the cavity manufacturing is much more reliable and requires fewer processing concerns due to the precise nature of thin films and the dependence on finely-controlled and reproducible deposition parameters.

$\mathrm{Nb}$ thin films on Cu cavities were attempted first by CERN in the 1980's for the LEP-II project [5]. In order to coat the large $352 \mathrm{MHz} \mathrm{Cu}$ cavities for LEP-II, low-energy condensation methods were used in the form of DC sputtering. The resulting cavities were successes in many ways and are still utilized today in accelerators such as the LHC. They were lower cost than the bulk $\mathrm{Nb}$ alternative; had high reproducibility; high efficiency, $\mathrm{Q}$, at low field; and exhibited a lack of sensitivity to the ambient magnetic field present during cooldown, which helped lower cost by requiring less or no magnetic shielding. However, there were a few drawbacks. First, the cavities over the years have shown signs of poor adhesion and even film peeling resulting in the need to replace cavities over time. Also, while the cavities exhibited extremely high low-field $Q$ values, some in the $10^{11}$ range, they could not achieve high accelerating gradients due to a strong negative dependence of the cavity $Q$ on accelerating field. This field dependent $Q$ phenomenon was coined the "Q-slope". Over the years, a multitude of research has been done on trying to find solutions to the Q-slope, all of which has typically made use of low-energy condensation methods [6] [7] [8]. Also, of particular note, there is still no widely agreed-upon cause of 
the observed Q-slope. While there are many proposed causes, such as poor adhesion leading to micro-heating and thermal feedback loop runaway, poor film microstructure and magnetic effects, none up to this point have been conclusive in explaining or solving the Q-slope problem, although not all have been properly explored. [9] [10]
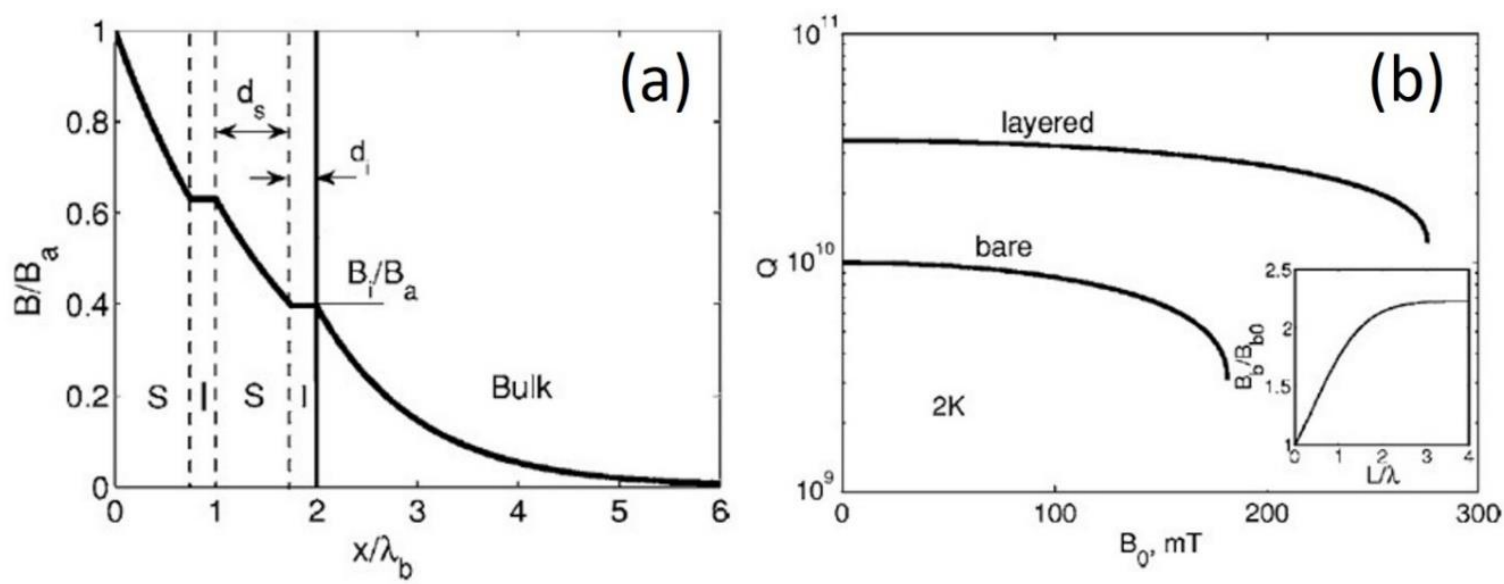

Figure 1.1: (a) Diagram of theoretical stepping down of surface magnetic field through SIS multilayers before reaching bulk cavity; (b) theoretical increase in SRF cavity Q with SIS multilayers applied [11].

Another proposed application of thin films to SRF cavities is to enhance the maximum accelerating gradient, or surface magnetic field, the cavities can achieve before breakdown of superconductivity. A theory proposed by Alex Gurevich [11] puts forth the prospect of coating the inner, active surface of an SRF cavity with alternating superconducting (S) and insulating (I) films. As can be seen in Figure 1.1, this SIS scheme would shield the underlying SRF cavity from the applied magnetic fields, shown in (a), and therefore allow for higher surface magnetic fields to be created while staying in the superconducting state thus increasing the maximum accelerating gradient beyond the bulk $\mathrm{Nb}$ limit of $\sim 50 \mathrm{MV} / \mathrm{m}$, as well as theoretically improving the $\mathrm{Q}$ of the cavities, shown in (b). The theory is based upon the phenomenon observed for type II superconducting 
thin films that when thinner than their London penetration depth, the characteristic length scale over which the magnetic field decays into the material, they exhibit an enhanced lower critical field in the following relationship,

$$
H_{c 1}=\frac{2 \emptyset_{0}}{\pi d^{2}} \ln \frac{d}{\xi} \quad(d<\lambda)
$$

where $d$ is the thickness, $\xi$ is the coherence length, to be discussed later, and $\emptyset_{0}$ is the magnetic fluxon, as long as the applied magnetic field is parallel to the surface, as it is for SRF accelerator cavities operating in the $\mathrm{TM}_{010}$ mode. In order to implement the SIS scheme, particular attention must be made with respect to the film materials to be used. The two main properties necessary for any superconducting material proposed are: a higher Tc and a higher critical field (Bc) than the bulk Nb. As a result, some proposed materials for this scheme are: $\mathrm{NbTiN}, \mathrm{NbN}, \mathrm{MgB}_{2}$ and $\mathrm{Nb}_{3} \mathrm{Sn}$. A more thorough and indepth exploration of this multilayer theory is reserved for the reader through the many available resources [11] [12] [13] [14].

While thin films hold great promise, they are a relatively new technique to the field of SRF and, while they have been employed with limited success in the past, have a long way to go in order to realize their full potential. New energetic condensation techniques offer the promise of being able to create superconducting thin films for SRF application with much improved film quality and resulting performance, since superconductors are extremely sensitive to film quality. Within SRF, Nb thin films currently offer the future ability of being able to create thin film-based cavities with equivalent, or better, performance, and reduced manufacturing and operating costs due 
to the lower material costs, higher reproducibility, reduced processing costs and increased cavity thermal conductivity. Also, the adoption of new superconducting materials and the proposed multilayer SIS scheme offers the ability to create new SRF accelerating cavities, based upon this technology, that are able to reach new levels of cavity efficiency and accelerating gradients beyond the current bulk $\mathrm{Nb}$ limitation. As a result, this showcases a path forward to a future with higher energy and smaller scale particle accelerators.

\subsection{Accelerator History and Fundamentals}

Particle accelerators are widely used around the world as an invaluable tool for many applications. Accelerators are used for everything from scientific research and medical applications to industry and defense. Within the medical field, accelerators are used in treating cancer, creating radioactive marker materials and for fundamental biological studies, while the industrial sector uses smaller particle accelerators for many applications such as: ion implantation, food irradiation and many more. For defense, particle accelerators can be used to create free electron lasers (FELs), like the Thomas Jefferson National Accelerator Facility (JLab) FEL prototype, feasibly capable of shooting down hostile missiles. Perhaps the most large-scale use of particle accelerators is in the scientific realm. High energy particle accelerators have made it possible for the creation of an entire scientific field, that of high energy and nuclear physics [15] [16]. Without highenergy particles created in modern accelerators, like those located at Fermilab, CERN and many others, much of physics would remain out of reach due to the small length scales 
and high energy required for investigation. Accelerators have advanced tremendously over the last 100 years and unlocked realms of physics that were only being dreamt of in the early and mid-1900s. Using accelerators, we have been able to probe the inner structure of matter and even been able to create and observe the Higgs Boson at the Large Hadron Collider (LHC) [17].

With the discovery of superconductivity by Onnes in 1911 and the advent of low temperature cryogenics, and thanks to the increased availability of liquid helium in the 1950s, accelerators have made large advances since the early normal conducting copper (Cu) accelerators. Modern accelerators, utilizing ever-advancing superconducting technology in the form of superconducting radio frequency (SRF) accelerator cavities, have begun to push the material limits of accelerating gradient and energy of $\sim 50 \mathrm{MV} / \mathrm{m}$ for bulk niobium ( $\mathrm{Nb}$ ) while also making continuous-wave operation of particle accelerators economically feasible in stark contrast to normal conducting technology. New and innovative technologies are needed in order to push beyond the current limitations as well as make accelerators more reliable and economical.

Fundamentally, the main purpose of an accelerator is to impart energy to a beam of particles that can be directed and utilized for an array of purposes. There is a wide variety of ways in which this can be accomplished. From the early static electric accelerators, like Van der Graaf generators, which are still in wide use around the world today, accelerators have advanced to ever more complicated and powerful machines capable of probing the subatomic structure of matter. One of the biggest advances in the early history of particle acceleration is the advancement in radio frequency (RF) technology. With access to higher 
power RF sources, accelerators could be created using the principle of resonant structures. This idea for using RF resonances to accelerate charged particles was first proposed by Ising in 1924, but the first prototype was created by Wideröe in 1928 [15].Early on, these resonant structures took the form of cyclotron accelerators. However, the ever-present need for higher energies pushed the sizes of these accelerators and reached their fundamental limit of acceleration where the radiation losses halted higher energy beam production.

The next iteration in route to modern accelerators was that of cylindrically-symmetric $\mathrm{RF}$ resonant cavities made of normal conducting materials such as $\mathrm{Cu}$. Fundamentally, RF resonant cavity structures impart energy to well-timed charged particles that pass through them. This is accomplished by the fact that the cavities typically operate in what is called the TM010 mode where, when at resonant frequency, a longitudinal electric field is created along with a transverse magnetic field circulating around in the cavity, thus the "TM" designation. Since the RF field equally creates an electric field in the desired motion of acceleration and opposite to it, careful timing management is needed so as to ensure that the particles being accelerated see the leading edge of the RF wave and thereby only experience acceleration in the desired direction. This is typically done by using specific dimensions or spacing between accelerator cavities. Interestingly, within a certain range, particle accelerators have self-managing timing by the fact that particles arriving early experience less of an acceleration while those arriving later experience a little bit more thus compressing the bunches of particles together and keeping them in time with the RF wave [15]. 
Modern large-scale accelerators can be grouped into two main designs. The first is that of a linear accelerator or LINAC. Linear accelerators such as the Stanford Linear Accelerator (SLAC), shown in Figure 1.2, or their new Linear Coherent Light Source II (LCLSII), are simply multiple accelerating cavities or structures put together into a long line. The energy of the resulting particle beam is then determined by the accelerating gradient present within each cavity and the number of cavities within the length of the accelerator. For example, the existing normal conducting linear accelerator at SLAC is two miles long and can accelerate electrons or positrons up to $50 \mathrm{GeV}$, while the new LCLS-II accelerator at SLAC will be able to accelerate charged particles, electrons in this case, up to $4 \mathrm{GeV}$ over $500 \mathrm{~m}$ while increasing the beam current by several orders of magnitude [18]. Two major drawbacks to linear accelerators are the relatively low beam current achievable and the need for increased length as the desire for higher energy particles increases, which at higher accelerations results in large downstream radiation cones. These are perhaps the main limiting factors and helped drive the development of synchrotron accelerators to overcome these challenges.

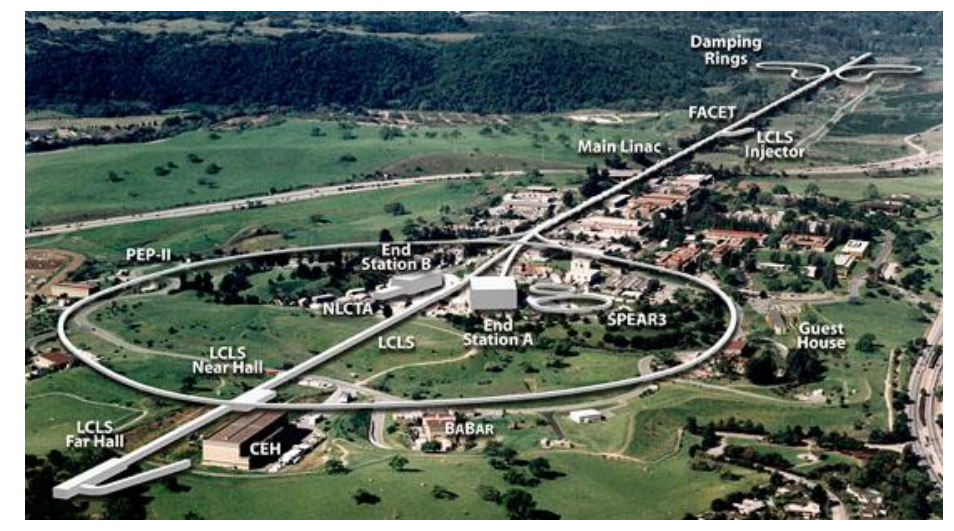

Figure 1.2: Aerial view of the Stanford linear accelerator (SLAC) with overlay. Image from: https://project.Isst.org/safety/SLAC. 
Synchrotron accelerators, the second design, are perhaps the most iconic large-scale particle accelerators today. They are the design for accelerators such as the LHC at CERN and have become a staple in high-energy physics. Synchrotrons still work on the principle of linear acceleration, but, after a bunch of particles has been accelerated through a LINAC section they are deflected and bent around to another LINAC section opposite the first. The layout of synchrotrons closely resembles that of a race track, with long straight sections, the LINACs followed by deflecting superconducting magnets for bending the beam in an ellipse, as shown in Figure 1.3. Using this technique, accelerators can be made that are capable of achieving very high energies, like the $6.5 \mathrm{TeV}$ per beam energy of the LHC, by the process of being able to reuse a LINAC multiple times for acceleration. As particles circulate around the accelerator, they experience acceleration each time they enter the LINAC sections. By this method, the energy can be controlled by the operating gradient of the LINACs and by the number of passes the particles undergo. Some particle accelerators even include storage rings where particles may be maintained for extended periods of time in preparation for supplying higher current to certain experimental areas.

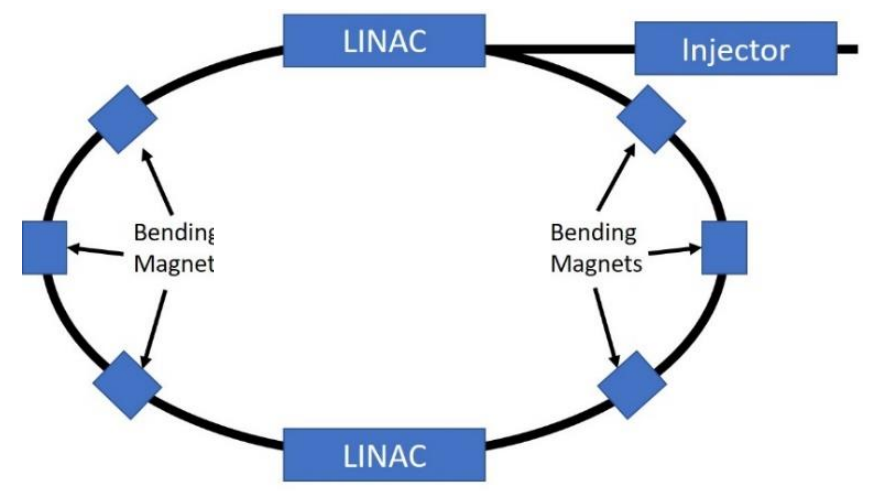

Figure 1.3: Simple diagram of a circular accelerator. 
As can be seen, the synchrotron overcomes the two major disadvantages of the LINAC design. However, synchrotrons have drawbacks of their own. First, they can be enormously expensive to make and can be much larger in terms of overall footprint than linear accelerators, although smaller in size compared to an equivalent energy LINAC. For example, the LHC is $27 \mathrm{~km}$, or 17 miles, in circumference, much larger than SLAC and the scale of which can be seen in Figure 1.4. In large part, this is coupled with the second drawback that, because charged particles emit radiation as they are accelerated, and synchrotrons accelerate particles both linearly and angularly, at higher energies the beam loses a significant portion of its energy per pass via radiation generation. Therefore, efforts are made to increase the radius of the accelerator and therefore decrease the angular acceleration per unit length experienced by the particles. However, this reaches a point of becoming cost prohibitive in size while still not eliminating the energy loss to radiation. [15]

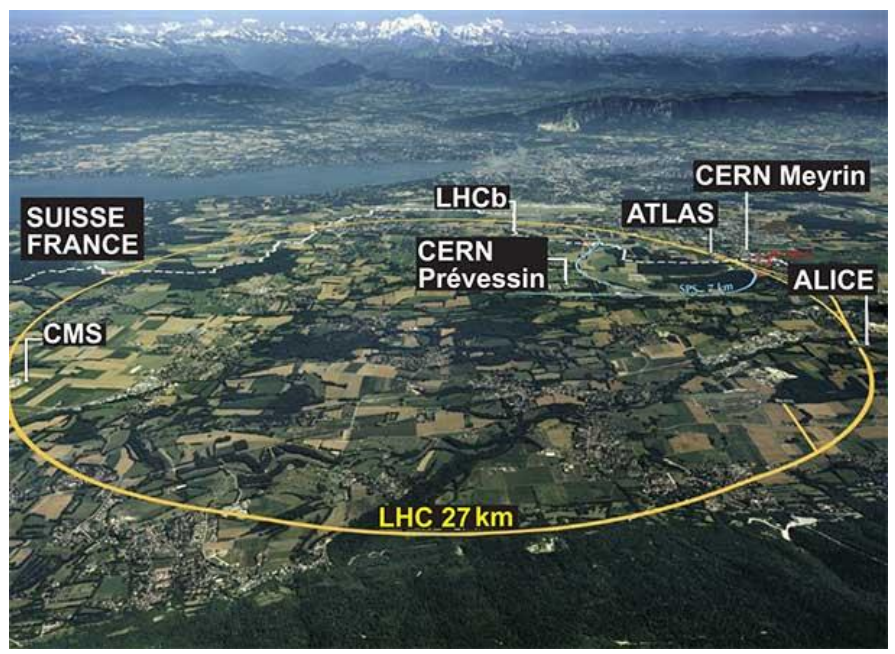

Figure 1.4: Aerial layout with overlay of CERN accelerators. Image by CERN. 
In the early days of particle accelerators, normal conducting RF resonant cavities were used to accelerate charged particles. Such cavities are typically made of $\mathrm{Cu}$ and have some large drawbacks, the two greatest of which are large RF power dissipation in the walls of the cavity and poor transfer of RF power to the beam, which are related and can severely limit accelerator performance. As RF power is increased inside a cavity, RF dissipation, which presents itself as heat, grows quadratically. Due to a plethora of reasons, such as: safety, mechanical stability, vacuum, etc., the heat generated due to the RF losses requires operation to be limited to low energy for CW operation (less than $1 \mathrm{MV} / \mathrm{m}$ ), though high energy pulses can be achieved on very short time scales, or duty factor. Along with the RF dissipation leading to heat generation, it also results in a large amount of the RF power being lost in the walls and not efficiently transferring to the particle beam, leading to a low operating efficiency of the RF power systems and the accelerator. Since particle accelerators need to be cooled via refrigeration systems during operation and RF heat generated increases with power, the cost of refrigeration - and therefore operation - grows dramatically with the beam energy desired. Also, since normal conducting cavities have high shunt impedances, a measure of the losses in a cavity largely dependent on cavity geometry where ideally it is high for acceleration mode to minimize power dissipation, this requires that beam apertures be very large. Together, these limitations result in the inability to economically operate high energy and current particle accelerators needed for much of today's scientific research.

In recent years, many of these problems have been reduced with advances in superconducting materials and corresponding technologies. Superconductivity made it 
possible to create a particle accelerator capable of extremely high center of mass beam energy, like the LHC at CERN, and current, like the CEBAF at JLab in Newport News, VA. Today, most accelerators utilize advances in superconducting technology throughout their operation, from the acceleration of the charged particles via SRF cavities to the bending of the accelerator beam via extremely high field superconducting magnets. While many normal conducting particle accelerators are still in use today and very successful, SRF-based particle accelerators are the most advantageous and economical design for modern high energy and duty cycle applications.

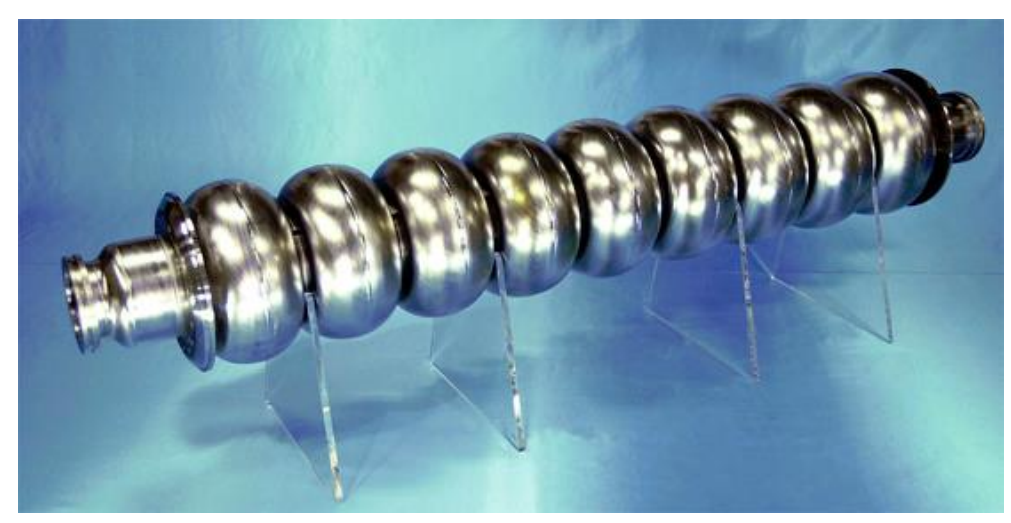

Figure 1.5: 9-Cell Nb SRF accelerator cavity, from JLab.org

SRF accelerator cavities were first proposed by W. Fairbank at Stanford in 1961 [19] and the first SRF electron accelerator was made at Stanford and was composed of lead plated Cu cavities with quality factors, defined as the energy lost per RF cycle of the cavity, around $10^{8}$. Today, the modern SRF accelerator cavities are made of bulk $\mathrm{Nb}$, as it has the highest lower critical field $(\mathrm{Hc} \approx \approx 190 \mathrm{mT})$ and transition temperature $(\mathrm{Tc} \approx 9.2 \mathrm{~K})$ of all the elemental superconductors along and is relatively abundant, as well as easy to refine and machine. A picture of a modern 9-cell Nb SRF cavity is shown in Figure 1.5 while Figure 
1.6 shows a cartoon drawing of a basic SRF cavity setup with a liquid helium cooling bath and RF input probe. The purity and metallurgical properties of the $\mathrm{Nb}$ used for cavities, along with the material processing and manufacturing, plays a key role in the resulting cavity performance and is a large area of interest in SRF R\&D [20] [21] [22] [23]. The main properties of interest for any SRF cavity are its maximum accelerating gradient and quality factor (also as a function of gradient), while the specific shape and type of SRF cavity is determined by the desired application, heavy ion or electron acceleration, kicker cavities and so on, and operating specifications, high or low beta. Over the years, Nb SRF cavities have slowly and consistently improved as a result of improvements in material processing, cavity design and cryogenics, culminating in the current state of the field with modern $\mathrm{Nb}$ SRF cavities pushing the theoretical performance limits for accelerating gradient and exhibiting quality factors in the $10^{11}$ range, the highest ever shown in any natural system. [24]

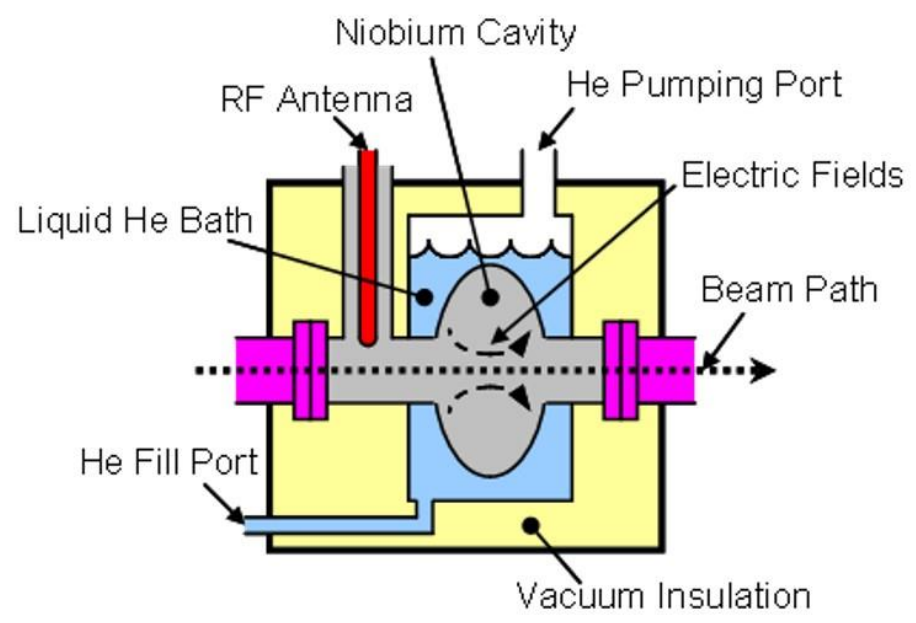

Figure 1.6: Simplified diagram of SRF accelerator cavity setup with RF antenna and liquid He bath capable of cooling to $2 \mathrm{~K}$ via pumping of He bath. 
While bulk Nb cavities have reached unprecedented performance levels, as with any system, there are drawbacks and a desire to push beyond the limitations imposed by the material choice. First, Nb cavities are poor thermal conductors and therefore as heat is generated during operation, the efficiency with which the heat is transported from the inner active surface to the surrounding helium bath is much lower than desired. This leads to high operating costs by requiring more refrigeration to overcome the issue. Second, $\mathrm{Nb}$ cavities, as mentioned earlier, are expensive and very dependent upon the material specifications from vendors. Each material type, for example fine or large grained, is somewhat unique and requires different processing recipes in order to achieve the desired performance levels [20] [21] [22] [23]. The processing techniques involved are typically time-consuming, expensive and involve hazardous chemicals such as hydrofluoric acid (HF). Along with this, the cavities suffer from reproducibility issues and often do not meet performance specifications at first requiring additional rounds of processing which increases cost. The most important drawback to $\mathrm{Nb}$, however, is the fact that $\mathrm{Nb}$ as a superconductor has a maximum surface magnetic field it can sustain before breakdown of superconductivity, to be discussed more in Chapter 2, which limits the maximum accelerating gradient the cavity can achieve.

As demonstrated above, while the accelerator field has advanced greatly by the advent of SRF cavity technology, there are many limitations to SRF accelerating cavities made of bulk $\mathrm{Nb}$, thus making thin film application to this problem appealing for its ability to enhance SRF cavity performance and push the field into the future. To that end, this thesis will focus on experimental structure-property correlations performed on 
superconducting thin films for application in the enhancement of SRF accelerating cavities. The early chapters will provide the reader with a general background to the theoretical topics, introduce key physical properties of importance in interpreting experimental results and give an overview of the experimental hardware and methods used for the creation and characterization of the samples herein. Chapter 5 will discuss results from experiments testing the multilayer theory mentioned above for two proposed materials, $\mathrm{NbN}$ and $\mathrm{NbTiN}$, while Chapter 6 will focus on the results from experimental studies of $\mathrm{Nb}$ films energetically deposited on so-called coupon samples and on SRF cavities. Finally, chapter 7 will conclude the thesis by summarizing the work done and provide an outlook for the future of the field and specific experiments to be performed in the pursuit of further understanding. 


\section{CHAPTER 2}

\section{Superconductivity}

Since the successful creation of liquid helium in 1908 and the resulting discovery of the physical phenomenon of superconductivity in 1911 by Heiki Kamerlingh-Onnes, culminating in his receiving the 1913 Nobel Prize, the field of low-temperature physics and, specifically, superconductivity has been on a fast-paced trajectory. In the past decades, superconductivity has been at the center of intense scientific and industrial research for applications culminating in new technologies that harness strong magnetic fields, such as magnetic resonance imaging (MRI), nuclear magnetic resonance (NMR), plasma confinement, accelerators, extremely sensitive magnetic measurements (such as SQUIDs to be discussed more in Chapter 4) and low loss power generation and transmission [25]. Superconductivity, especially in new, high-temperature materials, has been one of the greatest scientific discoveries in the last 100 years. In this chapter, we will discuss the theoretical background of superconductivity through multiple theories, each with their own strengths and weaknesses, while stressing important properties needed later in the experimental discussion.

In order to understand superconductivity and the theories that help describe it, it is useful to understand that superconductivity was first an experimentally discovered phenomenon with no theoretical predictions beforehand. More so, it would be many 
decades after its discovery before a true microscopic theory would be proposed to describe the observed mechanisms behind superconductivity. In 1911, Onnes discovered superconductivity in $\mathrm{Hg}$, which was manufactured with high purity at the time. The first experimental observation of the phenomenon was the sharp disappearance of DC electrical resistivity at a critical temperature $(4.3 \mathrm{~K}$ for $\mathrm{Hg})$, which is in stark contrast to the behavior of normal metals, as can be seen in Figure 2.1, and to one proposed theory at the time which predicted a rise in the resistance to infinity as the temperature was lowered toward absolute zero or to the behavior of non-superconducting materials. While we cannot say for certain that there is absolutely no resistance, to this day it is below measurable experimental sensitivity and has only been able to have upper limits placed upon it of $\sim 10^{-12}$, truly a "super" conductor [25]. After this discovery, a race took place to find more such materials and understand more about their properties.

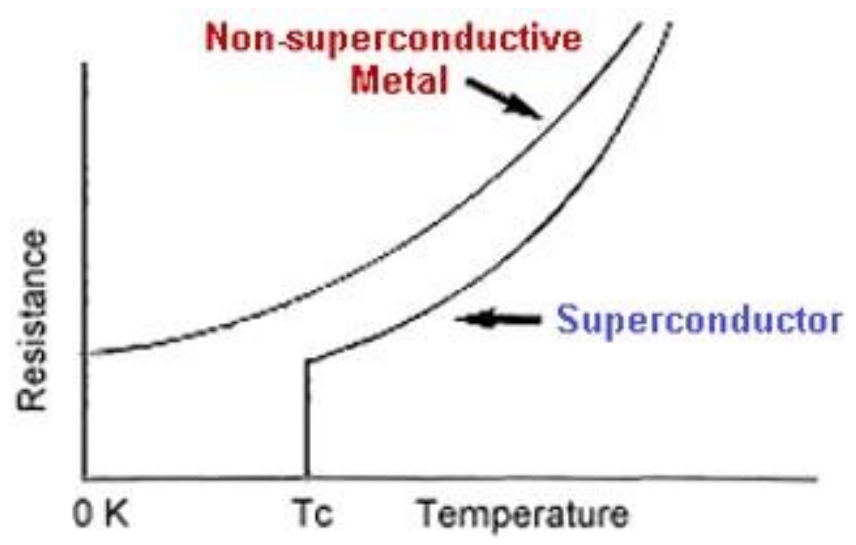

Figure 2.1: Representation of the difference in behavior of the resistance vs temperature of normal conductors compared to superconductors. Image from: cern.ch

The second experimental observation was the isotope effect, in which particular isotopes of a superconducting material can have different transition temperatures based 
upon their nuclear mass. This hinted at the correlation of the structure or lattice of the material with the phenomenon and had significant impact in theoretical formulations [25] [26]. Third, the heat capacity of superconductors is very unique. While they follow normal material behavior decreasing linearly down to their transition temperature, at the transition temperature, Tc, they experience a sharp jump up to some larger value and then decreases exponentially with decreasing temperature, as shown in Figure 2.2. Fourth, the electrical resistance is not zero in all circumstances. Under RF conditions, there exists some critical frequency for each material below which the resistance is unmeasurable, but above it resistance appears. This was first observed optically and was attributed to the optical superconducting energy gap and implies electronic order within the material. [27]

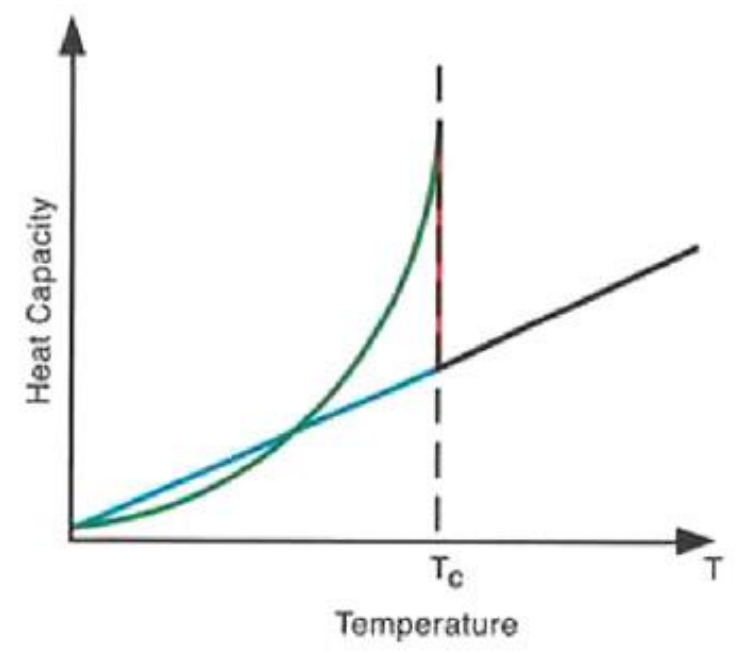

Figure 2.2: Graph of the heat capacity vs temperature of a superconductor compared to a normal metal. From Superconductivity, Revised Edition by V. L. Ginzburg and E. A. Andryushin and published by World Scientific. 


\subsection{Meissner Effect and Magnetic Behavior}

The magnetic behavior of superconductors is, besides the zero DC resistance below Tc, perhaps the most unique property. In 1933, Meissner and Ochsenfeld discovered the peculiar behavior of superconductors to spontaneously expel all magnetic flux from the bulk material when transitioning into superconducting state [27] [25]. This phenomenon, pictorially shown in Figure 2.3, was named the Meissner effect. This perfect diamagnetism has since become a defining trait of all superconductors. Since the Meissner effect is so important to the description of superconductors and for the research done within this thesis, it is worthwhile to take time to have a more detailed discussion of it.
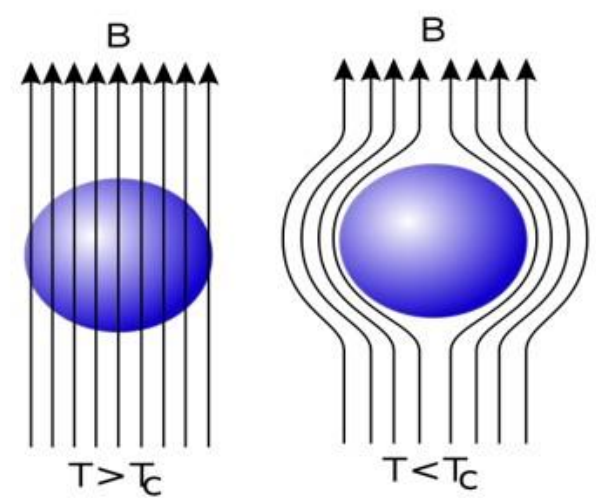

Figure 2.3: Pictorial representation of the Meissner effect present in superconductors. Image from: https://en.wikipedia.org/wiki/Meissner_effect. Credit:Piotr Jaworski.

Conceptually, the Meissner effect is understood as a result of the fact that superconducting materials in the presence of an applied magnetic field respond by creating super-currents that induce a B field equal and opposite to the applied field. Also, 
if the material is exposed to an applied field in the normal state and then transitioned into the superconducting state, at the critical temperature it will spontaneously expel all flux from within the material. However, it is important to note that this is true up to some material dependent critical field value, which will be discussed in more detail shortly. The Meissner effect cannot be fully explained using classical electrodynamics and therefore required new theories or modifications to existing models.

The magnetic properties of superconductors play a key role in their description and classification. There are many ways to categorize superconductors, such as: material (elemental, alloy, ceramic, etc.) and relation to BCS theory (conventional vs unconventional). However, one of the most common classification methods has to do with the material's behavior in the presence of an applied magnetic field. A figure representing the two possible responses is shown in Figure 2.4. The first behavior in Figure 2.4(a) corresponds to materials that remain in the Meissner state up to a single critical field value above which the material abruptly exits the superconducting state and remains in the normal state even if below the critical temperature. The superconductors that exhibit this behavior are referred to as Type-I superconductors.

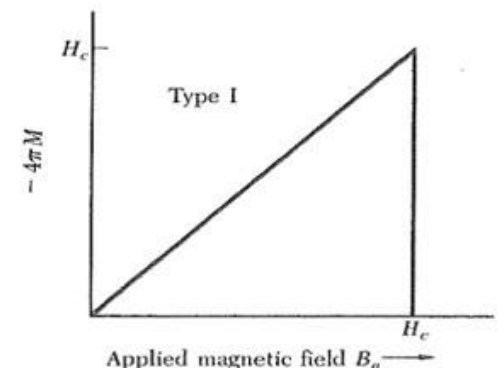

(a)

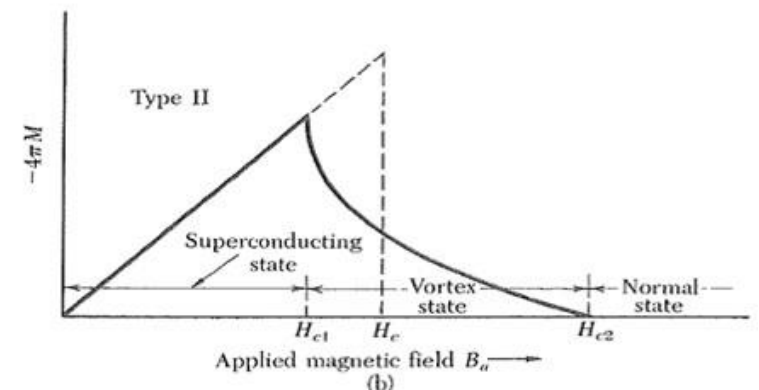

(b)

Figure 2.4: Graph of the magnetization of superconductors plotted against applied magnetic field. (a) Behavior representative of Type I and (b) of Type II superconductors. Image from: Image: "Solid State Physics" Kittel. 
The second behavior shown in Figure $2.4(\mathrm{~b})$ is characterized by the presence of two critical field values. First, above the lower critical field $\left(H_{c 1}\right)$ value the material enters a mixed state, rather than completely leaving the superconducting state like Type-I superconductors, wherein magnetic fields penetrate the bulk material as quantized vortices with normal conducting cores, as shown in Figure 2.5. The material responds to these vortices by creating super currents that surround the vortex, containing the magnetic fields within this small region, and allowing the superconductor to still flow DC current without resistance; the case is much more complicated in the RF mode, which will be discussed later. As the magnetic field is increased, more vortices penetrate the material, consuming more of the material volume until an upper critical field $\left(H_{c 2}\right)$ value is reached, at which point the vortex cores begin to overlap forcing the material to exit the superconducting state. These materials are referred to as Type-II superconductors and, due to the mixed state between $\mathrm{H}_{\mathrm{C} 1}$ and $\mathrm{H}_{\mathrm{C} 2}$ being able to exist up to very high magnetic field values, have proven to be incredibly useful for high magnetic field applications.

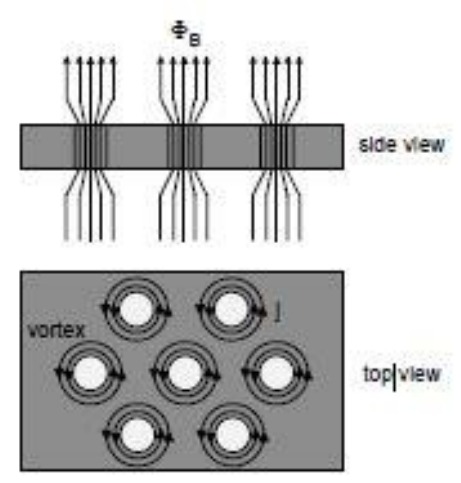

Figure 2.5: Simplified representation of vortex penetration into Type II superconductors in mixed state. Image credit: Philip Hofmann. 
With the experimental evidence in mind, many attempts have been made since the discovery of superconductivity to formulate a theoretical explanation. However, this has proven one of the most difficult problems in theoretical physics and even some of the greatest minds of physics, such as Einstein, worked on the problem with no success. Over the years, multiple theories have been created that help explain certain aspects of superconductivity, although most focused on explaining macroscopic properties of superconductors. It wouldn't be until 1957, 46 years after the discovery of superconductivity, that a true microscopic theory would be put forth. Some of the key theories still used today are the: London theory, the Ginzburg-Landau theory and the BCS theory. In the following sections, we will discuss each theory's fundamental concepts, strengths and weaknesses.

\subsection{London Theory}

The first theory to effectively explain some of the macroscopic behavior of superconductors was the London theory. Created by brothers Fritz and Heinz London in the 1930's, the London theory, and its key London equations, is an early phenomenological description of the electrodynamic behavior of superconductors. Specifically, the two key aspects were the explanation of the DC resistivity and the Meissner effect. The London theory begins by assuming the superconducting charge carriers are charged bosons and experience uniform Lorentz forces arising from external E and $\mathbf{B}$ fields. Using the following equation for the current density of $\mathrm{N}$ electrons: 


$$
\boldsymbol{j}(\boldsymbol{r})=\sum_{\ell=1}^{N} \int\left\{\frac{e \hbar}{2 m i}\left[\psi^{*} \nabla \ell \psi-\psi \nabla \ell \psi^{*}\right]-\frac{e^{2}}{m c} \boldsymbol{A}\left(\boldsymbol{r}_{\ell}\right) \psi^{*} \psi\right\} \times \delta\left(\boldsymbol{r}-\boldsymbol{r}_{\ell}\right) d \boldsymbol{r}_{1}, \ldots, d \boldsymbol{r}_{N}
$$

where $\psi\left(\boldsymbol{r}_{1}, \ldots, \boldsymbol{r}_{N}\right)$ is the many-electron wavefunction, the first term represents a paramagnetic term and the second a diamagnetic term, and the assumption that the wavefunction is rigid with respect to the transverse vector potential $\mathbf{A}$, such that $\nabla \cdot \mathbf{A}=$ 0 , the brothers were able to show that the paramagnetic term vanishes and write the equation as follows.

$$
\boldsymbol{j}(\boldsymbol{r})=-\frac{e^{2}}{m c} n_{s} \boldsymbol{A}
$$

where $n_{s}$ is the density of superconducting charges, $e$ is the charge of the electron and $m$ is the electron mass. This is known as the London equation. If one expands this equation in terms of measured fields it yields the following:

$$
\frac{\partial \boldsymbol{j}_{s}}{\partial t}=\frac{n_{s} e^{2}}{m} \boldsymbol{E}
$$

and

$$
\nabla \times \boldsymbol{j}_{s}+\frac{n_{s} e^{2}}{m} \boldsymbol{B}=0
$$

The first equation represents the infinite conductivity, while the second connects the applied magnetic field with the current density and contains the necessary leap to explain the Meissner effect. Then applying Ampere's law,

$$
\nabla \times \boldsymbol{B}=\mu_{o} \boldsymbol{j}_{S}
$$


one can attain the following expression for the magnetic field inside a superconductor

$$
\nabla^{2} \boldsymbol{B}=\frac{1}{\lambda_{L}^{2}} \boldsymbol{B}
$$

where lambda is the termed the London penetration depth. The superconducting current density can similarly be obtained as the following expression:

$$
\nabla^{2} \boldsymbol{j}_{s}=\frac{1}{\lambda_{L}^{2}} \boldsymbol{j}_{s}
$$

To understand the physical significance of the London penetration depth it is useful to look at an example. Consider a simple planar superconductor surface boundary with a vacuum such that the surface is normal to the $x$-axis and the superconductor extends into the positive $x$-direction. If a magnetic field is applied pointing into the $z$ direction, then the corresponding field and charge density within the superconductor can be expressed as:

$$
B_{z}=B_{o} e^{-\frac{x}{\lambda_{L}}} \quad, \quad j_{y}=-\frac{1}{\lambda_{L}} B_{o} e^{-\frac{x}{\lambda_{L}}}
$$

From these equations one can see that the London penetration depth is the characteristic length scale over which the magnetic field exponentially decays in the bulk superconductor showcasing the imperfect shielding of the supercurrents. Also, it shows that the supercurrent is confined to the topmost layers of the superconductor [24] [26] [27]. 


\subsection{Ginzburg-Landau Theory}

The next major theory to help explain superconductivity was developed by Ginzburg and Landau in 1950 as an extension to the above London theory. The theory was based on second-order phase transition theory developed by Landau and desired to take into account the spatial dependence of the superconducting electron density [25] [27]. The Ginzburg-Landau theory has proven to be very useful in explaining the behavior of superconductors, especially Type-II, near $\mathrm{T}_{\mathrm{C}}$, in high magnetic fields and for spatially inhomogeneous systems, such as proximity systems and thin films. The Ginzburg-Landau theory, while complicated (its derivation beyond the scope of this thesis), resulted in two equations that contained the two characteristic lengths for superconductors: namely the penetration depth, $\lambda$, discussed above in the London theory, and the coherence length, $\xi$. While the penetration depth concerns itself with the decay of the magnetic field within the surface of a superconductor, the coherence length, in the Ginzburg-Landau theory, represents the variation in density of the superconducting electrons. It is important to note that the Ginzburg-Landau equations were eventually re-derived from the later microscopic BCS theory.

Finally, another development of the Ginzburg-Landau theory was the institution of a dimensionless parameter, the Ginzburg-Landau parameter. The Ginzburg-Landau parameter

$$
\kappa=\frac{\lambda}{\xi}
$$


is useful in categorizing the magnetic behavior in superconductors. As discussed earlier, there are two types of superconductors: Type-I and Type-II. We can predict the magnetic behavior, and therefore classification, of a superconductor by looking at their parameter as follows:

$$
\kappa<\sqrt{2} \text { [Type-I] } \quad \& \quad \kappa<\frac{1}{\sqrt{2}} \text { [Type-II] }
$$

\subsection{BCS Theory}

It wasn't until 1957 that a quantum mechanical explanation of superconductivity and justification of the London and Ginzburg-Landau equations was developed by Bardeen, Cooper and Schrieffer. The resulting BCS theory is still the most widely used and successful theory of superconductivity and, while not successful for all superconductors, has been expanded to encompass many such materials. The BCS theory rests on the idea of the formation of electrons into bosonic quasiparticles called Cooper pairs. Cooper showed that, for two electrons in the Fermi sea of a material, any attractive potential between the two would necessarily result in a pairing of electrons with opposite spin and momentum. Building upon this idea, BCS theory was developed with the assumption that the mechanism of attraction was the phonon-mediated lattice-electron interaction [26] [27]. 


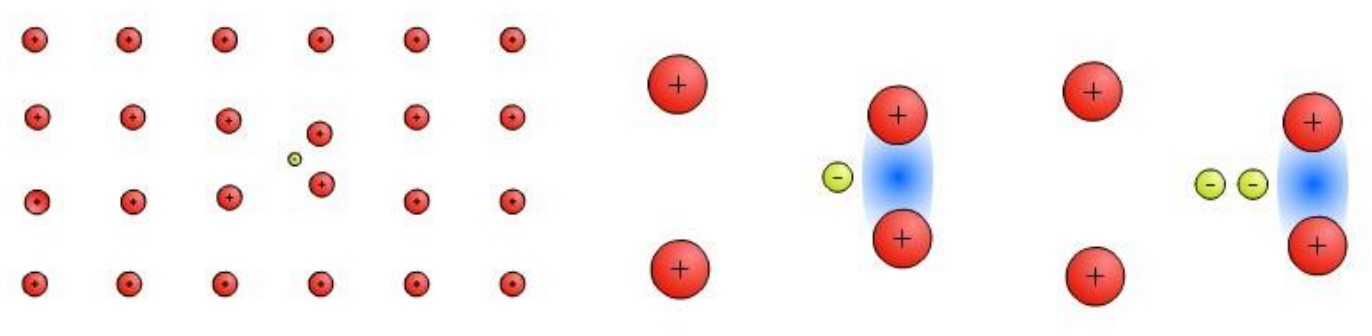

Figure 2.6: Representation of the BCS theory for superconductivity showcasing how electrons displace lattice ions causing attraction with other electrons, thus forming cooper pairs. Image: doitpoms.ac.uk.

Conceptually, one can visualize BCS theory by, shown in Figure 2.6, an electron traveling through the crystal lattice of a superconductor, attracting the positive ions, thus deforming the lattice. This deformation, slow compared to the electron speed, exhibits a net positive potential area that attracts another electron. Through this behavior, two electrons can become correlated within the material and thus form a Cooper pair. In certain materials, as the temperature becomes small the thermal motion is lowered enough for this process to dominate and for the attraction to overcome Coulombic repulsion. Already, we can see that the BCS theory is supported by the isotope effect discussed earlier, as the interaction is fundamentally dependent on the lattice interaction with electrons mediated via phonons. As bosons, the Cooper pairs can all occupy a single quantum state, therefore creating a gap in between the newly-excited electron state and the ground state Fermi energy. This temperature-dependent energy difference - more electrons pair off as the temperature is lowered - is referred to as the energy gap, $\Delta$. BCS theory then relates the energy gap to the $\mathrm{T}_{\mathrm{c}}$ of the material by

$$
1.76 k_{B} T_{C}=\Delta(0)
$$


One aspect of superconductivity and the electron pairing picture that has major implications for SRF cavities and thin films is the thermal conductivity behavior of superconductors. As SRF cavities are in operation there is heat generated in the cavity walls from the surface resistance of the cavity. This heat must be transported from the inner cavity wall and into the cooling reservoir to maintain the cavity temperature and performance level. For most metals in a normal conducting state, the thermal conductivity increases with decreasing temperature. This is because the main mechanism for thermal transport are the valence or conduction, electrons and as the temperature is lowered the electron-phonon scattering is lowered, thus allowing for a larger mean-freepath for the electrons and therefore higher conductivity. However, BCS theory shows that for superconductors, as the temperature is lowered below $T_{c}$, more electrons are condensed into Cooper pairs. While the phonon scattering is still lowered, the lower number of electrons plays a much larger role and the result is an exponentially-decreasing thermal conductivity of most materials in a superconducting state with decreasing temperature, which has been shown experimentally. As an aside, the thermal conductivity also shows interesting behavior in the lattice portion.

While the thermal conductivity is dominated by the electron contribution discussed above, it is possible to extract the lattice portion from the data and compare it with models. The lattice contribution shows interesting behavior with intuitive physical explanations. As the temperature is decreased, there are less phonons in the system, which should lower the thermal conductivity of the lattice. However, at the same time the number of phonons decreases, their mean free path increases due to exponentially 
fewer electrons to scatter from. Indeed, the increase in mean free path has a stronger effect than the lower number of phonons, resulting in an increase in the lattice thermal conductivity. This continues until the temperature is lowered to a point at which the phonon mean free path becomes so large that the phonon scattering from impurities, defects and crystal boundaries begins to dominate and then the thermal conductivity begins to decrease [25].

\subsection{Surface Resistance of Superconductors}

While superconductors exhibit zero DC resistivity, in the presence of a timevarying magnetic field, above a critical frequency they do exhibit losses, although still much lower than those in a normal metal. These losses are referred to as "surface" resistance. An intuitive model that helps understand this phenomenon was proposed by Gorter and Casimir in 1934. The Two-Fluid Model describes the superconducting state as a sea composed of two liquids: "normal" and "superconducting." Within this model, the normal conducting sea is treated as typical normal conducting metal electrons, while the superconducting component is responsible for the zero DC resistivity and the other interesting superconductor behavior. Conceptually, the model describes the superconductors surface resistance by considering a time-varying magnetic field incident upon the surface. Because the superconducting sea elements- i.e. Cooper pairs - have inertia, they do not react instantaneously to the varying field and therefore do not perfectly screen the magnetic field, as can be shown by the existence of the London penetration depth. As a result, the time-varying magnetic field within the surface of the 
material induces an internally-varying electric field, which forces motion within the superconducting and normal electrons. While the superconducting electrons then move without resistance, the normal electrons move and scatter, creating dissipation that results in the observed surface resistance. [24]

More precisely, the time-varying internal electric field causes the superconducting electron pairs to experience an acceleration and current within the material with no dissipation as

$$
J_{s}=-i \frac{n_{c} 2 e^{2}}{m \omega} E_{o} e^{i \omega t}
$$

where $\mathrm{n}_{\mathrm{c}}$ is the density of cooper pairs, $\mathrm{e}$ is the electron charge, $\mathrm{m}$ is the mass of the pair and $\omega$ is the frequency. However, the normal conducting electrons create a current obeying Ohm's law as

$$
J_{n}=\sigma E_{o} e^{i \omega t}
$$

where $\sigma$ is the normal state conductivity. We can then add both fluids' current together, yielding the total current density

$$
J=J_{n}+J_{s}=\sigma E_{o} e^{i \omega t}
$$

where the conductivity is a complex value such that

$$
\sigma=\sigma_{n}-i \sigma_{s}, \quad \text { where } \quad \sigma_{s}=\frac{n_{c} 2 e^{2}}{m \omega}=\frac{1}{\mu_{o} \lambda_{L}^{2} \omega}
$$


As a result, we can express the observed superconductor surface resistance as the real part of the complex impedance

$$
R_{s u r f}=\operatorname{Re}\left(\frac{1}{\lambda_{L}\left(\sigma_{n}-i \sigma_{s}\right)}\right)=\frac{1}{\lambda_{L}} \frac{\sigma_{n}}{\sigma_{n}^{2}+\sigma_{S}^{2}}
$$

Making a small logical assumption that $\sigma_{\mathrm{n}}{ }^{2}<<\sigma_{\mathrm{s}}{ }^{2}$, we can then express the above equation as approximately $R_{\text {surf }} \propto \sigma_{n} /\left(\lambda \sigma_{s}^{2}\right)$, which shows the surface resistance is directly proportional to the normal state conductivity of the material. This is why in the multilayer theory, discussed in the introduction, the materials with higher normal state conductivities such as $\mathrm{NbTiN}$ are more promising candidates.

If we next apply the Drude expression for conductivity, we can retrieve the expression for the surface resistance in BCS theory:

$$
R_{B C S} \propto \lambda_{L}^{3} \omega^{2} l e^{\left(-1.76^{T_{C}} / T\right)}
$$

This shows the dependence on the square of the driving frequency, $\omega$, the mean free path, I, and the exponential dependence on temperature.

\subsection{Residual Resistance}

While the surface resistance is predominantly made up of the BCS surface resistance, $R_{B C S}$, experimentally it is observed that the total surface resistance is always greater than the $\mathrm{R}_{\mathrm{BCS}}$ value. The extraneous, temperature-independent losses that comprise this component are collectively referred to as the "residual resistance", $R_{o}$, such that the total surface resistance, $R_{s}$, of a superconductor is expressed as [24] 


$$
R_{S}=R_{B C S}(T)+R_{o}
$$

Some typical examples of mechanisms that add to the residual losses of a superconductor, especially in SRF cavities, are chemical residues after surface treatments, defects, non-superconducting contaminants in the active surface and trapped magnetic flux, among others [24]. Although such residual losses are typically small, they can strongly affect thin film cavities, as they technically include the field-dependent losses, or Q-slope, observed in $\mathrm{Nb} / \mathrm{Cu}$ cavities and poorly prepared bulk Nb cavities.

An important note for the operation of SRF accelerator cavities is that, not only is the surface resistance non-zero although very small in the $\mathrm{n} \Omega$ range, thus requiring refrigeration to maintain operation temperature, but there also exists trapped magnetic flux in the form of vortices within the material. While theoretically a perfect superconductor expels all magnetic flux, in reality, materials have defects throughout that allow for the penetration and trapping of magnetic flux resistant to the Meissner effect. These defects are referred to as pinning sites and have much to do with the reported quality of RF performance. Pinning sites can be both beneficial and detrimental. For example, a worst-case scenario for SRF cavity operation is the presence of a free magnetic vortex that, under the influence of the varying fields, moves along the surface of the cavity and creates RF losses, thus lowering the cavity performance. A pinning site may be beneficial in this case by trapping the magnetic vortex strongly enough that it locks it in place, not allowing it to move and create losses. However, pinning sites may be detrimental by trapping vortices just enough to resist the Meissner expulsion during 
cooldown but not strongly enough to resist high-field operation thus allowing the vortices to break away at sufficient fields, creating dissipation in the surface and possibly limiting the performance of the cavity. For this reason, magnetic shielding, pinning and flux expulsion are topics of utmost importance for modern SRF accelerator design and operation [24]. 


\section{CHAPTER 3}

\section{Thin Films}

In this chapter, an overview of thin films will be presented to give the reader insight into the physics of thin film growth and the properties that can be tailored by proper control. There are many definitions that help describe thin films such as a system in which one spatial dimension is highly constrained with respect to the others (for example the thickness being much less than the surface area) or, perhaps more specifically, a system in which the material properties are predominantly determined by the surface energy rather than the bulk [1] [2], where the latter definition arises from the fact that surface atoms in a material have increased energy due to the lower number of bonds with surrounding atoms. Thin film growth is a highly dynamic process where the kinetics involved on the microscopic level are the key determining factors for the resulting structure and morphology, and therefore properties, of the film. The two main components of thin films are: the microstructure, that is, microscopic crystal structure, and the surface morphology. The properties desired of thin films are determined by the application and requirements therein. In this respect, thin films offer, with the understanding of film growth and using the tools and parameters available, the ability to create materials with properties tailored for use in specific applications. 


\subsection{Film Growth}

To begin, it is useful to understand the basic steps of creating th in films via physical deposition methods, e.g. evaporation, sputter-deposition, etc. There are four primary stages of film growth: condensation, nucleation, coalescence and growth and thickening [1]. Condensation is the first step of any film growth by physical deposition and, in the case of deposition used in this research, involves the transfer of atoms from a target into the vacuum as a vapor and then onto a substrate surface. Arriving atoms impinging on the substrate surface at some rate can undergo multiple statistical outcomes - they can immediately re-evaporate or reflect from the surface, which removes them from making the film, or they can stick, or adsorb, to the surface. It is these atoms that stick to the surface that will eventually constitute the bulk of the resulting film. When the atoms adsorb onto the surface called adatoms, there are two possibilities for how they will bond with the substrate: chemisorption and physisorption. Chemisorption occurs when the interaction between the adatoms and the substrate is chemical in nature and they bond either by ionic or covalent bonds, losing some of their own identity in the outcome. This form of bonding, while somewhat rare, leads to strong bonds between the first layers of the forming film and the substrate resulting in well adhered films. Physisorption, on the other hand, is described by adatoms that when interacting with the substrate are bonded by the weak Van der Waals force and maintain their individual identity [1].

Once atoms have interacted and "stuck" to the desired substrate surface, they can undergo desorption, that is, re-evaporate or leave the surface, they can diffuse, or, in 
some circumstances, they can remain in place. Diffusion is a very important mechanism in the kinetics of film growth. Atoms adsorbing onto a surface can undergo diffusion into the substrate or diffuse along the surface. The diffusion along the surface, or surface mobility, is a key parameter in film growth as, if atoms are highly mobile within the surface, they are able to encounter nucleation sites, fill vacancies within the newly forming lattice and add to existing growth structures. The desire in many film deposition methods is to increase the energy of deposited atoms in order to increase the surface mobility, thus allowing for dense, well-formed crystals to be created [1].

The second step of film deposition is nucleation. As atoms stick to the substrate and move along the surface, they encounter nucleation sites, typically defects in the substrate surface, and begin to form structures or nuclei. Eventually, the nuclei will reach critical sizes in which they can break apart or will cover enough of the surface that the rate of nuclei creation will be limited and require the merging of nuclei and the beginning of a new surface. This then leads into the third step in film growth, coalescence and growth. Coalescence is the process by which the smaller constituent structures that form from nuclei begin to combine and create the bulk film. In general, there are three modes of film growth during the nucleation and coalescence stages, depicted in Figure 3.1. First, the atoms may begin to grow in a layer-by-layer fashion known as the Frank-van der Merve mode. In the layer mode, the adatom's interaction with the substrate is greater than for their neighbors resulting in increased surface mobility and the creation of a complete monolayer before the start of another. These layers continue to build upon each other until a film has been created. 


\section{a)}

Frank van der Merwe layer-by-layer growth
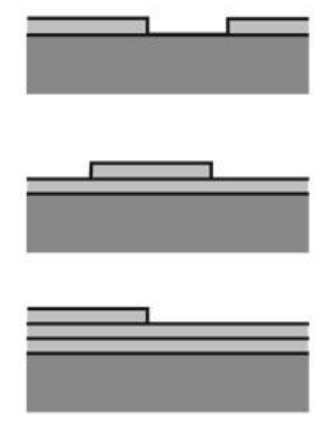

b)

Stranski-Krastanov layerplus-island growth
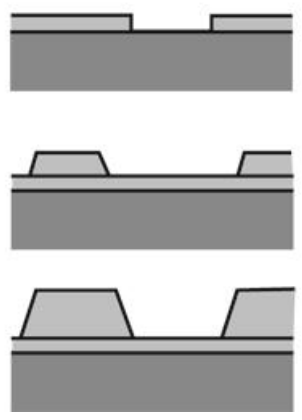

c)

Wolmer-Weber island growth
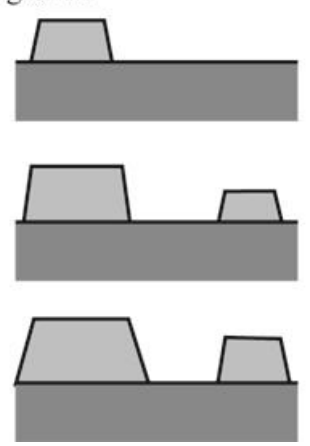

Figure 3.1: Conceptual depiction of the three thin film growth modes: (a) Layer-by-Layer, (b) Layerplus-Island and (c) Island Growth. Image: https://dic.academic.ru.

Second, the film can undergo island growth known as Volmer-Weber growth. In this growth mode, the adatoms attraction to each other is stronger than the interaction with the substrate, resulting in large $3 D$ islands being created on the surface that eventually combine to create the overall film structure. Finally, films may grow in an intermediate, layer-plus-island, mode known as the Stranski-Krastanov mode. Typically, this mode involves the early stages of film growth being layer-by-layer, but as the film thickens the mode switches into the more energetically favorable island growth. Some possible reasons for this transition in growth modes could lie in the lattice mismatchinduced strain at the interface not being able to be continued into the bulk of the film or a symmetry- or orientation-driven force. [1]

The last step of film growth is the thickening of the film. Once the process has begun through the crucial first mono-layers, the process continues for a controlled amount of time determined by the desired film thickness. While thickening may seem a trivial step of the process, it is during this crucial time that the bulk of the film is being 
developed: defects are being created or propagated, contaminants are being brought into the material, grains are being developed and, most importantly, the final film microstructure and surface are being defined.

One of the more important aspects of film growth is the effect of the substrate on the resulting film. The substrate plays a pivotal and influential role in film growth, especially in the initial stages. Early on, the substrate provides a template for the future film lattice and offers nucleation sites in the form of its imperfections like step edges and ledges. Along with the obvious contributions of the substrate to the growth modes, the resulting film's structure may be inherently linked to that of the substrate. Films are typically classified into two categories based upon their relation to the substrate structure, the two extremes of which are epitaxial and amorphous. Epitaxial films, of utmost importance in the research done here, are the result of a strong relationship between the substrate and the film crystallographic orientation. Epitaxy may be broken up into two variations, namely, homoepitaxy and heteroepitaxy. Homoepitaxy is when the film is deposited onto a substrate of the same material. This results in their crystallographic orientation being the same (e.g. $\mathrm{Nb}$ film grown onto a $\mathrm{Nb}$ substrate). Heteroepitaxy, on the other hand, is when materials are grown onto a substrate that has similar lattice structure, and there exists a specific registry of resulting film structure to the substrate crystal structure, for example a $\mathrm{NbN}(002)$ layer being grown on a $\mathrm{MgO}(001)$ substrate, which was done in this research. In some other cases, the substrate lattice and the film lattice are not necessarily in the same out-of-plane orientation. For example, $\mathrm{Nb}$ has a heteroepitaxial relationship with $\mathrm{Cu}$, resulting in a specific $\mathrm{Nb}$ crystallographic orientation 
being possible on a specific $\mathrm{Cu}$ orientation; however they are not the same orientations in each case, such as, $\mathrm{Nb}[011]$ being grown on $\mathrm{Cu}[001]$ [28].

Epitaxial deposition enables fabrication of single-crystal films with well-known and controllable properties for investigation. On the other extreme, the other type of film that can be created are amorphous films. Amorphous films, while rare, are indicative of a lack of long-range order throughout the film and no known correlation between substrate and film crystallography. Typically, it is very difficult to create truly amorphous films, if there is such a thing, and usually involves the cooling of substrates to extremely low temperatures thereby lowering adatom surface mobility and preventing the creation of larger-scale growth structures. In between these two categories, there are polycrystalline films, with varying grain sizes [1].

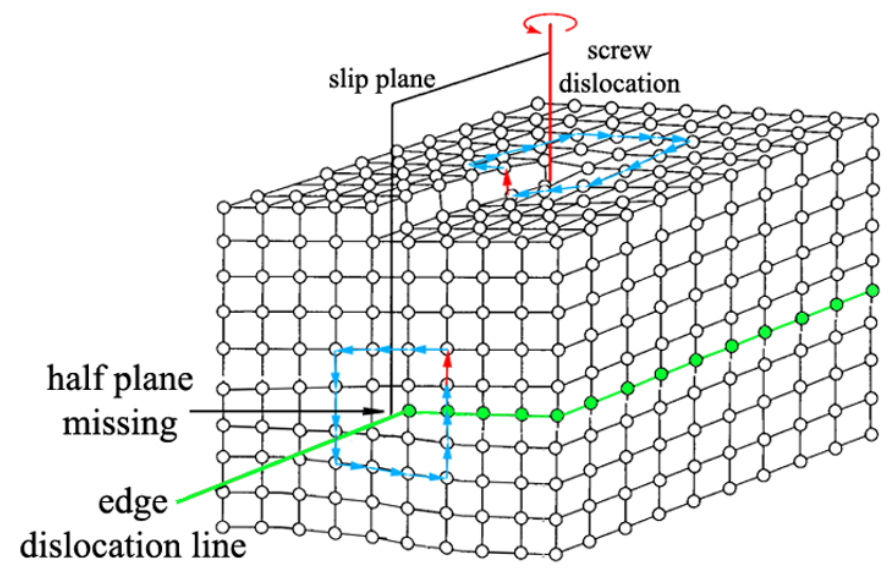

Figure 3.2: Example of dislocations in material lattice showing two types: edge and screw dislocations. Image from: Basics of Molecular Beam Epitaxy (MBE) technique by Lorenzo Morresi, published by Bentham Science Publishers. 
The most important outcome of thin film growth is the resulting film properties. Some typical properties of interest for films after growth are defect density, stress, overall microstructure and surface roughness. Defects are important and difficult to tailor for films. Typically, the features that make up defects are vacancies, where there is an absence of an atom at a lattice site, interstitials, substitutions, edge or screw dislocations, shown in Figure 3.2, and grain boundaries. Defects, and their density within a film, help determine the overall performance of a film in many respects. A large number of grain boundaries and vacancies, for example, could result in lower than desired electrical or superconducting properties. Defects are almost always undesirable features to have within a film, although it is not possible to eliminate them completely. Stress is important due to the amount of stored energy contained within and could be a result of lattice mismatch between the film and substrate or defects such as dislocations within the film. One possible negative effect of large amounts of stress is, in the presence of poor adhesion, the possibility of a film peeling from the substrate over time from the stress, exemplified by the low energy deposition $\mathrm{Nb}$ film peeling from $\mathrm{Cu}$ cavities discussed in Chapter 1.

\subsection{Film Structure}

Microstructure comprises several aspects of thin films like lattice parameter, grain size, grain structure and grain density. The lattice parameter can be key parameter in many studies, especially in relation to the superconducting properties, especially in binary or ternary element phases, since it is fundamentally a lattice phenomenon. In the latter 
case, lattice parameter is influenced heavily by actual stoichiometry, film stress and defect density [1]. Grains are prominent features within many polycrystalline thin films as films grow, especially during island growth, grains begin to form. When these features coalesce, a boundary can be created between the grains that are made of two rows of atoms which are weakly bonded to each other, making them more energetic. Since the atoms in grain boundaries are more energetic and thin films typically have a much larger number of exposed grains and grain boundaries than their bulk counterparts, thin films are more chemically reactive than bulk materials [2] [1].

Surface morphology has entirely to do with the properties of the evolving film surface during growth. In general, surface morphology is measured on a global scale, as RMS or average roughness and, as discussed earlier, the optimal roughness and features are highly dependent upon the application. One key aspect to keep in mind throughout this discussion of growth modes and resulting properties is the relationship of these aspects upon the conditions during deposition. A key parameter for deposition is the energy within the system, which can come in the form of substrate temperature or impinging atom energy, typically from ion bombardment and substrate bias. A useful tool to categorize this relationship is found in film structure-zone-diagrams (SZDs). Figure 3.3 shows an SZD where one can observe the evolution of film properties from porous, tapered crystals at low deposition energy toward a flip in stress and the creation of densely-packed, fibrous grains in the intermediate energy and finally to columnar grains and even recrystallization at high enough energies [29]. Diagrams like this reveal how 
important a role energetic considerations play during deposition and the multitude of opportunities to tailor the final properties within the deposition parameter space.

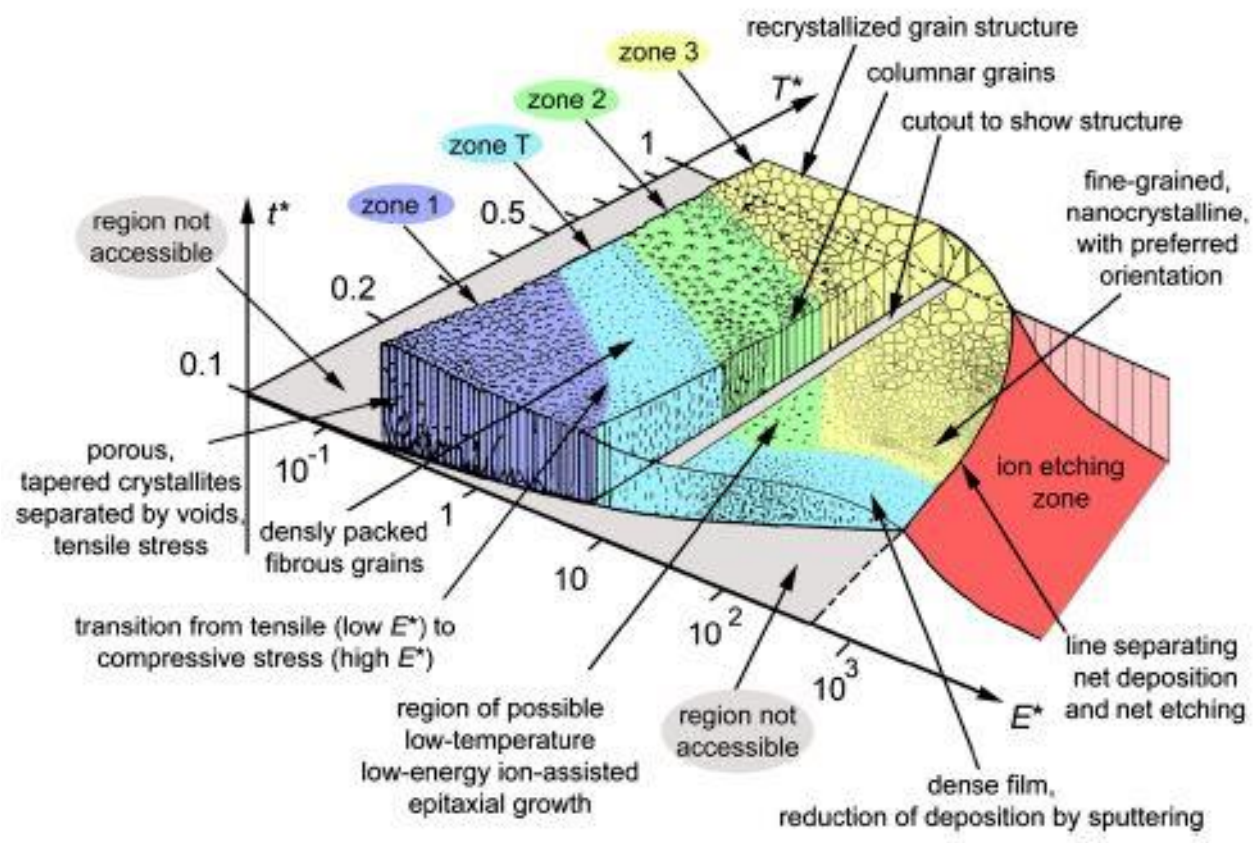

Figure 3.3: Modified SZD diagram. (reproduced from [29] with permission from the author and Elsevier Publishers)

To end this section, we will briefly discuss some of the advantages of using thin film technology in applications, the challenges associated with it and the specific nature of its implementation to SRF. Thin films in general offer many advantages over using bulk materials. They typically involve much lower manufacturing cost, as they use much less material than the bulk case. However, perhaps the biggest advantage over bulk materials is the access to deposition parameters. Having control over the deposition process offers significant advantages. First, it not only allows the tailoring of material properties for specific applications, it also allows for a high level of reproducibility in the resulting film 
performance, since parameters are calibrated periodically to ensure consistency of products. As an example, in typical magnetron sputtering performed in this research, some of the main parameters that can be controlled to affect film properties are pressure, sputtering power, substrate temperature and ion energy. While the sputtering power is usually responsible for deposition rate, the other three variables all have an important role to play in the energy of the deposition. For example, pressure affects the mean free path of the atoms in the system to the extent where high pressures can result in large numbers of atoms being scattered before reaching the surface, thereby reducing the energy of the atoms at the surface, while, conversely, a low pressure can lead to higherenergy bombardment of the surface and denser films.

While thin films offer many advantages, there are also challenges that must be overcome. Many of the challenges in thin film creation are related to the ability to achieve the desired film quality. Necessarily, thin films are different than their bulk counterparts - they are typically less dense, have more grain boundaries and can differ in microstructure from their bulk phase due to stress and defects. Also, thin films can have varying thicknesses across large surfaces, be difficult to deposit (depending upon the technique used) on non-planar geometries and can exhibit a miss-cut wherein the film layers do not grow parallel to the substrate surface, resulting in a misalignment between film and substrate. It is a constant process in thin film development to create substrate surfaces as ideal as possible for film growth and to alter deposition parameters to converge upon the best possible set of film properties for the desired application. 
Throughout this section we have discussed some of the fundamentals behind thin films and some of the appeal of using thin films. Specifically, for SRF applications, thin films are of interest due to their reproducibility during deposition, especially in the context of possible cavity manufacturing using this technique, the ability to engineer the active RF surface layer with new materials and the ability to control the thickness of films on the nanometer level. The latter is especially important for the application of the multilayer model based upon the increase in a superconductor's lower critical field with decreasing thickness below its London penetration depth. The films required for SRF application would necessarily have as bulk-like as possible microstructure, as superconductivity is a lattice phenomenon, a low defect density and a smooth surface with no sharp features that can lead to field emission or local field enhancement resulting in early flux penetration. 


\section{CHAPTER 4}

\section{Experimental Methods}

Within experimental physics there are certain techniques or equipment that are necessary to create samples and perform specific measurements. Experimentation requires controlled conditions in which to perform specific tasks or measurements reproducibly, while holding as many other variables as constant as possible. The thin film and condensed matter branch of physics utilizes a wide variety of specialized equipment and measurement techniques such as vacuum and ultra-high vacuum technology, X-ray diffraction, various electron microscopies and superconducting technology among many more. In this section, we will expand more upon the experimental tools and techniques applied to thin films used for the research discussed here.

\subsection{Vacuum Technology}

One of the most widely used techniques across many disciplines of science and industry is that of vacuum technology. Vacuum technology is an vital tool for research when highly-controlled and clean conditions are required for reproducibility, as well as for techniques that require large mean free paths of particles. Vacuum systems can vary dramatically based upon the intended application, from basic vacuum used for bell jar experiments on sound wave propagation, or lack thereof, to ultra-high vacuum (UHV) systems capable of reaching pressures of $10^{-9}-10^{-11}$ Torr (on the scale of trillion times 
lower than atmospheric pressure of 760 Torr). Vacuum systems today are capable of extremely low pressures and thereby offer conditions otherwise only found in the vacuum of space. These low pressures allow the capability to use many techniques not possible otherwise such as: particle accelerators, film deposition, electron microscopy and many spectroscopic techniques where extremely long mean free paths are required or extremely small signals must be discerned and low background noise is essential.

\subsubsection{Vacuum Hardware}

Fundamentally, vacuum technology involves designing systems capable of being airtight and installing pumping systems to evacuate the spaces of as much gas as possible. The basic vacuum equipment includes chambers made of metal or glass where connection between parts is done with flanges. For vacuum purposes, these flanges must be specially designed to keep a good seal from the outside atmospheric pressure always attempting to fill the vacuum created. There are two widely used flange types used in today's vacuum industry, KF and CF flanges. KF flanges are based off a simple O-ring style seal in which two flanges are connected with an O-ring gasket in between them; pressure upon each side of the flange forces them together, thereby compressing the rubber-like O-ring and creating an air tight seal. KF flanges are widely used due to their ease of operation and installation, as well as the ability to reuse the O-ring gaskets. However, KF flanges are only good in the high vacuum (HV) regime. Once a low enough pressure has been reached, the O-rings' own partial pressure in vacuum and the microscopic imperfections in the seal begin to limit the achievable pressure possible in the system. 
While KF flanges have their place in many vacuum systems where high vacuum levels are all that is needed or in the forepumping sections of UHV systems, for more demanding applications one must go to a different method of vacuum sealing.

The most widely used ultra-high vacuum flange sealing technique is that of conflat (CF) flanges. CF flanges work based on the ingenious and simple idea of using two flanges, made of much stronger material than the gasket, with so-called "knife-edges" (sharp ridges rising slightly above the flange faces) machined into their surfaces that allow for digging into the gasket material as the flange is tightened and creating a vacuum-tight seal. Typically, the flanges are made of strong stainless steel with copper used as the softer gasket material. It is important to note that the copper gaskets used for this technique require specific softness ratings such that the stainless steel flanges may dig into them deeply with the knife edges as the flange is tightened. If the copper is too hard, it may require too much force in order to tighten the flange properly or not be possible such that the connection does not hold good vacuum. CF flange technology has been proven to provide excellent vacuum sealing for applications demanding ultra-high vacuum.

Along with the excellent vacuum seal, CF flanges hold a plethora of benefits compared to KF flanges. CF flanges, since they are made of stainless steel and copper, can be baked up to respectable temperatures (limited by the copper to less than $450^{\circ} \mathrm{C}$ ), thus allowing for removal of adsorbed gases, such as water, from the surfaces of the vacuum system. Also, they do not outgas into the vacuum chamber, since the copper used for these applications undergoes special processing, like heat treatments, and, once 
connected, CF flanges last until the user decides to undo the connection. While CF flanges have many benefits, there are also drawbacks to consider. One major inconvenience of CF flanges is the fact that the gaskets are single-use consumables and thus, for applications requiring a large number of flange openings, can create a large amount of copper recycling along with being somewhat expensive. Also, for many applications including particle accelerators - copper gaskets are not suitable for other reasons. Within particle accelerators, a different technique of indium sealing is used for the vacuum flange connections since the copper gaskets cannot withstand the radiation for long periods of time and will eventually degrade into the vacuum system, potentially creating leaks. However, even with these disadvantages, CF flanges are still one of the most useful tools for creating UHV for scientific research systems.

Next, vacuum systems are made up of chambers and pipes welded together such that all the processes desired are able to be performed within them. Typically, a vacuum system is made up of a main chamber which is the centerpiece of the system (although some systems may contain multiple main chambers); this is normally where the processes for which the system was designed take place. Therefore, the choice of main chambers is an extremely important decision when designing any vacuum system to the extent that most vacuum systems are built around the chosen main chamber. There are many types of vacuum chambers in existence, such as bell jars and large stainless steel chambers, and many are designed with a specific intended application in mind, although they can be adapted for other uses as well. 


\subsubsection{Vacuum Pumps}

The next most important piece of equipment in any vacuum system is the pumping system. The choice of types of pumps is extremely important. Many pumps are specifically designed for certain applications and if used incorrectly can lead to damage of the vacuum equipment. Among the main types of pumps we can mention forepumps, turbomolecular pumps and trapping pumps. Forepumps, or roughing pumps, typically come in two different styles, dry and oil-based. Oil-based roughing pumps have been in use for as long as vacuum systems have been around and have been an invaluable tool for vacuum technology. They typically can achieve very good rough vacuum levels on the order of the mTorr range and can achieve their base pressures quickly even for large volumes. However, the major drawback to oil pumps is the great care and safety required for use in clean vacuum systems. If the oil pumps ever see a pressure gradient leading back into the vacuum system, such as if a power failure occurred shutting off all pumps at low pressure, the oil within the pump can be sucked back into the system, thus ruining the vacuum quality inside.

Oil is a major enemy of vacuum systems achieving very low pressures since it has a large partial pressure in vacuum and can prevent systems from reaching their desired pressures. Oil is such an issue that users typically wear long sleeves and latex gloves when working on vacuum surfaces to not allow even oil from a human finger to be exposed within the vacuum system. In order to reduce the possible damage from catastrophic events where oil migration may occur, many systems are employed such as oil traps, 
molecular sieves and solenoid valves that seal automatically when power failures occur. While these techniques are good, there is no fullproof way to prevent contamination when using oil pumps and more importantly, these techniques can be expensive and depend largely upon the user to follow best practices when working with the vacuum equipment. Therefore, new techniques were developed that remove the oil from the forepump systems altogether. Roughing pumps that do not utilize oil are called dry vacuum pumps, one example of which is a scroll pump. Scroll pumps are extremely useful pieces of vacuum pump technology and allow the development of vacuum systems that are more user-friendly and do not require expensive safety apparatuses to prevent contamination of the vacuum system. However, dry vacuum pumps are typically much more expensive than their oil counterparts and can be cost-prohibitive, depending upon the project.

When going to higher vacuum than achievable by forepumps alone, the next level of vacuum pumping hardware can be that of turbomolecular pumps. Turbomolecular pumps are essentially jet engines without fuel. That is, they work on the principle of using highly-engineered turbines that are extremely close together and, when rotated together on low friction bearings, or even magnetic levitation, can create a large pressure gradient from the inlet to outlet, thus pumping gas from the vacuum chamber. Turbo pumps are widely used in the vacuum industry and come in many sizes and designs. Turbo pumps typically cannot operate at pressures above the $10^{-2}$ Torr range and thus require forepumps to create the initial vacuum and maintain a good outlet pressure. When the pressure gets too high, the drag force created by the rotors spinning in the gases can 
cause the turbo pumps to get hot, so many require air or even water cooling to operate, even for operation in high vacuum. Due to the heat, the blades can expand and, since the blades have extremely small clearances between them, can cause catastrophic failure by making contact and possibly destroying the entire inside of the turbo pump. Therefore, it is important to not only operate the pump in the specified pressure range, but also to ensure the proper cooling system is being used and is operating appropriately. Turbo pumps have many uses and are specifically good for use in PVD thin film techniques since they can operate well in the mTorr range which many of these processes require and are not at risk for damage in this regime.

One wildly different technique for vacuum pumping that has been used for a long time is that of gas trapping. Gas trapping is straightforward in concept - it mainly involves the trapping of gas in some medium that either keeps the gas trapped there when in use or can be sealed off and the gas removed from the system altogether. Gas trapping pumps are by far the best and preferred method for achieving ultra-high vacuum pressures. The three main types of gas trapping pumps are sorption pumps, cryo-pumps and ion pumps. Sorption pumps have been in use for a long time in vacuum technology and are useful since they can not only achieve very good pressures but also have high pumping speeds and can be used for the initial evacuation of the system. Sorption pumps work by having a canister, or multiple canisters for higher pumping speed, full of a gas-trapping medium attached to the vacuum system with valves capable of sealing the canisters off from the system. When the medium is cooled down to cryogenic temperatures, typically that of liquid nitrogen, the medium traps gas that it is then exposed. This allows for an iterative 
system in which the pumps are opened to the chamber and cooled down, thus trapping gas, and then sealed off from the system and warmed up in air to release the trapped gas from the system. This cycle is repeated as many times as desired, allowing for very low pressures to be achieved. However, this technique can be very time consuming, with some modern systems utilizing computer control to perform the process.

Once cryogenic technology advanced and allowed for the compression and cooling of helium, many new techniques have become available. One gas trapping pump that utilizes such helium compression and cooling is a cryo-pump. Cryo-pumps work by a similar technique as sorption pumps, that is, a medium that is cooled and traps gas; however cryo-pumps do not remove the gas from the vacuum system, but instead trap the gas within the surfaces of the pump, which prevents the gas from being free in the system. Cryo-pumps are extremely useful vacuum pumps and are in wide use for applications that require extremely fast pumping speeds or UHV pressures. However, cryo-pumps suffer from the fact that, because they do not remove gas but rather trap it in their surface, they are limited in the maximum amount of gas that can be trapped and therefore the amount of pumping possible. As cryo-pumps are used, they continue to trap more and more gas, and once the maximum amount is reached, the mass of the pump that needs to be cooled exceeds the cooling capabilities of the compression system, thus raising the temperature and releasing gas back into the system, or at least not being able to pump further. Once this point is reached, one must perform a regeneration cycle in which the pump is warmed up to room temperature and the trapped gas allowed to go back into the system, ideally to be removed by other pumps in the system. Cryo-pumps 
are constantly a battle of trade-offs based upon the desired operation lifetime between regenerations. Typically, cryo-pumps can be turned on in the mTorr range of pressure, but the higher pressure to which the cryo-pumps are exposed, the more gas they trap and the sooner they require regeneration. Also, cryo-pumps can be highly valuable for sputtering techniques due to their high pumping speeds and ability to be throttled by valves, but again the more pressures they are exposed to, the more often they must be regenerated, which can be a multi-day process. Even with these drawbacks, cryo-pumps are an invaluable tool for scientific research systems requiring UHV pressures and were employed in the cavity deposition system used in this research.

Finally, the third type of gas trapping pump to be discussed here is that of ion pumps. Ion pumps work similarly to cryo-pumps in that they do not remove gas from the system, but instead trap the gas within the pump itself, removing it from within the vacuum system. Ion pumps work by ionizing gas within the vacuum system and then using a strong electric field to accelerate the ion down to a cathode where the gas is trapped during operation. Ion pumps are widely used in many applications and are typically capable of extremely good pressures in the $10^{-11}$ Torr range. One of the main benefits and uses for ion pumps is in that of vibration sensitive applications like some microscopy techniques, such as precise electron microscopies, in which vibration presents as background noise and can be harmful to the quality of data being collected. There are no moving parts in ion pumps - they are exclusively electrostatic pieces of equipment and therefore do not create noticeable vibrations as compared to turbo pumps and cryopumps. Also, since gas is trapped within the system, once the system has reached a 
suitable pressure for operation of the ion pump, the system may be sealed and all other pumps turned off to eliminate them as sources of vibration. Along with very highprecision measurements, ion pumps are also in wide use in particle accelerators as vibrations from pumps can affect the performance of the SRF components and therefore the quality of the beam.

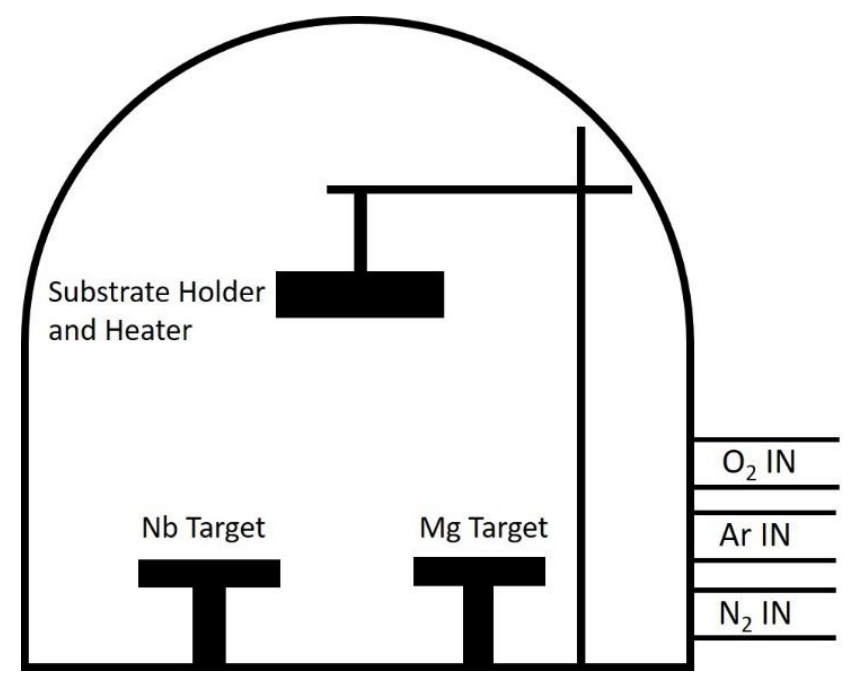

Figure 4.1: Simplified diagram of bell jar deposition system used for NbN and NbTiN depositions.

With respect to the research presented in this thesis, two systems were used: one for the deposition of $\mathrm{NbN}, \mathrm{NbTiN}$ and multilayer films and the other for the deposition of $\mathrm{Nb}$ films onto small samples and SRF cavities. The multilayer deposition system is a bell jar type system, shown pictorially in Figure 4.1, with a Viton O-ring seal for the main chamber. The bell-jar system utilizes an oil-based roughing pump and a large turbomolecular pump for high vacuum pressure capable of reaching the $10^{-7}-10^{-8}$ Torr range. The cavity deposition system, schematically shown in Figure 4.2, was designed specifically for research into deposition of $\mathrm{Nb} / \mathrm{Cu}$ cavity coatings with the ability to deposit 
cavities and small samples without ever having to vent the overall system to atmosphere.

This system was designed with UHV in mind and therefore all vacuum connections upstream of the roughing pump lines are CF flanges and the system was rigorously cleaned and then assembled in the JLab production clean room (rated at ISO-4). For pumping the system, a dry scroll pump was used for the rough pumping followed by a small turbomolecular pump for pumping to the HV-UHV range and then a cryo-pump was used to achieve the final UHV background pressures in the $10^{-9}-10^{-10}$ Torr range.

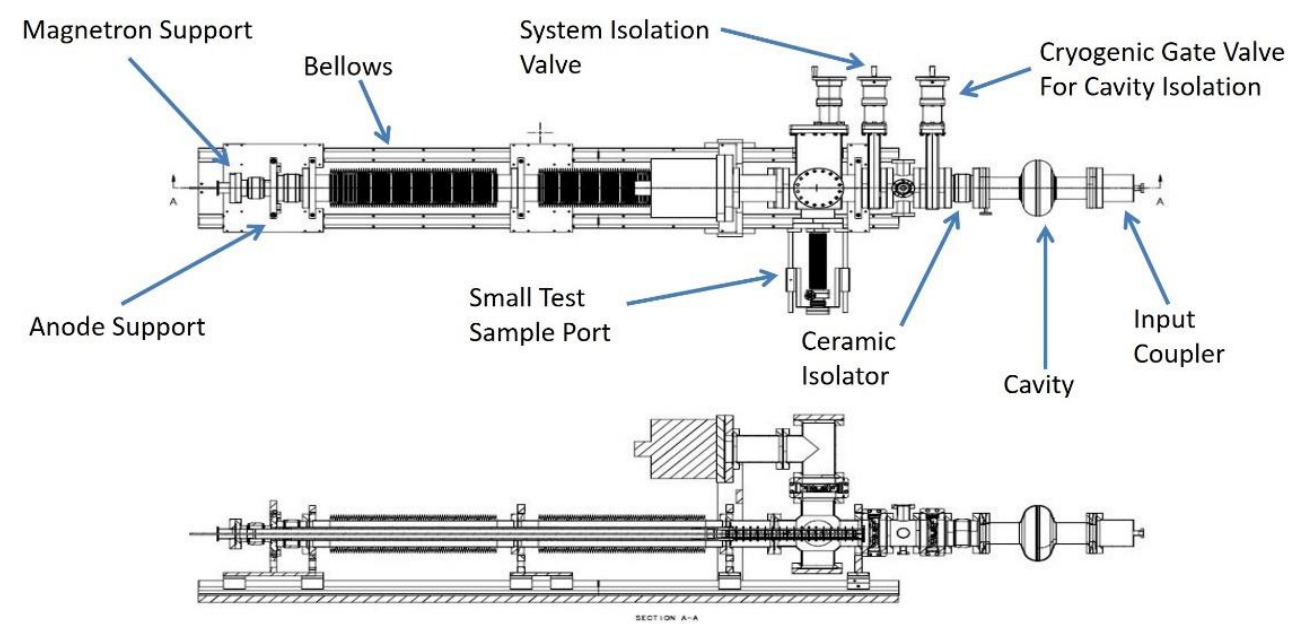

Figure 4.2: Schematic drawing of JLab cavity deposition system.

\subsubsection{Vacuum Measurement}

To characterize the effectiveness of vacuum seals, the pressure and cleanliness of a vacuum system, etc., vacuum measurement and diagnostics techniques are needed. Vacuum measurement can be broken up into two main categories, general pressure measurement and gas species analysis. Both techniques are essential for effective 
commissioning and use of any vacuum system. There are a wide variety of techniques for pressure measurement and vacuum diagnostics that are beyond the scope of this thesis, however, there are a great number of resources available that help in choosing the correct pressure gauge and necessary diagnostics based upon the requirements of the system and the application [1] [2] [3] [4]. Here we will discuss vacuum diagnostics and pressure measurement both in general and in regards to the effective use of thin film deposition systems.

Pressure measurement is perhaps the most important diagnostic or sensor within any vacuum system. Based upon the pressure of the vacuum system one can instantaneously detect whether or not a problem exists and what, if any, further methods are needed to diagnose and fix the issues. Pressure measurement is a very broad area in which there are numerous different methods used to accomplish the task. Some examples of vacuum measurement gauges using different techniques are vacuum ionization gauges, Pirani gauges, cold cathodes, capacitance manometers and Convectron $^{\circledR}$ gauges. While most gauges are sound in their measurement capabilities, they can vary dramatically in their ability to accurately measure pressure within specific ranges. For example, capacitance manometers can be designed to be highly precise at measuring pressure from atmospheric levels down to $10^{-2}-10^{-3}$ Torr, but below this level they become unable to measure the pressure accurately and therefore should not be used within this regime. On the other hand, vacuum ionization gauges, or "ion gauges", such as Bayard-Alpert gauges, are extremely precise and effective at measuring pressure in the HV to UHV range and are typically the gauge of choice for this regime. However, 
ion gauges are very sensitive to high pressures and can become damaged if operated at too high of a pressure. For most ion gauges, the controllers have safety settings that turn the gauge off once a threshold voltage is reached. This is of extreme importance when picking a gauge for thin film deposition systems, since most vacuum deposition techniques require gas pressures to be in the mTorr range; as a result, the ion gauges cannot be used for monitoring process pressure for these deposition techniques. Usually, a system will require multiple gauges in order to read the pressure across the full range of operation and one should think carefully and plan accordingly for what gauges are needed in the system and where they should be installed physically on the system to account for possible conductance or film coating areas.

Along with pressure measurement, another important diagnostic tool within any vacuum system is that of gas species identification or sensing. One of the main applications of this is for a technique called helium leak checking, a method by which one can spray vacuum hardware connections with helium gas and, since helium gas can migrate very effectively through small cracks, sense the presence of helium gas that has leaked into the system. This technique is invaluable for determining if the flange connections and vacuum hardware are assembled properly and able to hold good vacuum. While there are diagnostic systems dedicated to helium leak checking that can be very sensitive, a useful tool that can be used for He and other gas species is a residual gas analyzer (RGA). An RGA uses a mass spectrometer to ionize and analyze gas within a vacuum system; the specific ranges of gas that can be detected are dependent on the RGA model. This can be useful for a variety of purposes within a vacuum system such as 
the aforementioned helium leak checking, as well as contaminant analysis, vacuum cleanliness and even determining the purity of gas input lines.

RGAs typically require good pressure in order to operate, so as to extend the lifetime and not damage the filament present within them, although some models can be purchased that are differentially pumped so that higher pressure processes may also be analyzed in real time using the system. As mentioned above, along with helium leak checking, two important tasks RGAs are commonly used for are process gas analysis and system cleanliness. When performing PVD within a vacuum system one typically employs the use of a working gas, typically a noble gas. However, one requires high-purity gas for these processes and contaminants such as air can have deleterious effects on the process being used. One method for determining the purity of gas lines is to input a very small pressure from the lines and analyze the change in gases present in the system before and during gas input. This gives insight into the purity of the gas being used and any potential problems with the gas lines not being sealed properly. Another very important technique is the analysis of the background gases in a vacuum system. Using an RGA it is possible to detect the presence of hydrocarbons, such as oil residue in the system, and water vapor which both have detrimental effects on the ultimate vacuum quality one can achieve.

Within the vacuum systems used for this research, several different vacuum gauges and diagnostics were employed. The bell-jar system used for multilayer studies utilized a Pirani pressure gauge good over a wide range of pressures. The JLab cavity deposition system had a cold cathode gauge for sub-atmospheric to HV pressure and for sputtering gas pressure monitoring and a Bayard-Alpert nude ion gauge for HV to UHV 
pressure measurement. Also, the cavity system incorporated the use of an RGA installed on the system for analysis of gas species and for leak checking the system.

\subsection{Thin Film Deposition Techniques}

Thin films are a powerful tool in many modern technological and industrial practices, along with being instrumental in scientific endeavors. Here we will focus on the practical techniques employed in general for thin film deposition, as well as focusing in more detail on the specific techniques employed for the work done in this thesis. Thin film deposition is a widely-used tool in a broad range of industries and the ability to understand and implement deposition techniques is in and of itself an extremely important skillset.

As mentioned previously, there are many different techniques that can be employed for the creation of thin films on surfaces; these can essentially be classified as either CVD and PVD. There is a large array of widely varied techniques contained in each of these categories, as well as hybrid techniques that can utilize methods from each. CVD techniques, while not used in this research, yield a significant percentage of thin films created across the world and are typically categorized by the conditions used for deposition such as the pressure range or heating method, among others. Fundamentally, CVD techniques employ the use of gases, or precursors, that when used in a certain order or in certain situations, chemically react or decompose and result in the material desired condensing on surfaces. 


\subsubsection{Chemical Vapor Deposition}

CVD is very useful for an array of reasons. First, due to the gaseous nature of the process, complex geometries can be coated easily and quickly, assuming one has the appropriate equipment. Second, CVD techniques typically have very high deposition rates and offer conformal coverage of the desired surfaces which is very useful for industry or in applications where process time and reliability is very important. Finally, CVD techniques have the ability to deposit a wide range of materials and have very precise stoichiometric control over the film throughout the process. Atomic layer deposition (ALD), a specific CVD technique, holds great promise for many applications where highly precise stoichiometry and thickness control is needed, such as for the SIS multilayer structures mentioned earlier. ALD utilizes a cyclical method, shown in Figure 4.3, of inputting precursor and reactant gases that undergo a self-limiting reaction on the substrate surface, only allowing one monolayer of the film to be created. As a result, ALD provides precise control over the thickness of the film by having one cycle per monolayer. One can also alternate the monolayers in precise ratios in order to obtain nearly perfect film stoichiometry. 


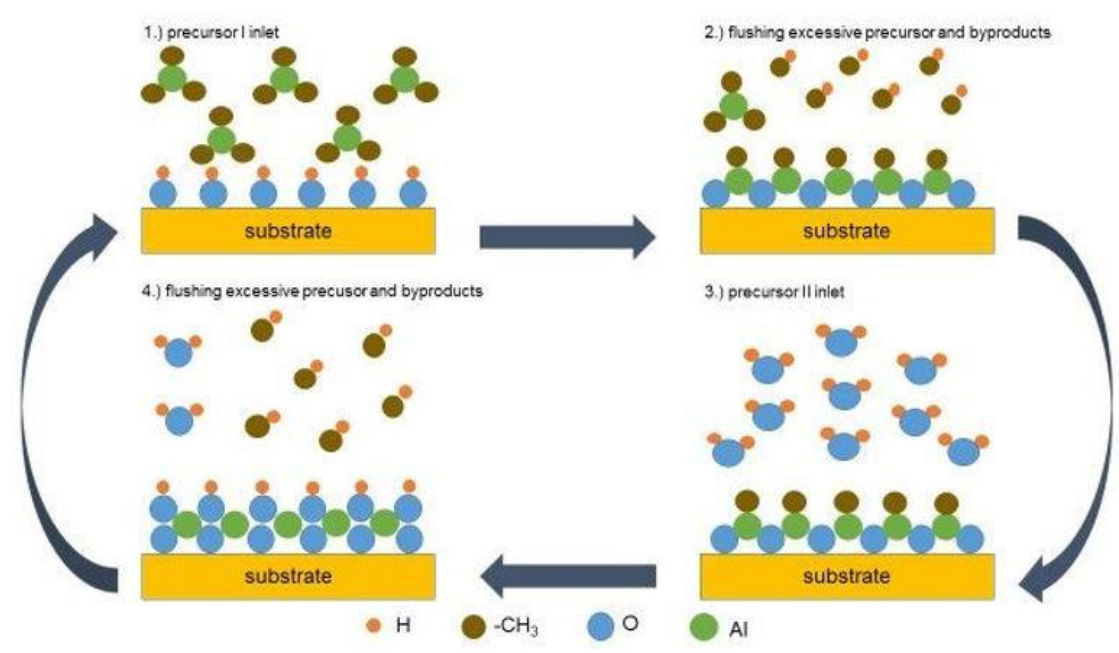

Figure 4.3: Simplified ALD steps showing cyclical nature of process. Image from: https://www.uni-due.de.

While CVD has many beneficial elements, as with any process, there are drawbacks. One of the main drawbacks to CVD is the reliance of deposition on the availability or existence of precursors. While CVD has access to an extensive library of materials, there are still a large number of materials that are not currently available. Along with the lack of certain necessary types, many CVD precursors and exhaust gases, can be highly toxic and damaging to the environment or human health. This requires extensive safety protocols when handling such mixtures and disposal of the waste created from the CVD process. While many reasonable safety protocols can be implemented without much difficulty, this can pose a serious hurdle to overcome, especially for smaller scale operations. Even with the potentially hazardous nature, the benefits of CVD, along with the relatively affordable equipment and operation, have made CVD a highly sought-after and desired deposition technique throughout industry and academia. 


\subsubsection{Physical Vapor Deposition}

The other category of thin film deposition techniques is that of PVD. There are a variety of techniques that fall under the PVD heading. Broadly, PVD techniques involve the removal of a material from a solid form followed by vaporization of the removed material. This vapor expands into the vacuum and then condenses on surfaces within the chamber and builds up over time into a solid film. While each technique under PVD shares this principle, it is somewhat deceiving to think that all of the techniques are similar. There is a large variety in methods with which to remove the material from a solid and turn it into a vapor. The method by which the material is formed is the main differentiation between the techniques, although there are other properties of the various techniques that play an important role in film growth. PVD contains a large number of techniques, some of which we will discuss briefly, such as laser ablation, electron beam evaporation, molecular beam epitaxy and sputtering. However, the remaining majority of this chapter will be dedicated to the sputtering techniques, specifically DC methods, due to their relation to the work done within this thesis.

Laser ablation is a technique by which a target is impinged upon by a strong laser, typically originating from outside the vacuum chamber. When the laser hits the target, the laser ablates the material - energy transferred from the laser forces material out of the surface and into the vacuum as a vapor cloud. Laser ablation benefits from the fact that the laser removes material independent of the material type, leading to very good stoichiometry mapping from the target to the substrate, which can be very useful when 
desiring to sputter alloys from a bulk target. Also, laser ablation can be done in high vacuum since there is no working gas involved in the process. This allows for highly pure films to be created with very few contaminants and little gas burial. However, laser ablation suffers from some key drawbacks. First, it requires specific geometry in order for the laser to be able to strike the necessary target and coat the desired surface. Second, since the laser is a very brute-force method, there is a risk of removing macroparticles from the target that are not individual atoms. These, when landing on the substrate, can be a defect starting point and detrimentally effect the entire film.

Another high vacuum PVD technique is that of electron beam evaporation. Electron beam evaporation is a technique that has been in wide use for many decades. On a basic level, electron beam evaporation involves the placing of a target material in pellet form in a crucible and then using magnetic fields to direct an electron beam into the crucible. As the electron beam strikes the target material it heats the material, to the point at which it begins to evaporate and spread into the vacuum as a vapor. This vapor can then condense on surfaces, creating thin films. While electron beam evaporation shares many similarities to laser ablation, it benefits mainly from the ability to remove individual atoms from the target while not having the risk of removing macroparticles. However, electron beam evaporation requires generous geometry characteristics of the vacuum chamber and fairly large thermal cooling investment due to the elevated temperatures created, which if not properly operated can cause severe damage to not only the films being created but also the vacuum system hardware. While electron beam evaporation is not as widely used in industrial applications, it is still in use in many 
academic settings and it is a useful tool in creating a source for other deposition techniques such as electron cyclotron resonance (ECR) to be discussed later in this chapter. Molecular beam epitaxy is similar to electron beam evaporation, except the crucibles holding the material are heated directly. While we did not go into any of these techniques in great detail the interested reader can access many sources for more information on these and other techniques [1] [2] [3] [4].

\subsubsection{Sputtering}

The last technique to be discussed in this chapter is that of sputtering. While sputtering is itself a broad term with a rich variety of techniques contained therein, the underlying principle of material removal is shared between them all. This section is of utmost importance for this thesis since all the films studied herein were deposited using a form of sputtering. Sputtering can take many forms which can be broken up into four main types: direct current (DC), DC magnetron (DCMS), pulsed DC (MS) and alternating current (AC/RF).

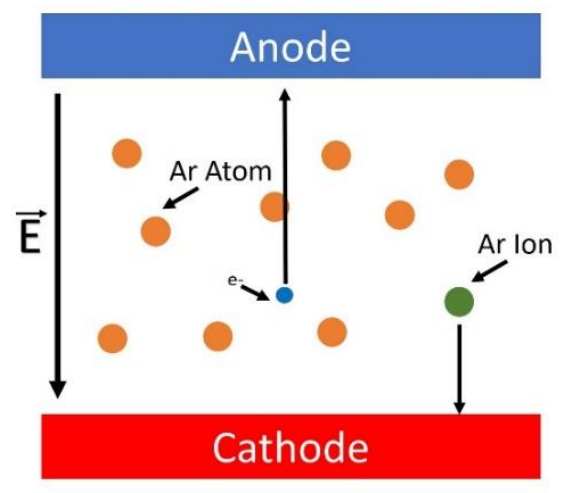

Figure 4.4: Diagram of DC sputtering showcasing the ionization of sputter gas atoms (Ar) by free electrons and the acceleration of Ar ions toward cathode target ejecting target material and secondary electrons. 
Fundamentally, sputtering is the removal of matrix atoms from a bulk target material by collision with another atom or ion. The main way, shown in Figure 4.4 , that this is accomplished in practice is by the ionization of gas atoms, typically noble gases, via electron collisions within a vacuum system by applying a voltage between an anode and cathode. The ions are then accelerated toward the cathode target, where secondary electrons are produced, leading to a cascade of gas ionization and eventually ignition of a plasma between the anode and cathode. Once a plasma has been formed, a current develops between the anode and cathode as a result of the now large number of ions impinging upon the target surface. As the ions bombard the target, some number of the target atoms can be removed by the collisions and eject the atoms into the vacuum. These "sputtered" atoms are now able to expand into the vacuum chamber and coat the surfaces contained therein. The number of atoms ejected per incident ion striking the target surface is defined as the sputter yield:

$$
\text { Sputtering Yield }=\frac{\# \text { Sputtered Atoms }}{\text { Incident Particle }} \text {. }
$$

Sputter yield is an important value and is strongly dependent upon the gas type being used, such as argon ( $\mathrm{Ar}$ ) or Krypton $(\mathrm{Kr})$, and the target material. Certain gases are more effective at sputtering different target materials based upon their relationship between mass and size. Therefore, it is important to determine the type of gas that is most appropriate for the sputtering target material. Typically, one would prefer to have the gas atoms as close in mass to the target atoms as possible for the most efficient 
energy transfer and therefore best sputtering efficiency. Sputtering has shown to be very useful due to its high deposition rates and stoichiometry mapping from target to film.

Another tool available in thin film deposition, the importance of which will become clearer later when the ionized deposition techniques are discussed, is that of ion bombardment. As we can see above, the process of ion bombardment is responsible for the sputtering of the target material, but ion bombardment is also very important for the quality of the films being deposited throughout the growth. Ion bombardment of growing films can alter many properties of growing films, including surface topography and roughness, crystallography and texture, grain structure and the defects and stress in the films. Ions that are incident upon any surface (here, of course, we are concerned with only the film surface) can adsorb (stick), scatter, sputter or implant. Which of these outcomes occurs is typically determined statistically, but also relies heavily on the kinetics of the impinging ions, such as their energy and incident angle. For example, low energy ions typically lead to condensation of the atoms on the surface, while very high-energy ions contain enough energy to implant themselves deep within the surface, which results in excellent adhesion while not causing re-sputtering of the material during the process, but perhaps also inducing subsurface structural defects.

Now that we have discussed how sputtering in general works and the benefits of ions for film deposition and growth, we can spend a short time discussing some of the specific methods by which sputtering is induced. DC sputtering is very similar to the process outlined within the general sputtering overview above. Traditional DC sputtering requires large voltages and high pressures in order to create the plasma required for 
sputtering. While DC sputtering was used for many years and resulted in great scientific advancement, the high voltages and high pressures had many drawbacks. First, high pressures not only lead to lower deposition rates due to the low mean free path of the sputtered atoms, but they also result in a large number of gas atoms becoming trapped or buried within the growing film that act as defects in the film. This can cause stress and affect the properties of the resulting film.

While DC sputtering is very effective for use in creating metal films, it has difficulties when attempted on materials with lower conductivities. As the conductivity of the target is lowered, especially in reactive sputtering, the target can have charge accumulation on the surface that builds up over time. As the charge builds, up it can lead to unipolar or bipolar arcing within the system that erodes the target surface via cratering and can eject macroscopic droplets into the vacuum that settle onto the substrate or film surface and are incorporated as defects within the film. Since this process can be so detrimental to film growth and quality, a process modification is needed. To overcome this problem, the process of pulsing the DC power to the target was instituted. In principle, as the target power is pulsed, the charge accumulates during the on-time and discharges during the off-time, never allowing enough charge to accumulate on the surface to cause arcing. In practice, pulsed DC sputtering can be done as described or with the addition of a positive bias on the target so that during the off-time the positive charge accumulated is repelled by a positive potential and forced to leave the surface. Pulsed DC sputtering has proven very useful for sputtering of insulating materials, but perhaps more effective in the regime of reactive sputtering used in this research. 
The most effective method of sputtering insulating or non-conductive materials is that of $A C$, or RF, sputtering. AC sputtering is typically referred to as RF sputtering since the most common, FCC protected, frequency for plasma processing is around $13 \mathrm{MHz}$, within the RF regime. RF sputtering works based upon coupling of an RF power source to a gas within the vacuum. Instead of the linear cascade developed in DC sputtering that is heavily reliant upon secondary electrons created at the cathode, the electrons are oscillated back and forth within a small region of the vacuum and can be used multiple times for ionization since they do not migrate to the anode. As a result, a plasma is created that does not rely heavily upon the secondary electrons which are in shorter supply in low-conductivity materials. However, one question that can be asked is how do the ions escape the RF field and bombard the target surface to cause sputtering? This process is in fact based upon the mass difference, and resulting mobility difference, between the ions and the electrons.

Since the electrons are much lighter than the ions, they react more quickly to the alternating applied electric field while the ions move much more slowly in response due to their higher mass. Since there is now a difference in mobility, one side sees more charge throughout time than the other. However, since this is still fundamentally a capacitor system, no net charge flow can occur. This then requires that the target selfbias itself to a negative potential in order to compensate. This now negatively self-biased target attracts the ions from the plasma, resulting in sputtering. RF sputtering is a very useful technique, specifically for non-metallic targets, although it has low sputtering rates 
and is almost exclusively used in applications where the target material is low enough in conductivity that DC sputtering techniques are not very efficient.

A major improvement on traditional DC sputtering is the inclusion of a magnetic field along with the electric field which is referred to as DC magnetron sputtering (DCMS). The presence of the magnetic field causes the charged particles, especially electrons, to have a much larger path length en route to the anode due to the Lorentz force. This increased path length then increases their likelihood, or cross-section, for collision and ionization of gas atoms. While the magnetic field orientation can be either parallel or perpendicular to the applied electric field, it has been shown that the magnetic field perpendicular to the electric field is the ideal orientation since it not only causes long path lengths, but also confines electrons closer to the target surface and forces electrons to jump from side to side on the target, yielding a much greater cross-section. This has even been taken one step further in most modern sputtering setups to include toroidal magnetic fields from what is called a sputtering gun; this modification is very effective at trapping electrons and the plasma near the target surface and sputtering preferentially from a "racetrack" that is formed on the target surface.

DCMS has many benefits over traditional DC sputtering which is largely why DC sputtering has been completely replaced by DCMS in modern applications. First, DCMS allows the ignition of a plasma at much lower pressures, which results in a higher mean free path for the sputtered atoms, and therefore higher deposition rates, as well as fewer contaminants within the film as a result of gas burial. Second, the current is much higher at the same voltage for DCMS as compared to DC sputtering. This produces a plasma that 
is denser than that present in DC sputtering and with a higher ion content, with all the benefits therein. However, DCMS still has some concerns. First, since the magnetron removes material preferentially from the surface at the racetrack, the target is used up much more quickly than if the material was removed uniformly from the surface. This is an important concern for industry, where efficiency and cost are paramount. Second, magnetrons are prone to arcing, especially in reactive sputtering which can lead to contamination of or damage to the resulting films. Third, since the plasma bombardment is so great in DCMS, heat management is important; targets can arc or even crack if not cooled properly. Finally, uniformity is perhaps the most important concern with regard to DCMS. Since sputtering takes place in racetracks, there is an angle with which the target atoms are now ejected from the surface. This angular dependence plays an important role in applications where the substrates are non-planar such as micro-electronics with trenches and step-edges.

Along with straightforward DCMS of a target, whether it be an alloy or elemental, there is a special case of magnetron sputtering called reactive magnetron sputtering. Reactive magnetron sputtering allows the user to create film compositions and compounds in thin film form that may be difficult or impossible to create in bulk. Fundamentally, reactive magnetron sputtering is the sputtering of a metallic target in the presence of a reactive gas, such as oxygen or nitrogen. Using reactive sputtering, one can create films that are solid solution alloys of the target metal doped with the reactive element, a compound or a mixture of doped and compound materials. This spectrum of possible results is ideal for many modern applications as it allows the user to have fine 
control over composition and properties of the resulting film - truly surface engineering. One key feature of importance for reactive sputtering is the hysteretic behavior it exhibits in pressure, cathode voltage and deposition rate. Unlike the working gas, when the reactive gas is present, it is being consumed within the system via reactions at the target and the substrate. Therefore, the behavior at different points in the process is very different and can be observed through the hysteresis curves present. An important result of the hysteretic behavior is the necessity for fine process control throughout deposition, since the film properties are very sensitive to deposition conditions, and the constant monitoring of the system since many reactive gases can cause target poisoning by reducing the conductivity at the surface and leading to arcing and defects. The interested reader can pursue this topic further through many resources available since reactive sputtering is a rich field with many aspects to successful implementation [1] [2] [3] [4].

Finally, we will discuss the exciting and quickly developing field of energetic condensation and ionized physical vapor deposition (IPVD) techniques. As was mentioned previously, ion bombardment is an extraordinarily powerful tool for film deposition. Not only is it critical to sputtering, but it also has many beneficial effects on growing films. Many techniques over the years have taken advantage of the benefits of ion bombardment on growing films by using ion beams directed at the film surface and techniques such as unbalanced magnetron sputtering to direct more of the sputtering plasma content to the film surface. While these techniques have yielded films with significantly improved qualities, there is still a major failing inherent in the process - the ions used for film modification are typically dominated by the gas ions. While they may 
still yield films with improved properties, they also have the drawback of leading to high gas burial content within the film. This is where many new techniques have come in that allow for the creation of films using plasmas which are rich in sputtered metal ions. Having the ions being made up of the sputtered metal allows the same benefits of ion bombardment such as improved adhesion, microstructure, density and surface morphology, while limiting the amount of gas buried within the system. One key improvement of metal ion-rich plasmas is the ability to now direct the target material to coat non-uniform surfaces. This has been especially useful in the deposition of thin metallic films for use within the micro-electronics industry where it is desired to have the metal conformally fill trenches with large aspect ratios while not burying large amounts of working gas. Two such techniques that are gaining traction within the thin film field are that of electron cyclotron resonance (ECR) and high power impulse magnetron sputtering (HiPIMS). [30]

ECR is a fascinating technique that combines electron beam evaporation, to create a high vacuum cloud of metal atoms, and RF cyclotron resonance, to ionize the metal atoms. After ionization, there is essentially a monoenergetic beam of singly-ionized metal atoms that can be directed to substrate surfaces without the need for working gas. This technique holds great promise and has already been shown to yield very high-quality films [31]. However, ECR suffers from the complex geometries required to create the metal ion beam and the fact that, while it is effective at coating surfaces within a certain window, it is difficult to coat large three-dimensional surfaces such as SRF accelerator cavities. 
Once these technical challenges can be overcome, ECR will be a very useful technique for the development of high quality thin films, especially for SRF applications.

The second ionized deposition technique we will discuss is that of HiPIMS. HiPIMS is a specific form of pulsed DCMS which operates in the regime of extremely high peak power densities at low frequency and with short pulse width. HiPIMS works on the principle of applying a very large power density to the magnetron such that a plasma is created that is several orders of magnitude denser than that found in typical DCMS. The power densities are so high, however, that the power may only be maintained for very short periods of time and at low frequencies in order to keep the duty cycle low and manage thermal issues. As a result of the extremely dense plasma, the probability of ionization of sputtered species is extremely high and yields plasmas with a large percentage of metal ions - many materials are capable of achieving $50 \%$ metal ion fraction, with some reported up to $99 \%$ ionization fraction [32]. The high ion fraction allows for energy control of the deposited metal species leading to the ability to implement many of the benefits of ion bombardment and coating of non-planar surfaces [1] [30] [33] [34] [35] [36] [37].

While HiPIMS is a very promising technique, it still has some negative attributes that must be discussed. First, HiPIMS systems are pulsed at extremely high-power levels, which can be a safety hazard if not managed with appropriate safety controls, and the high-power pulses can be sensitive to system design, leading to arcing. Second, the low duty cycle that HiPIMS is limited to lower the average deposition rate to well below DCMS 
levels, although it also exhibits extremely high instantaneous deposition rates which can also lead to a large amount of trapped gas buried within the film.

Along with the high ion fraction achievable, HiPIMS has one major practical advantage over other IPVD techniques in that it can be readily adapted to use existing DCMS based sputtering systems. Typically, the only modification to a DCMS system needed is the addition of a new high-power pulsing supply. While the power supplies capable of HiPIMS are typically very expensive, it is also possible without deep knowledge of electrical engineering to create custom supplies for HiPIMS sputtering as was done for this research at JLab.

The JLab cavity deposition system was specifically designed for SRF cavity depositions with a movable cylindrical cathode and offers the ability to condition the target before depositions; load and coat small samples via a load-lock port; isolate each section of the system with gate valves, which is especially useful for the ability to load cavities without having to vent the whole system, thus allowing for easy maintenance of the UHV condition; and rotate between horizontal, for maintenance and mounting of cavities, and vertical, for cavity deposition, geometries, thus minimizing the risk of contaminants being able to fall on surface. The schematic shown in Figure 4.2, represents the early design of the system; since then, many improvements have been made to increase the conductance, chamber size, cleanliness, and clearances, thus reducing arcing risk. The HiPIMS system employed uses an $18 \mathrm{~kW}$ supply, with a 60A/208V wall supply, capable of pulsing the magnetron up to a peak power of $\sim 500 \mathrm{~kW}$ and was specially designed and built at JLab for this application. 
Throughout this thesis, multiple film deposition techniques were utilized for the creation of samples. The main type of deposition used for all the samples contained within was that of magnetron sputtering. Specifically, DC magnetron reactive sputtering in a belljar vacuum deposition system, shown pictorially in Figure 4.1, was used, for the creation of the $\mathrm{NbN}, \mathrm{NbTiN}$ and their multilayer films discussed in Chapter 5; this system has a base pressure of $10^{-7}-10^{-8}$ Torr and a heater capable of heating samples up to $\simeq 600^{\circ} \mathrm{C}$. The bell jar system was extremely useful for the multilayer work because of its use of multiple sputtering guns, allowing for different material layers to be deposited without venting to atmosphere, and for its quick turn-around of samples allowing for large calibration series to be performed prior to multilayer growth. For the $\mathrm{Nb} / \mathrm{Cu}$ work in Chapter 6, HiPIMS was used in the JLab cavity deposition system in order to explore the improvements of $\mathrm{Nb}$ films within the energetic condensation regime along with the possibility of obtaining RF results from coated SRF cavities.

\subsection{Film Characterization Techniques}

After films have been deposited, their properties must be measured. In order to characterize the thin film properties of interest, a large array of analytical tools can be brought to bear. By utilizing physical phenomena, it is possible to both measure macroscopic and microscopic properties of materials. Many of these techniques are used across the entire spectrum of scientific disciplines and industrial applications for research and quality assurance. In the following sections, different film characterization techniques will be outlined and discussed in some detail, but with the more focus on their use with 
regard to the research presented in this thesis. There is a large library of resources available, some of which will be referenced here, to learn more about any of these techniques for further study or interest. Therefore, much of the rigorous detail with regard to the theory and other applications are beyond the scope of this thesis and are left to those outside resources [38]. Briefly, X-Ray diffraction (XRD) and reflectometry (XRR) were used for measurement of film microstructure and thickness, respectively, while atomic force microscopy (AFM) was used for characterization of film surface morphology and scanning electron microscopy (SEM) was used for qualitative investigations of general surface quality as well as measurement of surface grain orientation via electron backscatter diffraction (EBSD). For magnetic property measurements, superconducting quantum interference device (SQUID) magnetometry was used for characterization of first penetration field and critical temperature in small coupon samples, while RF characterization methods were employed for cavity coatings.

\subsubsection{Diffraction Techniques}

Diffraction techniques include a wide slate of measurements which are possible in many areas of physics. Diffraction is an extremely useful physical phenomenon that allows for the exploration of properties of the physical world that are invisible to the human eye. Fundamentally, diffraction is a wave phenomenon that results from the interference of waves whether by slits or atomic lattices. Perhaps the earliest occurrences

of diffraction were in the field of optics many centuries ago, even as early as 984 AD, when 
early scientists were able to observe the bending of light through lenses and eventually the separation of the different wavelengths of light via prisms.

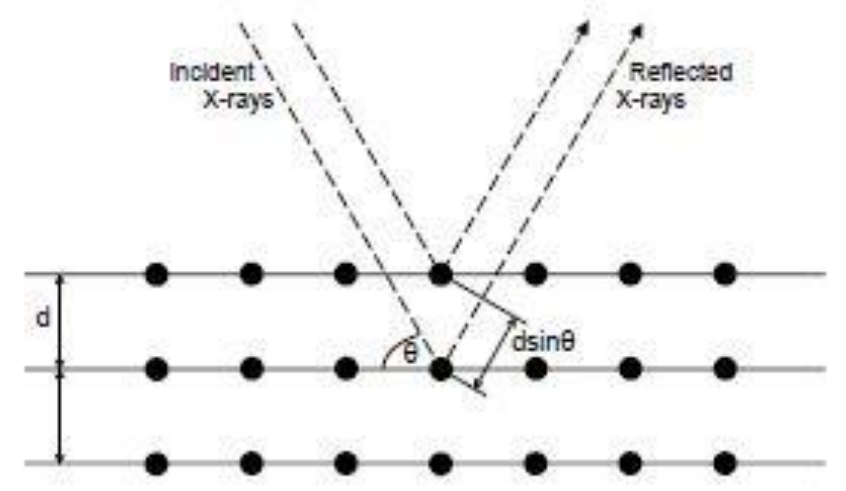

Figure 4.5: Schematic of X-ray diffraction from crystal lattice planes.

One form of diffraction that has major applications within the field of condensed matter physics is Bragg diffraction. For the research performed within this thesis, Bragg diffraction was used for investigation of the microstructure and thickness of films via XRD and XRR. Bragg diffraction, while fundamentally similar to all other forms of diffraction, is a result of the spacing and structure within solids. Instead of a grating or slit typically used for optical diffraction, the crystal lattice, that is, the periodic arrangement of the atoms, of the material serves as the mechanism by which diffraction takes place. As depicted in Figure 4.5, when an X-ray beam is incident upon the material, part of the beam is reflected at each interface between crystal lattice layers. Since there is periodic order throughout crystals, at a characteristic angle, dependent on the physical lattice dimensions, coherent interference patterns result from different atomic layers interfering with each other. This 
specific set of conditions required for diffraction are contained within what is known as the Bragg condition or Bragg's Law and are given by

$$
n \lambda=2 d \sin \theta
$$

where $n$ is the integer order of diffraction, lambda is the wavelength of the incident Xrays, $d$ is the interatomic spacing between atomic layers and $\theta$ is the incident angle of the X-ray beam with respect to the normal of the sample surface. Keeping in mind that the Bragg condition is only valid for $\lambda \leq 2 d$, one can see that it is possible using this technique to probe the microscopic structure of crystalline materials.

Generation of X-rays for this application in a laboratory environment typically occurs via electron ejection from a hot filament that when accelerated to a sufficient energy and incident upon an appropriate target, such as $\mathrm{Cu}$, atomic electrons can be ejected from the target material that then emit characteristic photons, $\mathrm{X}$-rays in this case, as valence electrons transition to fill the vacancies. The mono-energetic $\mathrm{X}$-rays are then focused into a quasi-parallel beam that is manipulated via collimation and filters and is then directed to illuminate the sample of interest. For all the work done in this thesis, a commercial PANalytical Empyrean 4-Circle Diffractometer was used for microstructure measurements. The measurements themselves utilized the $\mathrm{Cu} \mathrm{K}_{\mathrm{alpha}}$ emission X-rays with a wavelength of $0.15418 \mathrm{~nm}$, which is well below the diffraction limit.

While XRD is a common technique widely used in many fields such as criminal forensics, quality assurance, metallurgy and even pharmaceuticals, implementing it for thin films presents unique challenges. Typically, thin films produce a much weaker 
reflection than bulk crystalline materials due to their much smaller volume, imperfect microstructure and possible angular misalignments of the film to the substrate created during growth. These facts require great care and effort be put into the alignment and signal optimization of the beam, both incident and reflected, in order to effectively collect high quality data from thin film samples, especially when the crystal phases are not known beforehand. To overcome these challenges, modern X-ray diffractometers are equipped with strong alignment controls in the form of a four-circle goniometer, such as that shown in Figure 4.6. The four-circle goniometer provides the user with the ability to control multiple angles of rotation beyond the incident and reflected angle, as well as the vertical position, allowing for larger signal intensities to be collected from the sample.

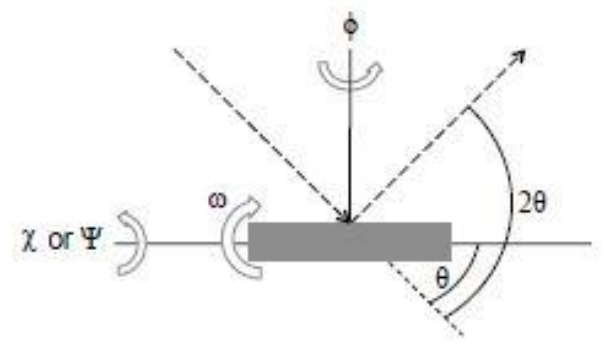

Figure 4.6: Schematic of a 4-Circle goniometer used in XRD system for film alignment.

For the work done in this thesis, XRD was utilized to gain information focusing on the out-of-plane lattice parameter, the average grain size depth of the crystallites, and the degree of long range order within the film, also called mosaicity. To obtain the previous data two types of XRD scans are used - high-angle theta-2theta scans and rocking scans or rocking curves, which are rotations in the omega angle with constant incident 
angle. From these scans the Bragg diffraction peaks can be collected and analyzed with respect to known material properties, typically obtained from standard powder diffraction reference files. The out-of-plane lattice parameter can then be obtained from the high-angle scans using the Bragg equation above while the crystallite grain size, $L$, is approximated via the Scherrer equation below

$$
L=\frac{K \lambda}{\beta \cos \theta},
$$

where $\mathrm{K}$ is a dimensionless geometric constant determined by crystal lattice symmetries and beta is the full-width half-maximum (FWHM) of the Gaussian Bragg peak. Finally, the mosaicity, or long-range order, can be obtained by determining the FWHM of the rocking curve mentioned above, where small FWHM represents a high degree of long-range order.

A useful variation of X-ray diffraction to thin films is X-ray Reflectometry (XRR). XRR is a special case of XRD where the X-ray beam is incident with the sample surface at a grazing angle. As the incident angle, theta, is varied slowly over a small range, the reflected intensity is recorded. When the films are of sufficient thickness, one can observe an interference pattern between the different atomic layers of the film and the interface of the film with the substrate. By taking this data and using algorithms to fit the data with specific models one can obtain information about the thickness, density and roughness of the film. However, alignment can be very difficult at times and great care should be taken during the model fitting since, if no reasonable estimations can be made, the models can be made to fit the data even if it is not representative of the physical system. For the research presented within this thesis, XRR was utilized to measure the thickness 
of growth calibration samples, used for determining growth rates of films at specific deposition parameters of interest and extrapolating to different times for other samples, which is of great importance to the multilayer work done since the field of first flux penetration enhancement is strongly thickness dependent.

So far, we have discussed diffraction of electromagnetic waves. However, another widely used diffraction method measures the results of electron diffraction at a material surface. One such technique used within this thesis is that of Electron Backscatter Diffraction (EBSD). EBSD operates on the principle that electrons incident upon the surface of a material collide with the atoms within the lattice, with some backscattering. When the electrons backscatter, some will emerge in accordance with the characteristic Bragg condition discussed earlier that relates the electron diffraction behavior to the crystalline structure of the material. In order to detect and measure these backscattered electrons, a phosphor screen is placed within the system to luminesce when electrons are incident and then a CCD camera is used to record the diffraction pattern, also known as a Kikuchi pattern. Once the pattern is recorded, measurements can be taken, or software used, to work backward and determine the crystalline structure of the material or film. EBSD is usually performed in tandem with a scanning electron microscope and, utilizing the precise capabilities of that technology, one can achieve high resolution of the crystal structure of a sample and even map the surface, false-colored for the different crystal orientations present, as can be seen in Figure 4.7. 

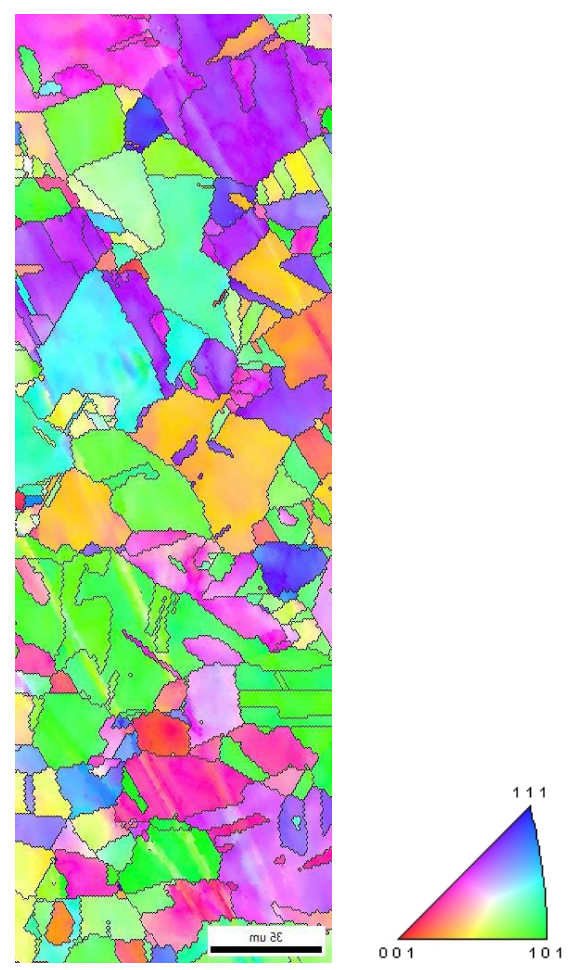

Figure 4.7: Example of EBSD data. In this case a polycrystalline $\mathrm{Cu}$ sample was measured and processed to form this image. OIM color map added as reference of crystal orientation.

\subsubsection{Microscopy}

This section will briefly describe the types of microscopy used for the work presented in this thesis: their advantages, disadvantages and the methods by which they work. Two forms of microscopies were used for the characterization of film surfaces on samples presented within this thesis. The two types used are: scanning electron microscopy (SEM) and atomic force microscopy (AFM). While both these techniques are contained under the microscopy heading, they are very different from each other and from the traditional optical microscopy most people are familiar with. The main benefit of microscopy techniques is the ability to create images of the film or material surface. Of 
the data presented within this thesis, SEM was mainly used for EBSD characterization discussed in the previous section and for quick qualitative checks of film surfaces, while AFM was employed to gain quantitative data of the film surface morphology for comparative studies between film properties and performance.

The first type of microscopy used was that of SEM. SEM machines are another widely used technology across many areas of research and industry and have progressed greatly over the years and today are capable of spatial resolution down to even $1 \mathrm{~nm}$ [2]. Fundamentally, SEM utilizes the physical phenomenon of secondary electron emission in order to create images of material surfaces. This is done by creating an electron beam in a high vacuum environment, focusing the beam via magnetic lenses and then directing it toward the sample surface through magnetic steering. Once electrons are incident upon the sample surface, there is a range of possibilities as to what may happen. Of importance are the percentage of electrons that stimulate secondary electron emission. Since these "true" secondary electrons are not affected by the film structure, as opposed to the backscattered electrons used for EBSD, they can be collected and turned into an image. One important note is that the secondary electrons are not material independent. This is due to the fact that the work function of different materials will alter the signal strength, the number or yield of secondary electrons. Therefore, materials with a lower work function or higher conductivity will appear brighter than those of lower work functions or conductivity. However, the secondary electrons are not so tied to the material type that the element may be determined via this interaction; for this purpose, SEM machines can utilize the photons emitted via electron collisions through a technique called energy 
dispersive X-ray spectroscopy (EDX, EDS), which gives elemental compositional information.

Some of the major advantages to SEM are due to the fact that the image brightness changes based upon the work function of the material and the geometry of the surface the electron beam is incident upon. As the topography of the sample surface changes, the angle which secondary electrons are emitted is altered and therefore the signal intensity from that location is lower than in a flat area. This offers the capability of resolving topographical features on sample surfaces. This gives very good qualitative information about the surface features present within the film such as grains, especially boundaries, roughness, defects and special structures possibly present. However, it is important to note that quantitative information cannot be gleaned from this process specifically. While it can give beautiful images, SEM should be reserved for general structural investigations and not for attempts to discern analytic information, though it is amazingly useful when paired with the analytical techniques possible using the hardware such as EBSD and EDS. In conjunction with the other techniques, SEM is a powerful scientific research tool.

The second form of microscopy used in this research was that AFM. AFM, in contrast to SEM, is a highly quantitative technique that not only gives detailed images of sample surfaces with very high resolution but also can be analyzed in order to obtain quantitative data from the sample. AFM works on the principle of interaction mediated via the Van Der Waals force. The Van Der Waals force is a weak force typically discussed in relation to interactions within liquids, such as water. In that case, the force is 
responsible for the surface tension and the relationship that molecules have with respect to each other. To take advantage of this force, an AFM contains a very precise scanning tip that is driven in a resonant mode by an outside force such as a laser or an electrical pulse. While the tip is being driven at resonance, the frequency of the tip is monitored via a laser reflection into a photodiode. Then, as the tip is lowered toward the sample surface, it comes into very close proximity with the surface. The proximity is in fact so close that the weak Van der Waals force between the tip and the sample affects the frequency with which the tip is resonating. Software can interpret this change in the resonant frequency of the tip into positional, or height, information since the frequency changes based upon the force, and therefore proximity, the tip experiences.

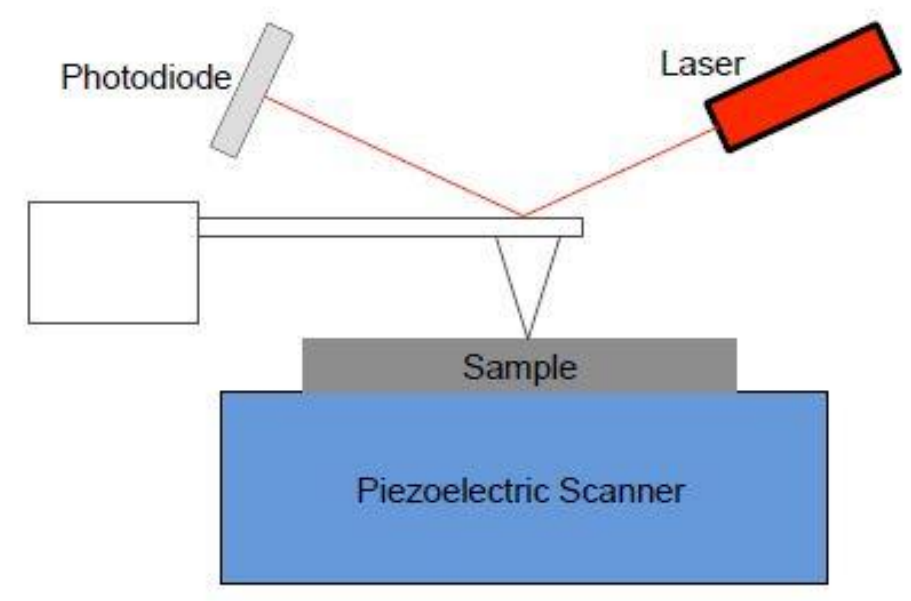

Figure 4.8: Simplified diagram of an AFM setup. Image credit: Doug Beringer.

Once the AFM system, depicted in Figure 4.8 has been calibrated appropriately, it is capable of moving the sample up and down via a piezoelectric pedestal and in the X-Y plane via a micro-stage. Using these tools, the AFM can scan the surface in the X-Y plane 
while moving the sample toward and away from the tip so as to get the height information while not, hopefully, coming into contact with the sample surface and causing damage. AFM has many uses as an analytic surface characterization tool. It is able to give quantitative data on the surface morphology of the sample as average or RMS surface roughness. It gives a detailed image of the sample surface with higher resolution than other techniques so microscopic structures, such as grains, can be clearly seen, as shown in an example AFM scan in Figure 4.9. Finally, AFM data can also be used for other analysis techniques such as power spectral density (PSD) analysis, which utilizes Fourier transforms of the data to look at the spatial frequency character of the roughness of the sample. While AFM is very useful as a tool, it can be a very time intensive technique with detailed calibrations and setup required for obtaining good images and it is very sensitive to external vibrations, even in air, which requires vibrational isolation of the system or the retaking of data after transient vibrations occur and blur images.

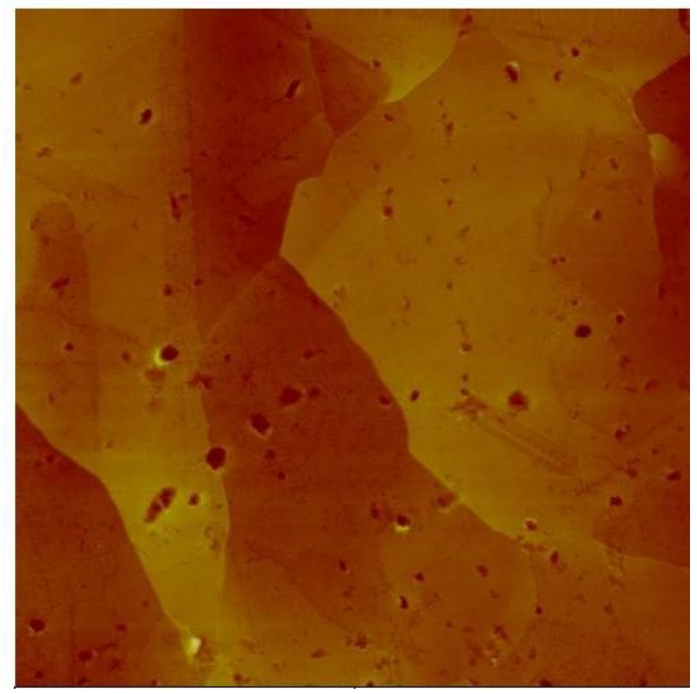

Figure 4.9: Example of surface image collected with an AFM. 


\subsubsection{Superconducting Property Measurements}

So far throughout this chapter, the topics discussed have focused on the creation of thin films through deposition techniques, which are the variables able to be changed in the experiment, and the characterization of the structural properties of the films as a result of the unique deposition methods and conditions used. In this section we will finally discuss the superconducting performance characterization techniques that are used in order to analyze and the properties of interest in this research. Within this research program, two main superconducting measurement techniques were used for the analysis of film performance and correlation of the results with the information obtained via the film structure characterization, and deposition conditions - superconducting quantum interference device (SQUID) magnetometry and RF cavity measurements.

In order to discuss and understand the technique of SQUID magnetometry, we must first refer back to the discussion in Chapter 2 on the theory of superconductivity. SQUIDs are extremely sensitive magnetic measuring devices that use systems called Josephson junctions. Josephson junctions are a unique technology that take advantage of the coherence length of superconductors. Coherence length, as discussed earlier, is the physical dimension over which the electrons maintain their interaction and form a bosonic Cooper pair. Using this knowledge, if two superconductors are connected via a weak link or barrier that is thinner than their coherence length, there exists a non-zero magnitude of the Cooper pair wavefunction present on each side of the barrier and therefore a probability for the Cooper pair to tunnel through the barrier, insulator or non- 
superconducting metal, and allow current to flow above a critical current Ic. An interesting and powerful feature of this is that, because the phenomenon is based upon Cooper pair tunneling, a SQUID is capable of detecting single flux quantum changes in magnetic fields; in fact, it is actually where magnetic flux quantization was originally shown. A loop with two such Josephson junctions in parallel, as shown in Figure 4.10, is known as a DC SQUID, while one Josephson junction is an RF SQUID. In order to measure magnetic field changes, a superconducting bias current is applied in the loop just below Ic which is split evenly between the two junctions. As the magnetic field changes across the loop, the current responds, dependent upon the magnitude of the change, which causes the phase between the two junctions to change and oscillate based upon the flux change in quanta. By counting the oscillations present, one is able to determine the total magnetic flux change experienced by the SQUID.

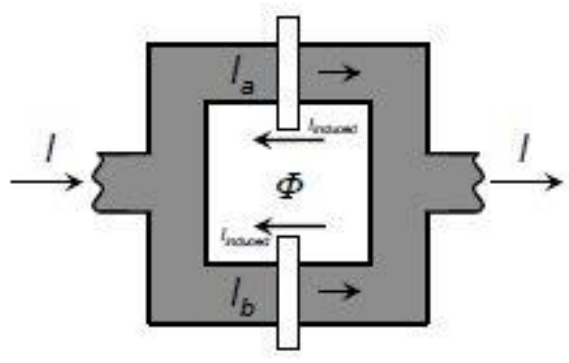

Figure 4.10: Schematic of a DC SQUID.

For this research, a commercial Quantum Design MPMS ${ }^{\circledR}$ (Magnetic Property Measurement System) XL DC SQUID magnetometer, which can apply fields from -7 to +7 Tesla, resolve magnetic flux changes on the order of femtotesla and maintain sample 
temperatures over the range 2-400K, was used to measure sample properties of interest. Specific properties of interest included the critical temperature $\left(T_{C}\right)$, lower critical field $\left(H_{C 1}\right)$, first flux penetration $\left(H_{f p}\right)$, and $A C$ susceptibility. In order to measure the desired values, the commercial system is equipped with a DC SQUID magnetometer, superconducting electromagnet, cryogenic temperature control unit, stepper motor for sample translation and a mount for holding the sample in the system and azimuthal rotation. The system utilizes liquid helium for cooling of the DC SQUID and the superconducting electromagnetic coils and liquid nitrogen as a preliminary cooling reservoir and thermal barrier to reduce liquid helium loss over time.

We provide a note here on the topic of intense debate within the field of superconducting thin films over the $\mathrm{H}_{\mathrm{C} 1}$ measurement. The determination of the measurement result as $\mathrm{H}_{\mathrm{C} 1}$ is hotly contested due to the practical setup in the DC SQUID system, which allows the magnetic field to be present on both sides of the sample and the edges thus bringing into question the true nature of the data collected and its relationship to $\mathrm{H}_{\mathrm{c1}}$. Perhaps the more general term to be used is that the field measured is the "field of first penetration" $\left(\mathrm{H}_{\mathrm{fp}}\right)$, however, throughout the rest of this thesis the value will be referred to as $\mathrm{H}_{\mathrm{c} 1}$ to agree with convention. Along with the controversy over the presence of the magnetic field surrounding the sample on all sides and around the edges, it is important to note that, even beyond this debate, all reported experimental $\mathrm{H}_{\mathrm{c1}}$ values using this kind of setup are necessarily underestimates of the true value. Due to the geometrical constraints present within the system, it is experimentally impossible to perfectly align the sample parallel to the applied field, beyond the presence of it on 
both sides. It can be shown as well that if a portion of the applied field is normal to the superconducting surface the result will be a lowering of the measured $\mathrm{H}_{\mathrm{c} 1}$.

Generally, magnetic SQUID measurements take place within the heart of the system where a sample no wider than $5 \mathrm{~mm}$, due to system geometry constraints, is moved through the DC SQUID pickup coils via a stepper motor in the presence of an applied magnetic field in the direction of motion. Schematically this can be seen in Figure 4.11. As the sample is moved through the pickup coils, the DC SQUID reacts to the changing magnetic flux caused by the sample and the system reads and interprets the voltage signals and converts them to data as a magnetic moment. This general procedure is present within all of the measurement types performed using the SQUID, except for the AC susceptibility, which allows for magnetic flux change measurements without translation through the coils.

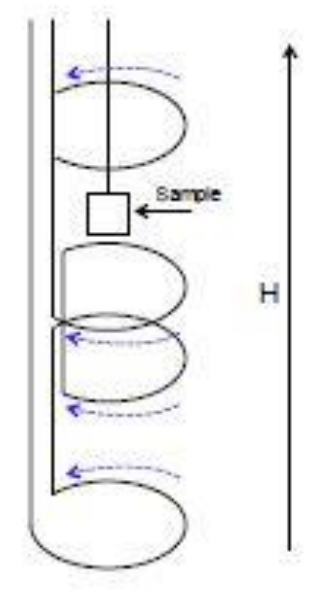

Figure 4.11: Example of the geometry within a SQUID pickup coils. Magnetization of sample is measured by signal picked up in Josephson junction as the sample is moved through pickup coils. 
The first property measured for any sample within the DC SQUID is the sample's critical temperature - the temperature at which the sample transitions from the normal conducting state above to the superconducting state. The measurement is performed by first cooling the sample down well below the expected $T_{c}$ where, once the temperature has stabilized, a small magnetic field of $10-25$ Oe is applied eliciting the Meissner effect from the sample and resulting in a negative magnetic moment. The sample is then warmed up slowly through discrete steps and the magnetic moment is measured at each step while the magnetic field is held constant. As the sample approaches $T_{c}$, the magnetic response approaches zero as can be seen in Figure 4.12. While there are multiple conventions used by different fields for the determination of $T_{c}$ from this data, for the purposes of results presented within this thesis, the $T_{C}$ is defined as the point at which the slope of the M-vs-T graph changes from concave upward to concave downward as determined by a second derivative analysis of the data.

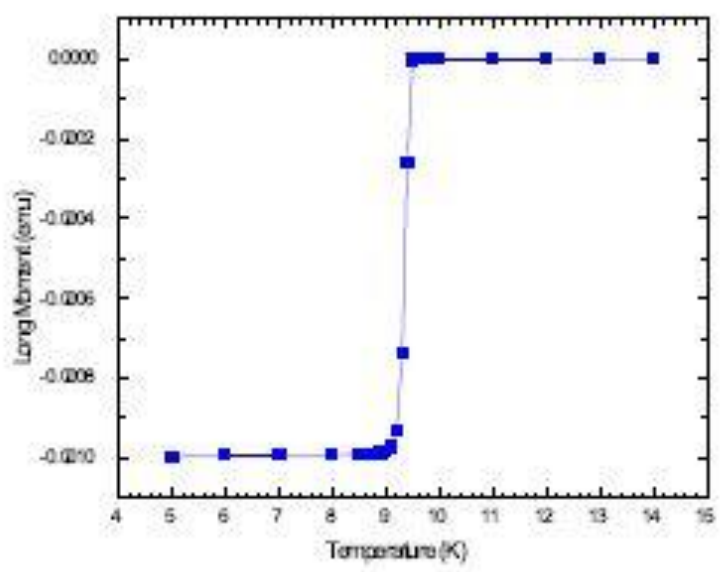

Figure 4.12: Example critical temperature data graph of superconducting sample magnetization measured through its critical transition temperature. 
While the measurement and determination of the sample critical temperature are straightforward with little ambiguity, the measurement of a superconducting sample's lower critical field, while straightforward in collection, is much more hotly debated, as mentioned earlier. Two methods were employed for the collection of critical field data in this research. The following describes schematically the measurement process; it then proceeds from this point iteratively. First, the sample is cooled down in zero magnetic field to a standard temperature below $\mathrm{T}_{\mathrm{c}}$, typically $5 \mathrm{~K}$, which is maintained for all superconducting measurements. The sample then undergoes a zero-field background measurement. Then, a magnetic field is applied and the sample is measured a second time with the field held constant. Finally, the field is removed and the sample is measured at zero field a second time to record any trapped flux remaining in the sample. After this point, the sample is warmed above $T_{C}$ to remove any magnetic history and the procedure is repeated for a new, typically higher, applied magnetic field.

Using this iterative method, a wealth of data is collected that allow the determination of $\mathrm{H}_{\mathrm{C1}}$ through two analysis methods. For both methods the background data collected in the initial scan is subtracted from the other two scan data. From the second scan data one can create the familiar M-H curve shown in Figure 4.13. Due to the Meissner effect of superconducting films, as the magnetic field is increased, the sample creates a magnetic field equal and opposite in a linear fashion up to a point; this linear relationship is referred to as the Meissner slope. The last point at which the magnetic moment of the sample remains linear in response to the applied field is referred to as $\mathrm{H}_{\mathrm{c1}}$. The second method of $\mathrm{H}_{\mathrm{C} 1}$ determination lies in analyzing the data collected in the third 
scan described above known as the trapped flux method. After subtracting the background data from this scan, one can plot the data. Since no trapped flux, and therefore no magnetic moment, should remain in a film after the applied field has been removed, the measured magnetic moment should remain zero for this measurement. As the applied magnetic field is increased to the point of reaching $\mathrm{H}_{\mathrm{C} 1}$, some portion of the sample traps flux as vortices becomes normal conducting within the vortex core. When the field is then removed, a small amount of the applied field is trapped in the sample giving it a non-zero magnetic moment. Therefore, to determine $\mathrm{H}_{\mathrm{c} 1}$, the data can be plotted and the last data point collected that does not deviate from zero is defined as $\mathrm{H}_{\mathrm{c1}}$. For the work done in this thesis, all the reported $\mathrm{H}_{\mathrm{C} 1}$ values were determined via the deviation from Meissner slope method with the trapped flux measurement used as a secondary confirmation of the results.

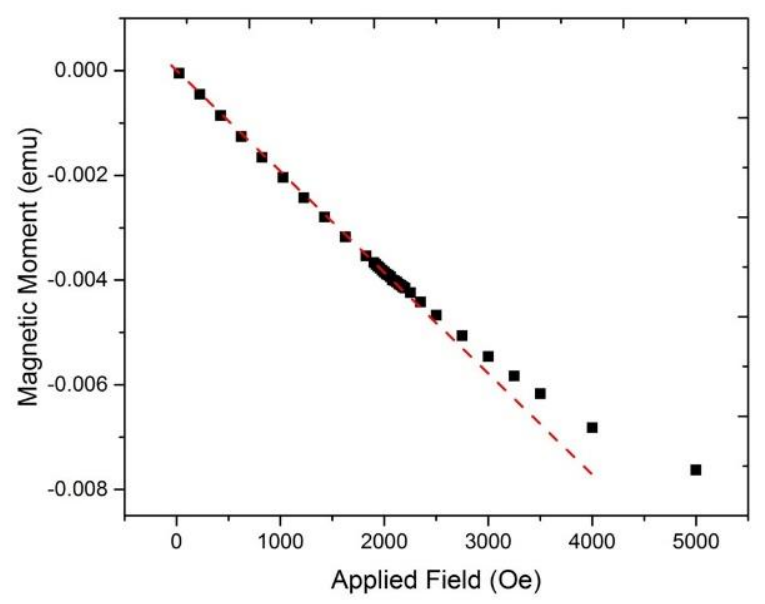

Figure 4.13: Example graph of $\mathrm{M}$ vs $\mathrm{H}$ curve collected via SQUID magnetometry and plotted with a line showing the deviation from the linear Meissner slope indicating flux penetration. 
RF measurements are the final and most important measurement of any film for the desired application of this project. Since all films were studied with the ultimate goal of application to SRF accelerator cavities, RF performance measurements are key to advancing the work. While there are systems around the world capable of performing RF measurements on relatively small samples [39] [40], typically 2-4" in diameter, they are relatively few in number, have long queues for getting samples measured, can be expensive and are not necessarily indicative of cavity coating performance since cavities are necessarily different in deposition conditions for a wide array of reasons. To this end, the RF data presented in this thesis are entirely made up of RF cavity measurements performed at JLab in their Vertical Test Area (VTA).

RF cavity measurements are conducted at JLab within large cryogenic dewars with radiation detection and shielding. The cavities are mounted on large inserts that maintain the cavities under high vacuum with RF power fed into the cavity via input probes; the forward and the transmitted power is collected via pickup probes. These probes couple to the cavity field profile where the input probe is coupled appropriately for efficient transfer of power from the RF source to the cavity, while the pickup probe is typically weakly coupled with an external $Q$ much higher than the cavity $\left(Q_{0}\right)$ so as to only pick off a small pfraction of the RF power in the cavity and not complicate the measurement. It is important in this step to set the probes based upon the expected $Q_{0}$ of the cavity itself in the superconducting state for the most efficient and accurate data with low error. However, this can be difficult for R\&D purposes where the cavity $Q_{0}$ is by definition not explicitly known beforehand and therefore must be approximated. 
Typical measurement techniques employed for RF testing are: stored energy, decay and calorimetric methods. Each method has its benefits and drawbacks and which technique is used typically depends on the specific conditions for each test. Using these methods, one is able to measure the RF response of the cavity and thereby determine multiple values of interest. Some properties of interest are: transition temperature, accelerating gradient and quality factor. Typically, the main graph of interest for any cavity, especially thin film cavities, is that of the quality factor as a function of the applied field or accelerating gradient. Other values of interest are the BCS surface resistance and the residual resistance parts of the quality factor. This is obtained by holding the accelerating gradient constant, typically low at a few $\mathrm{MV} / \mathrm{m}$, and changing the temperature while measuring the surface resistance. The resulting $R_{s}$ vs $T$ curve can be fit and the BCS model parameters extracted to calculate the BCS surface resistance and the leftover residual resistance. This also gives information on residual resistance of the cavity.

While RF measurements were heavily employed for the $\mathrm{Nb} / \mathrm{Cu}$ work done in this thesis, the details of such methods are slightly beyond the scope of this dissertation with a discussion of RF electromagnetism needed. Therefore, the interested reader is referred to an extensive library of resources on the topic including books on RF measurement, cavity testing and journal articles covering contemporary cavity testing. [24] 


\section{CHAPTER 5}

\section{NbN \& NbTiN Thin Films for SIS Multilayers}

The next two chapters are dedicated to presenting experimental results from studies performed for $\mathrm{Nb}$ thin film coated SRF cavities using HiPIMS and early tests of the multilayer thin film scheme discussed previously for the enhancement of the penetration field for SRF cavities. This chapter will be dedicated to the work done on coating small coupon samples and SRF cavities with $\mathrm{Nb}$ thin films using HiPIMS; specifically, results of correlative studies will be presented linking film structure and morphology to the deposition parameters employed for the films. After, results from $\mathrm{Nb}$-coated SRF cavities will be presented and discussed with an emphasis on challenges specific to cavity preparation, handling and deposition.

In this chapter, experimental results will be presented from the research done on depositing $\mathrm{NbN}$ and $\mathrm{NbTiN}$ films. The films were created to test the thickness dependence of the $\mathrm{H}_{\mathrm{C1}}$ enhancement and the applicability of the SIS multilayer scheme proposed by Gurevich et al. [11] to SRF accelerating structures. Work on multilayer films for SRF cavities has been a longstanding research interest in Dr. Lukaszew's research group at William \& Mary with many publications over the years. The specific work presented here is the culmination of years of work and collaboration presented at conferences and published in a variety of peer-reviewed journals [41] [42] [43] [44] [45] [46] [47] [48] [49]. 


\subsection{Experimental Setup}

All the $\mathrm{NbN}$ and $\mathrm{NbTiN}$ films and multilayers were prepared at William and Mary in the bell-jar system, outlined in Chapter 4, on commercially-obtained single crystal $\mathrm{MgO}(100)$ substrates using DC magnetron reactive sputtering with a fixed target-tosubstrate distance of $13.5 \mathrm{~cm}$. Prior to film growth, the substrates were cleaned in an ultrasonic bath of alternating acetone and methanol and annealed at $600^{\circ} \mathrm{C}$ for one hour under vacuum; this temperature was subsequently maintained during deposition. After growth, the structural properties of the samples were determined via XRD and the DC superconducting properties were measured via SQUID magnetometry. Additionally, some NbTiN samples shown here were created at JLab in their UHV deposition system by Dr. Anne-Marie Valente-Feliciano on different substrates; the analysis of their structure and superconducting properties was performed alongside the samples created at William \& Mary. A crucial factor when looking at superconducting alloy films, which will be discussed in the following sections, is the relationship between film stoichiometry and performance. As we will see, this is true for both the binary system of $\mathrm{NbN}$ and, perhaps even more crucially, for the ternary $\mathrm{NbTiN}$ material.

\subsection{NbN Thin Films}

$\mathrm{NbN}$ is a promising candidate material for the proposed multilayer scheme with it's $\mathrm{H}_{\mathrm{c} 1}$ of $200 \mathrm{Oe}$ and $\mathrm{T}_{\mathrm{c}}$ of $16.2 \mathrm{~K}$, nearly twice that of Nb. NbN films were prepared using the above described system with a 2 " $\mathrm{Nb}$ target of $99.95 \%$ purity in an Ar and $\mathrm{N}_{2}$ gas mixture. The $\mathrm{NbN}$ films were first calibrated to achieve the most bulk-like properties 
consistent with earlier studies done within the group. Once the optimal deposition parameters were found, a thickness series was created to observe the relationship of the films $\mathrm{H}_{\mathrm{C} 1}$ and its thickness. To that end, the films were deposited at a power of $60 \mathrm{~W}$ with an Ar pressure of 4.5 mTorr and $\mathrm{N}_{2}$ partial pressure of 0.05 mTorr. Some films were also topped with a thin gold capping layer to observe the effect of a protected and smoother surface on the DC superconducting properties of the films. For a well-formed, more indepth discussion of the correlation of the stoichiometry and microstructural properties to the superconducting properties of $\mathrm{NbN}$ the reader is referred to the following journal article which compiles years of work done on NbN at W\&M [41] [45] [46] [47] [49].

Table 5.1: Properties of NbN samples grown and characterized for this study.

\begin{tabular}{|l|l|l|l|l|}
\hline Sample & Thickness $(\mathrm{nm})$ & Lattice Parameter $(\AA)$ & $\mathrm{T}_{\mathrm{C}}(\mathrm{K})$ & $\mathrm{H}_{\mathrm{C1}}(\mathrm{Oe})$ \\
\hline A & 36 & 4.34925 & 10.98 & 76 \\
\hline B & 120 & 4.35677 & 9.6 & 140 \\
\hline C & 85 & 4.35776 & 9.6 & 160 \\
\hline D & 121 & 4.36112 & 11.34 & 1600 \\
\hline E & 214 & 4.37392 & 13.84 & 470 \\
\hline F & 85 & 4.37913 & 13.72 & 500 \\
\hline G & 378 & 4.38238 & 10 & 230 \\
\hline H & 150 & 4.3854 & 14.55 & 270 \\
\hline I & 100 & 4.38737 & 14.24 & 350 \\
\hline J & 120 & 4.38795 & 14.75 & 950 \\
\hline K & 80 & 4.38814 & 14.63 & 1000 \\
\hline L & 60 & 4.39078 & 14.55 & 700 \\
\hline Bulk & - & 4.395 & 16.2 & 200 \\
\hline
\end{tabular}




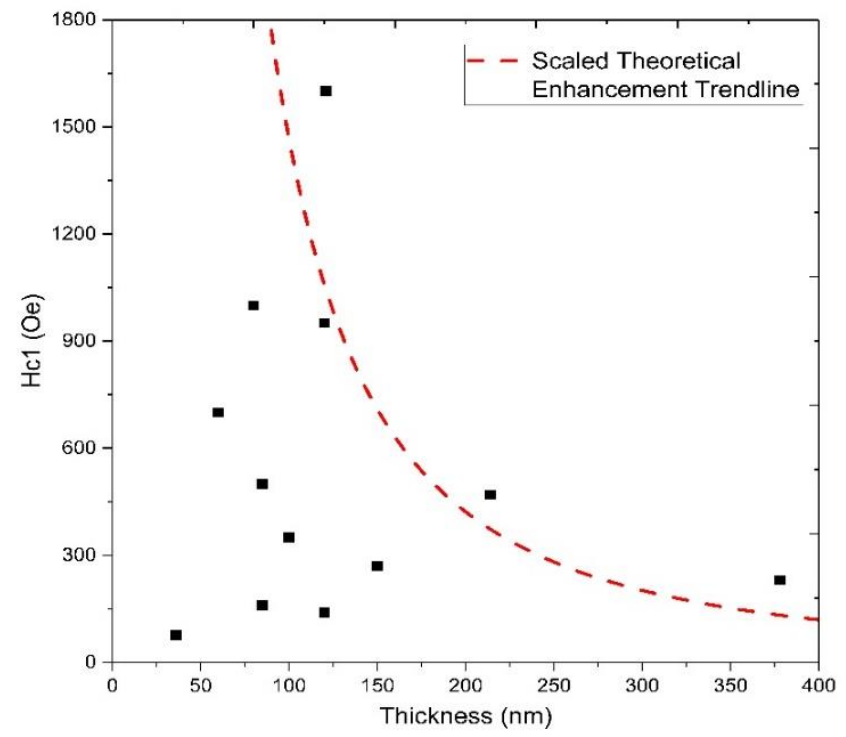

Figure 5.1: Plot of the lower critical field $\left(\mathrm{H}_{\mathrm{c1}}\right)$, or field of first flux penetration, versus thickness testing the proposed enhancement. A scaled theoretical enhancement trendline has been overlaid as a guide for the eye.

Table 5.1 lists some of the properties of the NbN films grown, including thickness, lattice parameter, $\mathrm{T}_{\mathrm{c}}$ and $\mathrm{H}_{\mathrm{c} 1}$. Most importantly, if the $\mathrm{H}_{\mathrm{c} 1}$ is plotted against film thickness, shown in Figure 5.1, it can be seen that $\mathrm{H}_{\mathrm{c} 1}$ does indeed increase above the bulk $\mathrm{NbN}$ value of 200 Oe for films thinner than their London penetration depth. Also, if the lattice parameter is plotted against $\mathrm{H}_{\mathrm{C} 1}$ and $\mathrm{T}_{\mathrm{c}}$, one can observe that the samples that performed the best - with the largest $\mathrm{H}_{\mathrm{C} 1}$ increase and highest $\mathrm{T}_{\mathrm{C}}$ values - also exhibited the most bulk-like microstructure. While all the samples shown here did not receive an enhancement on the theoretically-predicted level, it is useful to also remind the reader that (1) theory assumes perfect bulk-like behavior while thin films fundamentally are different in structure and behavior than that of their bulk counterparts and (2) all of the $H_{C 1}$ values listed are necessarily underestimates, as discussed in the SQUID experimental section of Chapter 4. 


\subsubsection{NbN-Based Trilayers}

In order to assess the viability of the multilayer theory for SRF cavity applications, we must expand beyond simple single layer coupon samples. The next logical step in this process is to create multilayer samples. With this need in mind, we first set out to create adequate single-layer $\mathrm{NbN}$ films, presented in the previous section, since this material is the simplest to transition into the multi-layer scheme. Once films of adequate characteristics were demonstrated, we undertook the growth of $\mathrm{Nb} / \mathrm{MgO} / \mathrm{NbN}$ trilayered films. The trilayers were grown by reactive, regular and pulsed DCMS, for the $\mathrm{NbN}, \mathrm{Nb}$ and $\mathrm{MgO}$ layers respectively, on 2" $\mathrm{MgO}(001)$ substrates and consisted of: a very thin (< $5 \mathrm{~nm}$ ) MgO seed layer over the crystalline substrate in order to improve surface morphology, based on our previous work, followed by a base $250 \mathrm{~nm}$ layer of $\mathrm{Nb}$, a $15 \mathrm{~nm}$ MgO layer, and finally an $85 \mathrm{~nm} \mathrm{NbN}$ layer. The NbN layer was grown under the same deposition conditions presented in the above NbN section. The choice of thickness for the $\mathrm{Nb}$ layer was done to preserve adequate surface roughness for subsequent growth, while the choice of $\mathrm{NbN}$ thickness was below the London penetration depth and based on our previous work.

When undergoing this growth, of utmost concern is the quality of the interfaces and achieving the correct phase correlated to optimal superconducting properties. This arises from the fact that, while it is possible to achieve good epitaxy between the $\mathrm{NbN}$ and a crystalline $\mathrm{MgO}(001)$ substrate, strain can affect the surface morphology, thus affecting subsequent epitaxial growth through multiple interlayers. Figure 5.2 shows XRD 
scans for a the $\mathrm{NbN}$-based trilayers, where we observe that the appropriate $\delta-\mathrm{NbN}(002)$ phase with the absence of any other $\mathrm{NbN}$ phases was achieved. The corresponding lattice parameters for the trilayer films were around $4.38 \AA$, i.e. within bulk value range. Also, the grain sizes varied from $26-27 \mathrm{~nm}$. We track this characteristic of film microstructure, because it is known that grain boundaries are responsible for degraded superconducting thin films compared to bulk [25].

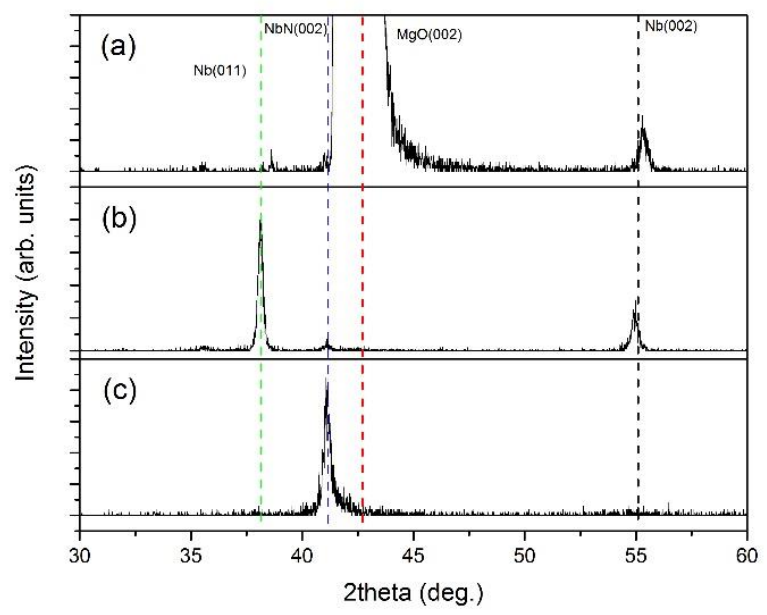

Figure 5.2: XRD scans for NbN Trilayer samples a) 092314 b) 100114 and c) 100714 . All exhibit only the $\mathrm{NbN}(002)$ phase showing good epitaxy with top NbN layer and underlying substrate. Note, all scans were optimized on the $\mathrm{NbN}(002)$ reflection and varying misalignments of the layers leads to differing intensities of reflections between samples. Highest performing film exhibited predominantly $\mathrm{Nb}(002)$ texturing, confirming previous results found by group. [50]

We note that the XRD scan shows that, while one of the samples exhibits only the $\mathrm{Nb}$ (002) phase for the $\mathrm{Nb}$ layer, the other sample exhibited $\mathrm{Nb}(011)$ and $\mathrm{Nb}(002)$ texturing. This is important since it has been shown previously [5] that $\mathrm{Nb}$ samples with (011) texturing exhibit poorer superconducting performance than those with only (002) texturing due to grain boundaries. 


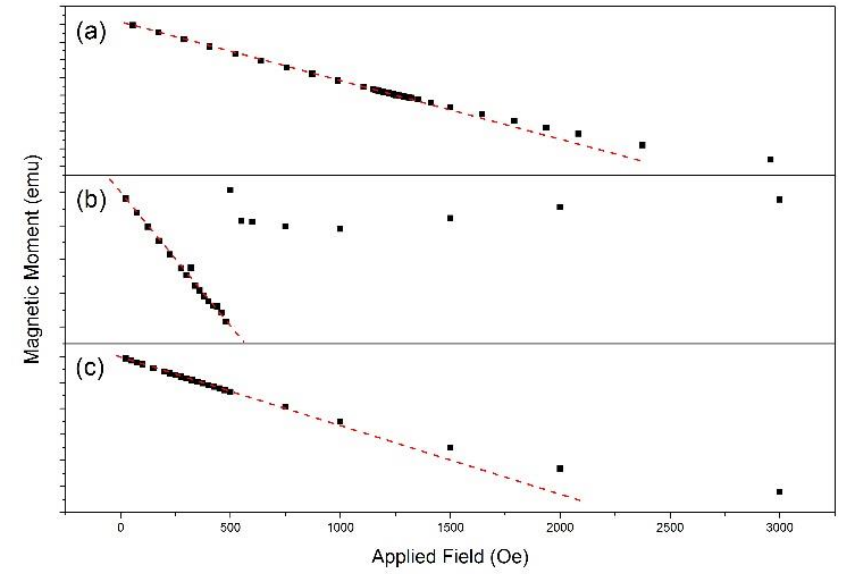

Figure 5.3: SQUID magnetometry data for $\mathrm{Hc1}$ determination of NbN-based trilayer sample a) 092314 with an Hc1 of 2100 Oe b) 100714 with an Hc1 of $\sim 480$ Oe and c) 100114 with an Hc1 of 750 Oe. The data was acquired using the Bohmer method referenced and Hc1 was determined as the point before the deviation from the Meissner slope.

All of the trilayers exhibited good DC superconducting properties with $T_{c}$ values around $13 \mathrm{~K}$, again reaffirming the XRD result of the adequate delta phase of $\mathrm{NbN}$. The samples also exhibited large enhancements in the $\mathrm{H}_{\mathrm{c} 1}$ values compared to bulk $\mathrm{NbN}$ and, as shown in Figure 5.3, the best sample had an approximate $\mathrm{H}_{\mathrm{C} 1}$ value of over 2000 Oe, over 10 times larger than bulk $\mathrm{NbN}$ value and an improvement over our earlier results. These results showcase the great promise of the multilayer approach while underscoring the importance of adequate control of thin film stoichiometry, microstructure and surface parameters.

\subsection{NbTiN Thin Films}

A set of NbTiN films were grown using a single 2" 70/30 (wt. \%) NbTi target in an Ar and $\mathrm{N}_{2}$ gas mixture with a fixed target to substrate distance of $13.5 \mathrm{~cm}$. The growth 
parameters of the NbTiN films were optimized by varying the total pressure, mass flow rate, $\mathrm{N}_{2}$ partial pressure, and substrate temperature while comparing the microstructure of the resulting films aiming at the most bulk-like properties. To this end, films were grown using $2.5 \% \mathrm{~N}_{2}$ partial pressure, a total pressure of $4.1 \mathrm{mTorr}$, and a substrate temperature of $600^{\circ} \mathrm{C}$, since it has been shown that higher temperature growth leads to films with higher Tc values [51]. A second set of films were grown in a similar setup at JLab by Dr. Anne-Marie Valente-Feliciano using DC magnetron sputtering on bulk Nb, AIN and crystalline $\mathrm{MgO}$ substrates with an $80 / 20$ (wt. \%) $\mathrm{NbTi}$ target, a fixed target to substrate distance of $8 \mathrm{~cm}$, and with a thin AIN interlayer grown prior to NbTiN deposition. The films used in these experiments varied in thickness from $50 \mathrm{~nm}$ to $2 \mu \mathrm{m}$. Table 5.2 below lists some of the deposition parameters and physical properties of the NbTiN films used in this study.

Table 5.2: Table of Deposition Conditions and Relevant Physical Properties of NbTiN Samples Used in this Study.

\begin{tabular}{|c|c|c|c|c|c|c|}
\hline Sample & Substrate & $\begin{array}{c}\text { Target Stoichiometry } \\
(\mathrm{Nb} / \mathrm{Ti} \% \mathrm{wt})\end{array}$ & \% Nitrogen & $\begin{array}{c}\text { Lattice } \\
\text { Parameter }(\AA)\end{array}$ & $\begin{array}{c}\mathrm{H}_{\mathrm{c} 1} \\
(\mathrm{Oe})\end{array}$ & $\mathrm{T}_{\mathrm{c}}(\mathrm{K})$ \\
\hline $\mathrm{a}$ & $\mathrm{MgO}(001)$ & $70 / 30$ & 2.5 & 4.318 & 140 & 12 \\
\hline $\mathrm{b}$ & $\mathrm{MgO}(001)$ & $70 / 30$ & 2.5 & 4.324 & 320 & 13.1 \\
\hline $\mathrm{c}$ & $\mathrm{MgO}(001)$ & $70 / 30$ & 2.5 & 4.327 & 140 & 12.9 \\
\hline $\mathrm{d}$ & $\mathrm{MgO}(001)$ & $70 / 30$ & 2.5 & 4.305 & 300 & 6.8 \\
\hline $\mathrm{e}$ & $\mathrm{MgO}(001)$ & $70 / 30$ & 2.5 & 4.324 & 600 & 13.5 \\
\hline $\mathrm{f}$ & $\mathrm{Nb}($ bulk) & $80 / 20$ & 23 & 4.353 & 350 & 15.97 \\
\hline $\mathrm{g}$ & $\mathrm{Nb}(\mathrm{bulk})$ & $80 / 20$ & 23 & 4.343 & 300 & 16.57 \\
\hline $\mathrm{h}$ & $\mathrm{MgO}(001)$ & $80 / 20$ & 23 & 4.359 & $\mathrm{NA}$ & 16.23 \\
\hline $\mathrm{i}$ & $\mathrm{MgO}(001)$ & $80 / 20$ & 23 & 4.356 & $\mathrm{NA}$ & 15.2 \\
\hline $\mathrm{j}$ & $\mathrm{MgO}(001)$ & $80 / 20$ & 23 & 4.360 & 1000 & 16.05 \\
\hline $\mathrm{k}$ & $\mathrm{MgO}(001)$ & $80 / 20$ & 23 & 4.351 & 2000 & 15 \\
\hline $\mathrm{l}$ & $\mathrm{MgO}(001)$ & $80 / 20$ & 23 & 4.346 & 1350 & 16.7 \\
\hline $\mathrm{m}$ & $\mathrm{AlN}(\mathrm{cer})$ & $80 / 20$ & & & & \\
\hline
\end{tabular}


We note that the NbTiN system has many possible phases; hence, the resulting film may have different properties if any of these phases are also present in addition to the desired superconducting B1 phase. During deposition, phases present in the films can be tailored by altering parameters such as pressure. For this research, it is of utmost importance to create films in the optimal superconducting B1 phase of NbTiN. Thus, a parameter of particular interest was the nitrogen partial pressure. In order to optimize the nitrogen partial pressure, a systematic study was carried out changing only the $\mathrm{N}_{2}$ partial pressure while holding other variables constant during growth. The films were then analyzed using XRD to determine the resulting lattice parameters, grain size, and mosaicity.

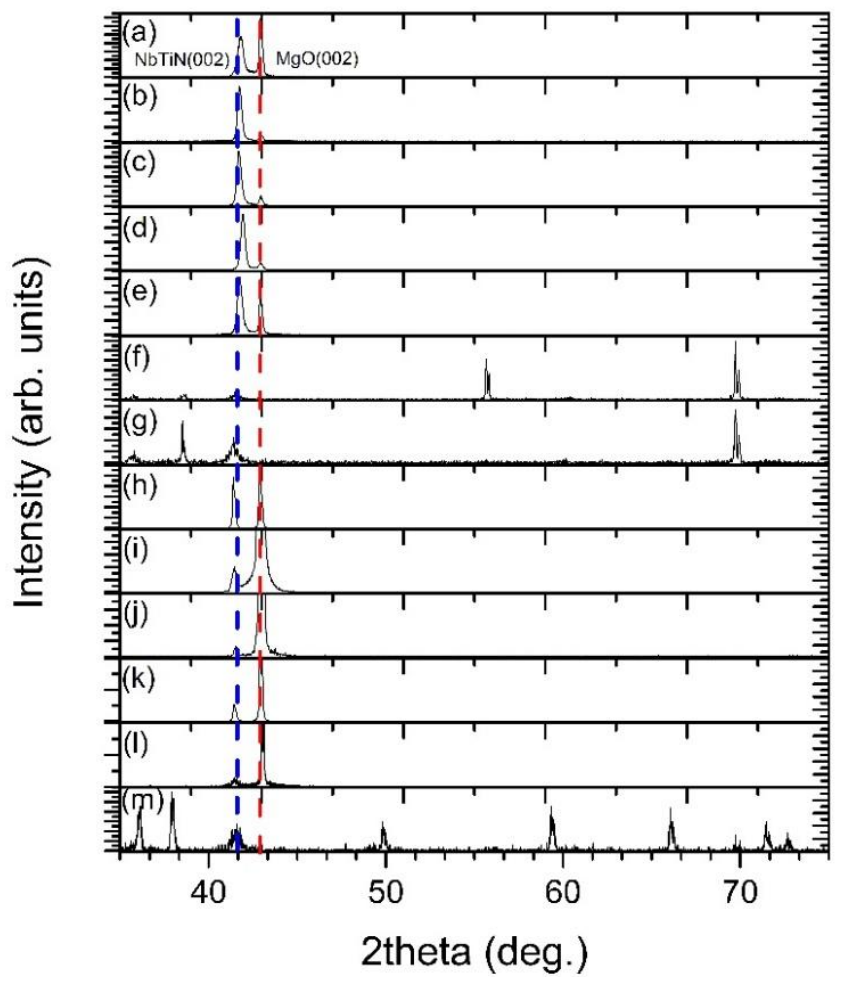

Figure 5.4: XRD scans from all of the NbTiN samples measured for this study. The letter labels correspond to those in Table 1. Samples (f)-(m), made with the 80/20 target, exhibit a clear shift to lower angles corresponding to a larger lattice parameter than those made with the 70/30 composition target. The extra peaks present in samples in (f) \& (g) correspond to the multiple phases of $\mathrm{Nb}$ present in the bulk $\mathrm{Nb}$ substrate for those samples while those in sample (m) result from the ceramic AIN substrate used. 
From the XRD scans in Figure 5.4, our samples exhibit reflections from the crystalline substrates and $\mathrm{NbTiN}(002)$ reflections corresponding to the B1 phase, showing clear epitaxial growth of the $\mathrm{NbTiN}$ on the $\mathrm{MgO}(001)$ substrates. No other phases of $\mathrm{NbTiN}$ are observable in the range from 20 to $80^{\circ}$ in our samples, leading to the conclusion that they exhibit only the optimal superconducting B1 phase. The grain sizes of our samples were obtained from FWHM of the NbTiN (002) XRD peak and the Scherrer equation. The grain sizes varied between 28 and $40 \mathrm{~nm}$, while the film with the highest $\mathrm{H}_{\mathrm{C1}}$ value had a grain size of approximately $31 \mathrm{~nm}$.

Adequate stoichiometry, to achieve the appropriate superconducting phase in a ternary compound, is very important [52]. From our measurements, the lattice parameter is an indicator of film stoichiometry. NbTiN is a ternary alloy and as such, the actual composition of a thin film depends on adequate nitrogen partial pressure as well as target composition when using a composite NbTi target. Primarily, the stoichiometry of a particular film determines its lattice parameter. Once films with close to bulk composition and properties have been achieved, as evident by the lattice parameters, one can begin to vary the thickness in order to improve $\mathrm{H}_{\mathrm{c} 1}$. At this stage, effects such as grain size, surface roughness, and strain will start to play a secondary role in determining the film properties. In addition, some variations are also expected, in both the grain sizes and lattice parameter, due to the strain from lattice mismatch and grain on grain growth of the films with the various substrates. 
We note that in order to achieve films with the desired composition, it is possible to work under deposition conditions that are near the edge for the formation of unstable phases, i.e., in an unstable reactive deposition regime. Thus, for this study, two targets of differing stoichiometry were used to investigate the dependence of the resulting films' superconducting properties on the growth stability, as evidenced by the lattice parameter of the resulting films. To this end, two different target compositions, a 70/30 (wt. \%) NbTi target operating in the unstable reactive deposition and an 80/20 (wt. \%) target were tested. We note that one of the difficulties associated with controlling the composition of the resulting films is the inherent difference in the relative sputtering rates for $\mathrm{NbN}$ and TiN. Hence, the final composition will not only be dependent on the nitrogen partial pressure and power, but also on the composition of the alloy target being used.

The films grown using a 70/30 (wt. \%) NbTi target resulted in B-phase films with lattice parameters between 4.30 and $4.327 \AA$ and the film with the largest $H_{C 1}$ value had a lattice parameter of $4.32 \AA$. While B-phase films grown using the $80 / 20$ (wt. \%) target exhibited somewhat larger lattice parameters, between 4.34 and $4.36 \AA$ with the film with the largest $\mathrm{H}_{\mathrm{C} 1}$ value having a lattice parameter of $4.35 \AA$. We note here the relatively low value and large variance in the lattice parameter of the films grown using the 70/30 target compared to the samples grown with via the $80 / 20$ target, indicating process control instabilities when using the 70/30 target. This topic will be discussed further in the conclusion.

The superconducting properties of bulk NbTiN are difficult to find in the literature due to the fact that NbTiN is not a natural-occurring compound. Therefore, unlike other 
materials, "bulk" values are not readily available. One can only report on the optimal values observed for experimentally achieved NbTiN. In this regard, it has been reported that $\mathrm{NbTiN}$ has a highest observed $\mathrm{T}_{\mathrm{C}}$ around $18 \mathrm{~K}$ [53]. When comparing across our samples in Table 5.2, we note that the samples grown using the $80 / 20$ target had overall better DC superconducting properties than the $70 / 30$ counterparts. The $70 / 30$ samples exhibited $T_{C}$ values between 12 and $14 \mathrm{~K}$ while the 80/20 samples achieved $\mathrm{T}_{\mathrm{C}}$ values up to $17 \mathrm{~K}$. The $\mathrm{H}_{\mathrm{C} 1}$ values in all samples were determined from SQUID DC measurements in the parallel-field to the film surface geometry, which were carried out minimizing systematic errors following a standard method described in Chapter 4 [54].

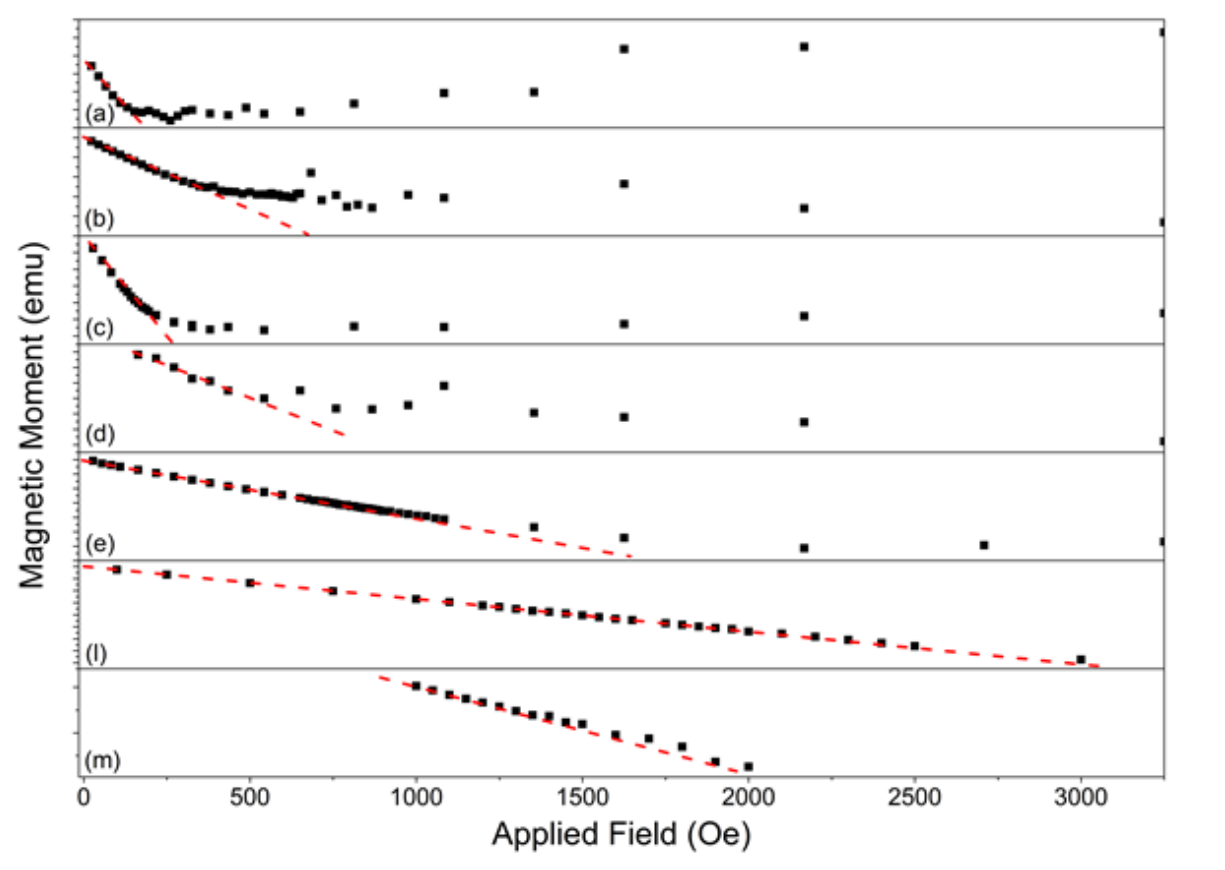

Figure 5.5: SQUID magnetometry data for select NbTiN samples with $\mathrm{H}_{\mathrm{c} 1}$ values of (a) 140 Oe (b) 320 Oe (c) 180 Oe (d) 500 Oe (e) 830 Oe (I) 2000 Oe and (m) 1350 Oe. Data was obtained and analyzed using the Bohmer method mentioned above. All samples exhibited purely the $\mathrm{NbTiN}$ (002) phase and those with larger lattice parameters similarly had higher $\mathrm{H}_{\mathrm{c} 1}$ values. The letter labels correspond to those in Table 5.2. 
As can be seen in Table 5.2 and Figure 5.5, the highest $\mathrm{H}_{\mathrm{c} 1}$ observed in the films grown using the 70/30 target was around 830 Oe while the highest for the $80 / 20$ films was on the order of 2000 Oe. However, it is also necessary to again note here that all of the $\mathrm{H}_{\mathrm{c} 1}$ values reported are necessarily underestimates of the "true" value due to geometric constraints within the measurement system, i.e., the experimental difficulty in ensuring that the sample surface is absolutely parallel to the applied DC field. The $\mathrm{H}_{\mathrm{C} 1}$ enhancement quoted is only valid in absolutely parallel DC field geometry, and any component of the applied magnetic field perpendicular to the sample surface will necessarily result in a reduction in the observed $\mathrm{H}_{\mathrm{c} 1}$ value due to early onset magnetic flux penetration.

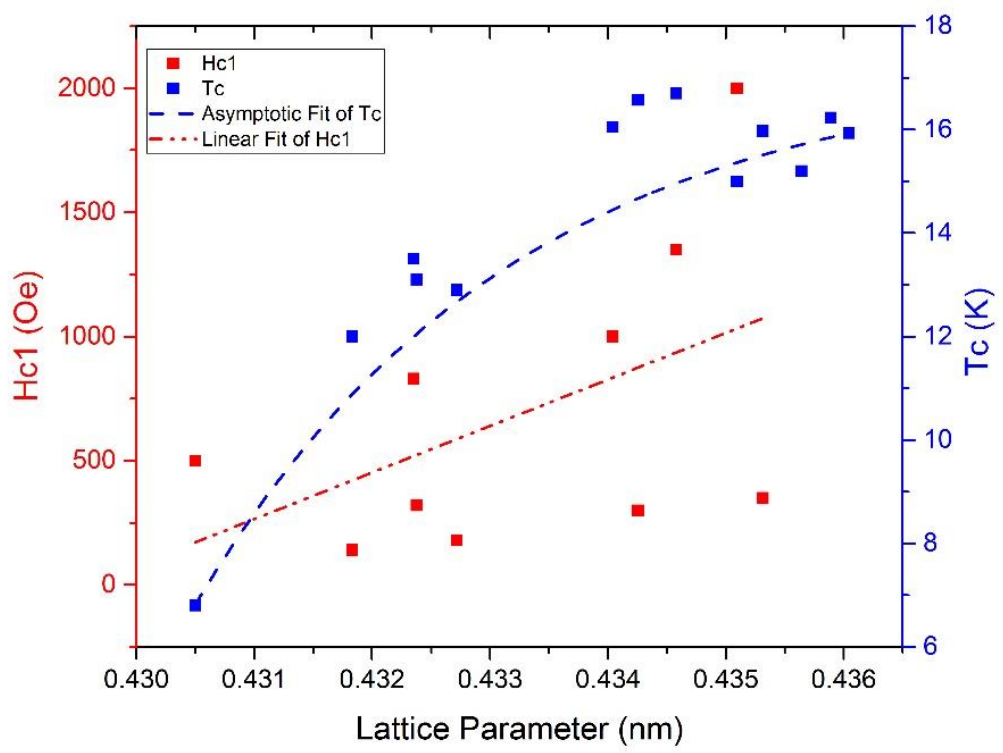

Figure 5.6: $\mathrm{H}_{\mathrm{c} 1}$ vs Lattice Parameter vs $\mathrm{T}_{\mathrm{c}}$ Graph of NbTiN samples studied. Tc data exhibits an asymptotic approach to a "bulk" value while $\mathrm{H}_{\mathrm{c} 1}$ data shows a general trend towards some "bulk" value which is expected due to the dependence of $\mathrm{H}_{\mathrm{c} 1}$ on other variables. Large variability in sample performance showcases need for precise process control, especially when depositing films in the unstable reactive sputtering regime. 
One important result from the work presented is the observed relationship between lattice parameter and the superconducting properties $\left(H_{c 1}\right.$ and $\left.T_{c}\right)$. It is well known that superconducting properties are highly dependent on the composition and microstructure of the sample; since we have established that all films grew epitaxially, the dependence of the measured superconducting values with lattice parameters reported in Figure 5.6 is a clear indication of the relationship between superconducting properties and film composition or stoichiometry. We observe that $\mathrm{H}_{\mathrm{c} 1}$ and $\mathrm{T}_{\mathrm{c}}$ exhibit clear increases for increasing lattice parameter approaching bulk values, in contrast with previous reports [55].

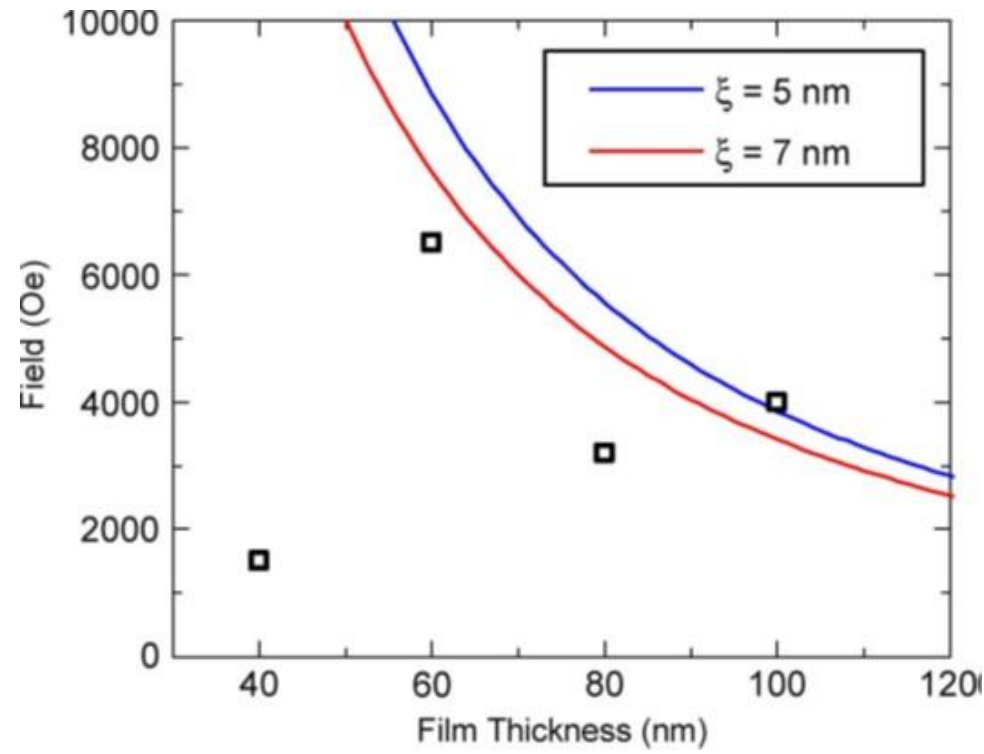

Figure 5.7: Graph of the $\mathrm{H}_{11}$ enhancement of $\mathrm{MgB}_{2}$ films measured with the theoretical enhancement plot shown. [44]

One important note to make with respect to the $\mathrm{H}_{\mathrm{c} 1}$ enhancements shown above for $\mathrm{NbN}$ and $\mathrm{NbTiN}$ films is their underperformance as compared to the theoretical predication. Every sample measured in this research showed significantly lower $\mathrm{H}_{\mathrm{c} 1}$ 
enhancements than predicted in theory. These results showcase the discrepancy between the bulk-like structure of the films assumed in theory, although no true bulk values exist for NbTiN, and the structure obtained using the deposition methods employed above. However, results from $\mathrm{MgB}_{2}$ films [44], displayed in Figure 5.7, show measured $H_{c 1}$ enhancements on the same order as the proposed theoretical enhancements which gives more evidence to the less than bulk-like structure of the $\mathrm{NbN}$ and NbTiN films created since the $\mathrm{MgB}_{2}$ films were deposited using a hybrid CVD-PVD technique that has been shown to produce higher quality films with intrinsically more bulk-like structure than films created using pure PVD techniques, as those used for this research. In conclusion, our correlated structure-property studies reveal the strong link between composition, microstructure and physical properties of thin films, in particular for the case of compound thin films shown here, which are inherently more difficult to tailor, and for which a more complete consideration of the energetics of each deposition technique have to be taken into account as well. This area is still not fully modeled by current theoretical approaches. 


\section{CHAPTER 6}

\section{Nb Thin Film SRF Cavities}

$\mathrm{Nb} / \mathrm{Cu}$ thin film cavities have been made for and are still used in many SRF accelerators [5] [6] [7] [8] [56]. While previous attempts were met with limited success due to the strong Q-slope present in all cavities reported, the desire for pushing thin film SRF cavity performance, thereby expanding the range of utility, has not diminished and, with the advent of new energetic condensation techniques, the possibility of creating new thin film cavities with improved microstructure, surface morphology and adhesion compared to early attempts is now possible. The work discussed in this section is the result of work done at Jefferson Lab and presented at conferences and published in their respective proceedings [57].

\subsection{Experimental Setup}

For this work, HiPIMS was employed due to its ability to create metal-ion rich plasmas and its easy transferability to existing DCMS cavity deposition designs, thus allowing quick adoption and results. Small coupon samples and cavities were coated using the JLab cavity deposition system, shown in Figure 4.2. This system contains features designed to allow deposition of small samples via a load-lock port and an isolatable cavity attachment section in order to prevent venting the entire vacuum system for each cavity mounting, with the consequent loss of time and potential for increased contamination. A 
small sample study of $\mathrm{Nb}$ films on $\mathrm{Cu}$ substrates was performed in order to investigate the effect of peak pulse power on the resulting $\mathrm{Nb}$ film properties. Cu substrates were obtained from a commercial supplier, MTI Corporation, and come pre-cut and polished to an average reported roughness of approximately $5 \AA$. Prior to growth, the substrates were ultrasonically cleaned in an alternating acetone and methanol bath for 10 minutes each.

Two cavity designs were prepared in-house and utilized for deposition, namely multiple 1.3 GHz low surface field $\mathrm{Cu}$ cavities and a $1.5 \mathrm{GHz}$ C100 end cell low loss $\mathrm{Nb}$ cavity. Every cavity went through many pre-processing steps prior to thin film deposition and after RF testing. For the Nb cavity, prior to the initial baseline RF test, the cavity was exposed to a buffered chemical polish (BCP) etch followed by a high-pressure rinse (HPR). Before coating, the cavity was degreased, cleaned and assembled and sealed in a cleanroom to limit particulate contamination on the deposition surface. After the cavity was coated with a fresh $\mathrm{Nb}$ film and RF tested, the cavity had the film stripped from the interior surface by an adequately long BCP etch based upon the thickness of the deposited $\mathrm{Nb}$ film. For the $\mathrm{Cu}$ cavities, the surfaces were initially mechanically polished using centrifugal barrel polishing (CBP) for several rounds of decreasing polishing media size to obtain mirror-like surfaces. Afterward, the cavities underwent a quick chemical polishing on the interior surface. This step is crucial for creating a good substrate surface for film deposition since there is polishing media buried within the surface from the CBP process that must be removed to expose the clean, smooth crystal grains underneath. This step is followed immediately by a $20 \%$ sulfamic acid rinse used to remove the native oxide of 
the $\mathrm{Cu}$ material and replace it with another, although the oxides are removed during subsequent baking of the Cu cavities at $350^{\circ} \mathrm{C}$ under vacuum prior to deposition.

As a note, Cu electropolishing was initially employed for chemical polishing of $\mathrm{Cu}$ cavities before deposition, however, upon further investigation it was discovered that there were difficult geometry challenges to overcome. Namely, the relative difference in distance of the polishing cathode to the narrow beam pipe sections of the cavity and the equator region and the limited conductivity of the electrolyte is such that the current density was much higher on the beam pipe area and no noticeable polishing effects were observed at the equator, where the polishing is most desired. This result was obtained through comparisons of internal optical inspections of cavities performed at JLab before and after polishing steps. To move past this challenge, in the short term, it was decided to employ a rough $\mathrm{Cu}$ etch, which, while creating a less than ideal surface, allowed for the removal of polishing media and a reset of the substrate surface to a consistent state for all resulting depositions. Currently, a process utilizing a chemical etch known as SUBU, which has been used for Cu cavity preparation at other labs, is being used at JLab and efforts are ongoing to overcome the engineering challenges for electropolishing since it is still the ideal method.

After chemical polishing, the cavities are subsequently cleaned using DI water until at least 7 MOhm resistivity is reached for the stagnant water bath and then dried quickly by rinsing the interior surface with clean acetone before transferring to the JLab cleanroom for deposition assembly. For all the assembly stages, coating or RF testing, the cavities were assembled in a ISO-4 cleanroom following stringent contamination control 
procedures. The cavity was also mounted under a portable clean hood that creates a local ISO-5 quality environment in order to keep particulate contamination within the deposition system and on the substrate surface minimal.

After film growth, the structural properties of the coupon samples (lattice parameter, grain size...etc.) were determined via XRD, while the surface morphology was investigated by Olga Trofimova at the Applied Research Center (ARC) at JLab via AFM, using a Digital Instruments Scanning Probe Microscope (SPM) with a tip radius of $<10 \mathrm{~nm}$, and EBSD using the JLab SEM machine in collaboration with Josh Spradlin. The cavity coatings were RF tested at JLab by Dr. Ari Palczewski and Dr. Pashupati Dhakal in the Vertical Test Area (VTA).

\subsection{Coupon Samples}

Multiple series of small samples were deposited using a cylindrical $\mathrm{Nb}$ cathode in a $\mathrm{Kr}$ atmosphere with a fixed target-to-substrate distance of $10 \mathrm{~cm}$, the same approximate distance to the equator region of a $1.3 \mathrm{GHz}$ low surface field SRF cavity and all sample thicknesses were around $300 \mathrm{~nm}$. Specifically, two sample series were performed to observe the effects of changing the peak pulse power and bias between samples. Samples in the power series, shown in Table 6.1, were deposited for two hours each at constant $\mathrm{Kr}$ pressure of $3.9 \mathrm{mTorr}$, substrate bias of $-100 \mathrm{~V}$, substrate temperature of $350^{\circ} \mathrm{C}$, and pulse parameters (frequency of $80 \mathrm{~Hz}$ and pulse width of $100 \mu \mathrm{s}$ ) resulting in thicknesses around $300 \mathrm{~nm}$. The power was then varied between samples ranging from 70 to $400 \mathrm{~kW}$. 
Table 6.1: Table of peak power in HiPIMS pulse and relevant physical properties measured of samples in $\mathrm{Nb} / \mathrm{Cu}$ power series

\begin{tabular}{|l|c|c|c|c|}
\hline Sample & Peak Power $(\mathrm{kW})$ & Lattice Parameter $(\mathrm{nm})$ & Grain Size $(\mathrm{nm})$ & RMS Roughness $(\mathrm{nm})$ \\
\hline A & 69 & 0.33294 & 25.86 & 16.71 \\
\hline B & 143 & 0.33182 & 25.39 & 15.25 \\
\hline C & 211 & 0.33124 & 36.54 & 15.87 \\
\hline D & 282 & 0.33147 & 28.52 & 17.65 \\
\hline E & 350 & 0.33162 & 24.19 & 18.44 \\
\hline F & 391 & 0.33142 & 23.17 & 26.62 \\
\hline
\end{tabular}

From the XRD scan in Figure 6.1, we note that our samples exhibit polycrystalline texturing dominated by the $\mathrm{Nb}(011)$ phase suggesting that heteroepitaxial growth was achieved; this is reinforced by the EBSD results from the $\mathrm{Cu}$ and $\mathrm{Nb} / \mathrm{Cu}$ samples shown in Figure 6.2. Average out-of-plane grain sizes were obtained using the Scherrer equation and the FWHM of the $\mathrm{Nb}(011)$ peak, evidencing that all the samples had grain-size values between 23 and $37 \mathrm{~nm}$. Also, the lattice parameters varied between 0.3312 and 0.3329 $\mathrm{nm}$, which are all slightly greater than the bulk value of $0.33 \mathrm{~nm}$.

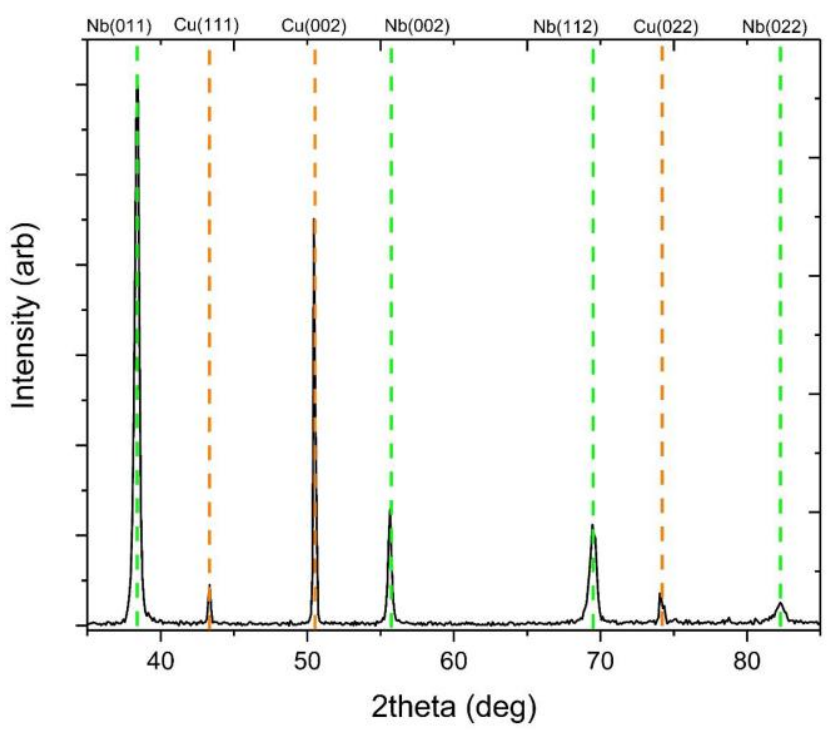

Figure 6.1: Representative XRD scan of $\mathrm{Nb} / \mathrm{Cu}$ samples in power series 
(a)

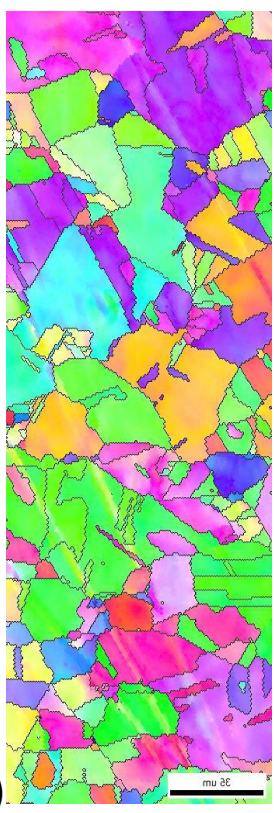

(b)

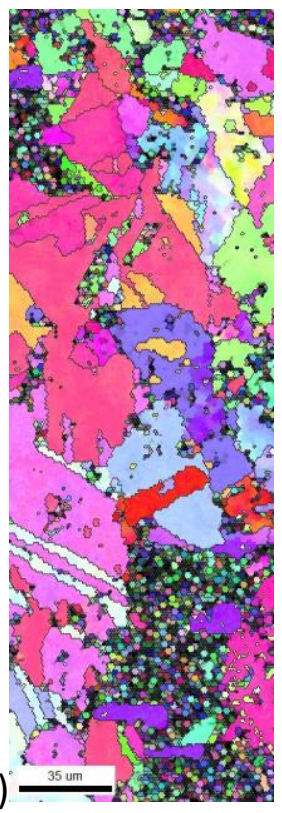

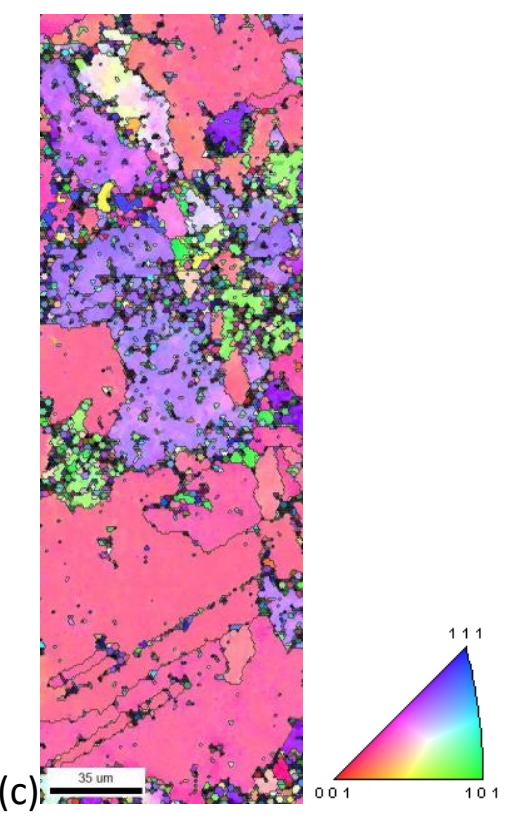

Figure 6.2: EBSD scans performed on $\mathrm{Cu}$ and $\mathrm{Nb} / \mathrm{Cu}$ samples. In order from left to right: (a) an uncoated $\mathrm{Cu}$ substrate, (b) low peak power $\mathrm{Nb} / \mathrm{Cu}$ sample, and (c) high peak power $\mathrm{Nb} / \mathrm{Cu}$ sample. OIM color map included on right for reference of crystal phases.

Analysis of the surface morphology of the samples was performed at the ARC by

Olga Trofimova using the surface profiler system mentioned above. A representative scan of the AFM results can be seen in Figure 6.3. All the samples exhibited RMS roughness values between 15 and $27 \mathrm{~nm}$, on a $50 \times 50 \mu \mathrm{m}$ scale, showing a qualitatively smooth surface similar to the underlying copper substrate. It is noticeable that no sharp features were observed on the surface, which would be detrimental to the RF performance in an actual SRF cavity, however the samples did contain slight pitting, again similar to the $\mathrm{Cu}$ substrate. 


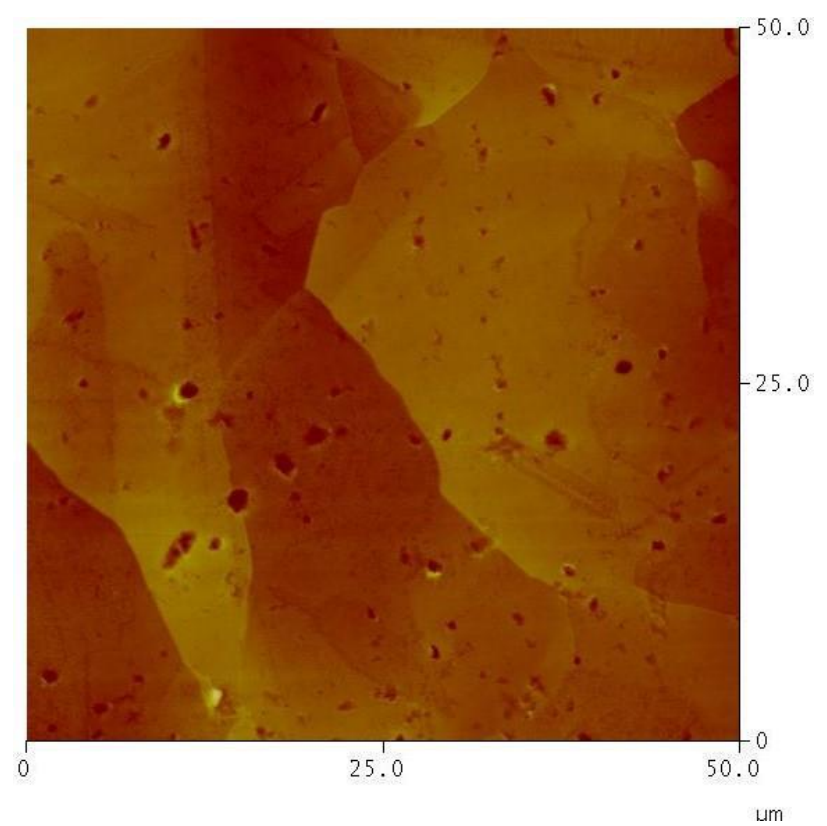

Figure 6.3: Representative AFM scan from $\mathrm{Nb} / \mathrm{Cu}$ sample in power series on a $50 \times 50$ micron scale.

The goal of the small sample power series performed above was to observe correlations between film properties and the applied peak pulse power and to determine the optimal power parameter for performing cavity depositions. When determining the optimal deposition parameters for a film on a cavity, a logical starting point would be to use such parameters that led to films with the most bulk-like properties, such as lattice parameter. Figure 6.4 shows the results from the small sample tests plotting the lattice parameters of the samples and Grain Size versus the peak power and Figure 6.5 shows a plot of the RMS roughness of power series samples versus peak power. As can be seen from the graphs, the parameter set that exhibited the most bulk-like lattice parameter and a minimum in the RMS surface roughness was around $225 \mathrm{~kW}$ peak power. Also, we see the roughness of the samples remains relatively small for lower peak powers but then 
has a strong relationship with increasing power. This information was used to set and hold constant the peak power used for the subsequent bias series.

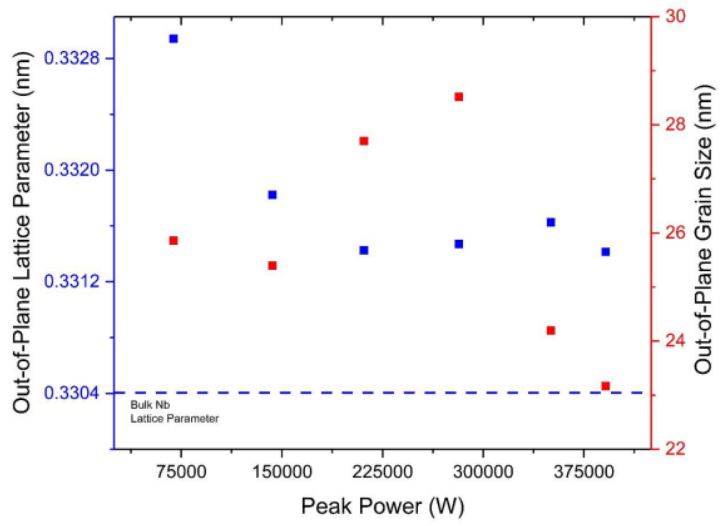

Figure 6.4: Plot of lattice parameter and grain size vs. peak power in $\mathrm{Nb} / \mathrm{Cu}$ power series

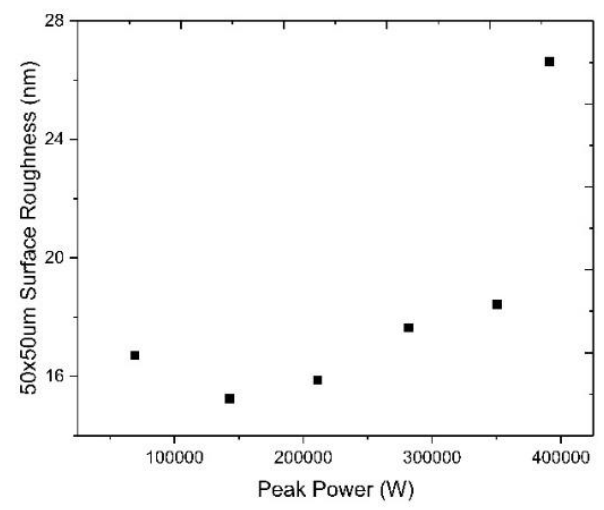

Figure 6.5: Plot of RMS roughness, on 50x50um scale, vs peak power for $\mathrm{Nb} / \mathrm{Cu}$ power series samples

Next, a bias series was performed by varying the bias over the range of 0 to -300 $V$ while attempting to hold constant the peak power, at the above determined setting of $250 \mathrm{~kW}$, obtained from the above power series, the $\mathrm{Kr}$ pressure at $4.2 \mathrm{mTorr}$ and still maintaining the same substrate temperature and pulse parameters as used in the power 
series. The pressure was changed between sample series as a result of having to change the cold-cathode pressure gauge before the bias series was performed. However, since the HiPIMS current-voltage (I-V) curve is extremely sensitive to pressure, a comparative calibration study was done to determine the new pressure that would achieve an equivalent I-V curve to that observed during the power series. Table 6.2 lists the deposition parameters and physical properties measured of the samples contained within the bias series.

Table 6.2: Deposition bias and relevant properties for samples in the $\mathrm{Nb} / \mathrm{Cu}$ bias series

\begin{tabular}{|l|l|l|l|l|}
\hline Sample & Substrate Bias $(-\mathrm{V})$ & Lattice Parameter $(\mathrm{nm})$ & Grain Size $(\mathrm{nm})$ & RMS Roughness $(\mathrm{nm})$ \\
\hline A & 0 & 0.3302 & 20.42 & 16.07 \\
\hline B & 50 & 0.332 & 26.71 & 13.88 \\
\hline C & 100 & 0.3312 & 36.54 & 15.87 \\
\hline D & 200 & 0.3318 & 23.05 & 35.46 \\
\hline E & 300 & 0.3314 & 13.91 & 201.64 \\
\hline
\end{tabular}

The goal of the bias series was the same as that of the peak power series but with interest in the film property dependence on changes in the applied bias. Since HiPIMS pulses contain a much larger fraction of sputtered metal ions than in DCMS, especially of the sputtered metal species, the applied bias will increase the deposition energy of the system and, as discussed previously, should result in increased film quality compared to DCMS methods, as suggested by the SZD diagram presented in Chapter 3. In Figure 6.6 we can see the dependence of the lattice parameter and grain size on substrate bias, while Figure 6.7 shows the RMS roughness of the samples versus applied substrate bias. There are some important features to extract from the information plotted in this figure. First, the RMS roughness of the samples, while remaining low at lower bias values, 
increases dramatically with increasing bias. This gives a practical boundary to the applied bias for a specific peak power setting due to the unreasonably large surface roughness created. Second, the lattice parameter at low bias exhibits a lower than bulk value while, as the bias is increased, the lattice parameter increases quickly to larger than bulk before and eventually plateauing. This is perhaps the most important information to draw from the data in the bias series because it exhibits the ability to tailor properties of the materials being used.

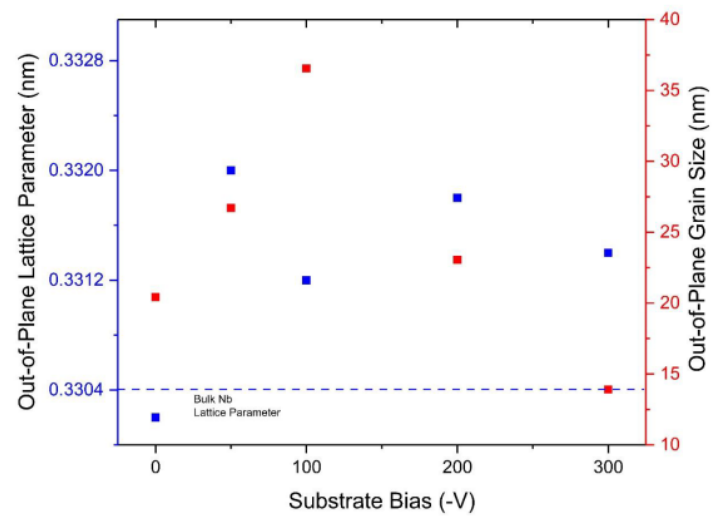

Figure 6.6: Plot of lattice parameter and grain size vs. applied bias in $\mathrm{Nb} / \mathrm{Cu}$ bias series

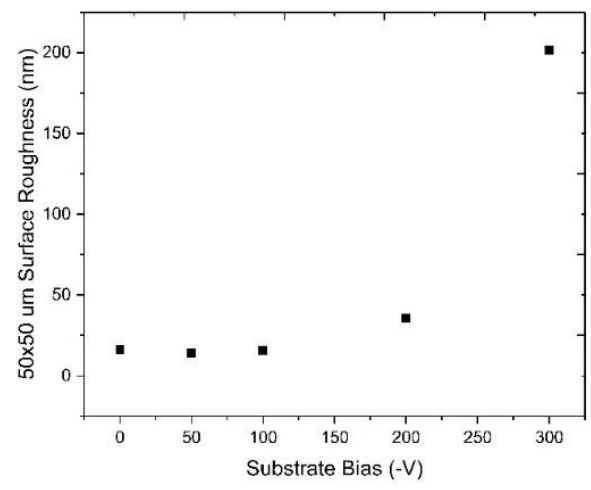

Figure 6.7: Plot of RMS roughness, on 50x50um scale, vs applied bias for $\mathrm{Nb} / \mathrm{Cu}$ bias series samples. Note the roughness scale difference compared to the power series results above. 
Based on these results, it is observed that surface engineering could have beneficial applications to SRF cavities. Somewhat counterintuitively, this shows that even though the field is increasingly moving to attempt to overcome thin film issues with highenergy deposition, the film with the most bulk-like microstructure is found in the film with the lowest bias, or energy. In fact, it has been reported [58] that the highest performing HiPIMS cavity made to date, in terms of $Q$ value at low field, was done with no substrate bias applied during deposition. Using this fact, it may be useful to attempt a deposition process in which the early stages of growth and nucleation are dominated by high-energy deposition with a large substrate bias in order to improve adhesion between film and substrate, followed by a manipulation of the bias voltage to favor better film structure and RF performance in the crucial active SRF surface thickness of the film.

\subsection{Cavity RF Results}

Using a baseline set of deposition parameters, cavities were coated with an approximately one-micron thick $\mathrm{Nb}$ film to test the resulting RF properties. The first cavity coatings shown here were deposited on a $1.5 \mathrm{GHz}$ Nb SRF cavity. In the early stages of the project, Cu cavities were attempted without success. Upon investigation, and four coating attempts, it was discovered that the electron beam welds performed during the

manufacturing process were not suitable for thin film deposition or good cavity performance due to the fact that, for nearly every cavity made, the weld parameters were insufficient for $\mathrm{Cu}$ material and yielded welds that were incomplete and left large cracks 
at the weld areas. These features, while detrimental by themselves, were exacerbated by the subsequent chemistry, performed where the chemicals used were trapped in cracks and left to seep out and pollute the Cu surface prior to deposition, ultimately interfering with the good growth desired of the $\mathrm{Nb}$ films, as shown in Figure 6.8. While work was being done to fix this issue for future cavities, and some current ones, it was desired to test the capabilities of the JLab cavity deposition system. Therefore, an approach was adopted in which a $\mathrm{Nb}$ cavity could be used as the substrate surface which, while not exactly comparable to $\mathrm{Cu}$, would give a reliable surface and a rough indication of the cavity coating capabilities.

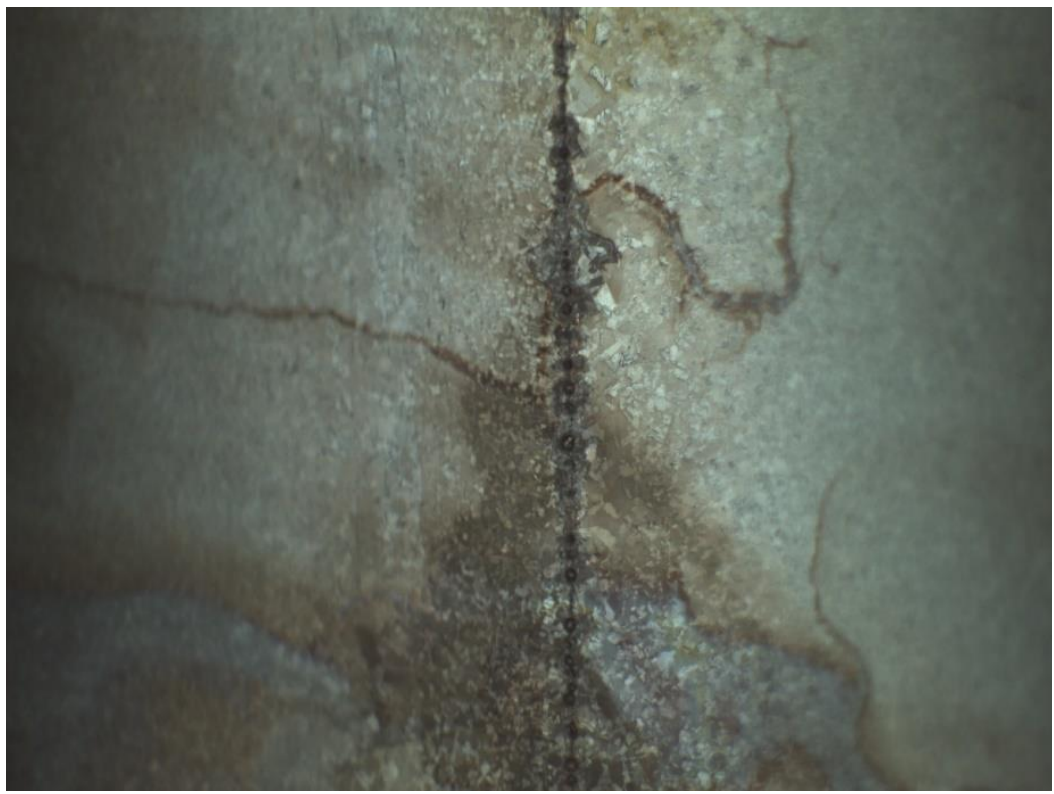

Figure 6.8: Internal inspection photo of Cu cavity after EP showcasing equator weld crack and chemical trapping.

The first cavity coating was deposited using a preliminary design for the cavity deposition system immediately before the entire system was overhauled, cleaned and rebuilt with a multitude of upgrades to the vacuum hardware while keeping the overall 
deposition design fundamentally unchanged. Therefore, the second cavity coating was meant as a confirmation of the consistency between the RF results of the two films and system designs.
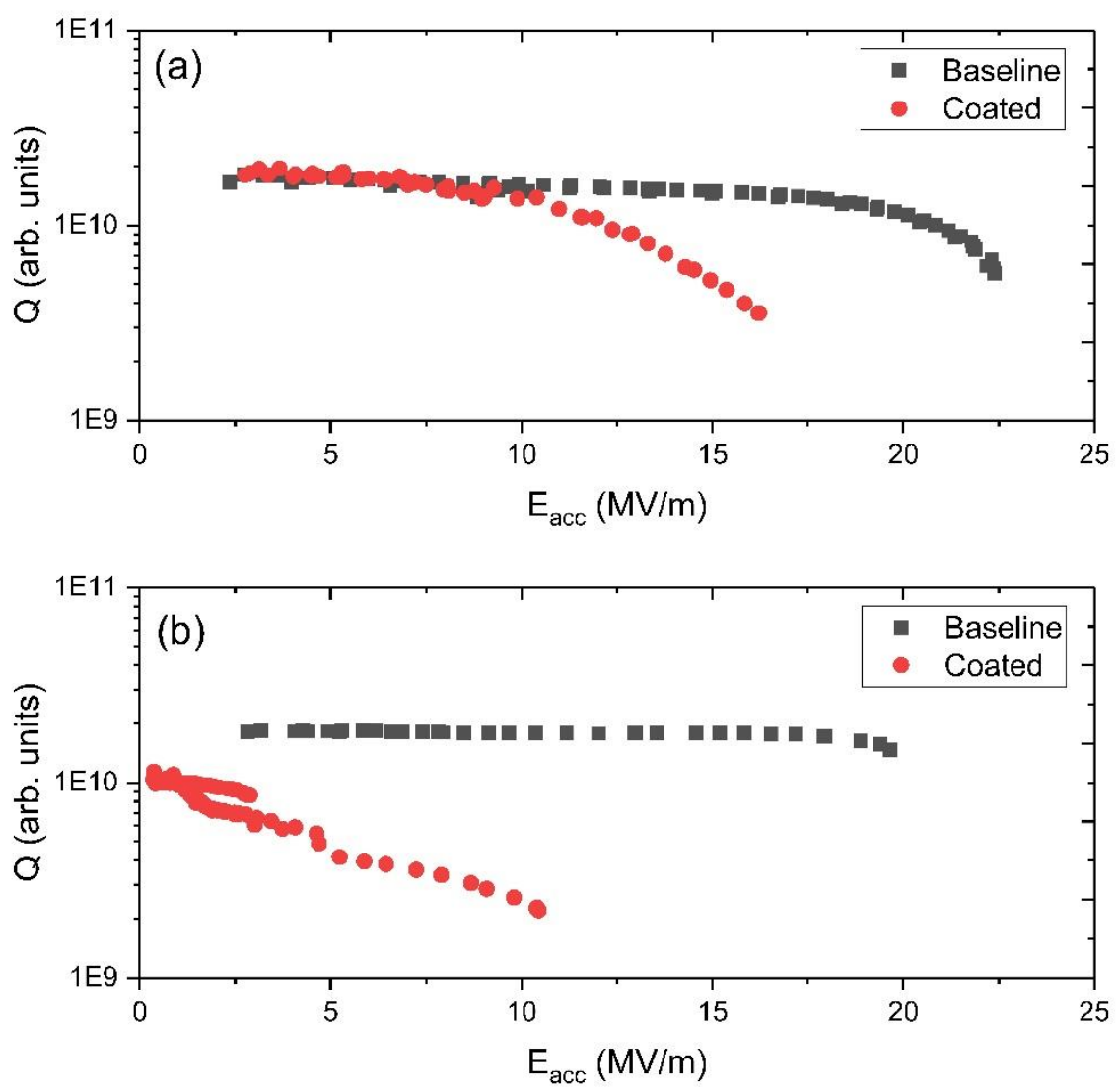

Figure 6.9: Plot of the Quality Factor of the Nb cavity before (black squares) and after (red circles) coating. (a) corresponds to the first coating attempt done at JLab while (b) corresponds to the second. Coating (a) exhibits high low-field $Q$ and shallow Q-slope up to $10 \mathrm{MV} / \mathrm{m}$ while coating (b) exhibits behavior indicative of a thermal switch.

The RF tests were performed at JLab in the VTA where a large variety of accelerating structures are tested on a regular basis. The main graph of interest in the present study is the cavity Q-value vs the accelerating gradient. Since the main challenge facing thin film cavities is the $Q$-slope, it is valuable to observe the relative $Q$-slope between various thin film cavities and compare how the low-field $Q$ values behave for 
various parameters. Figure 6.9 shows the $\mathrm{RF}$ results for both $\mathrm{Nb} / \mathrm{Nb}$ cavity coatings done at JLab overlaid with their respective baseline RF test results. As can be seen in Figure 6.9(a), the first cavity coating showed a low-field $\mathrm{Q}$ equivalent to that of the bulk $\mathrm{Nb}$, perhaps even slightly larger than baseline, and maintained a reasonably flat $Q$ up to 10 $\mathrm{MV} / \mathrm{m}$. The second cavity coating yielded more unique results. The graph, in Figure 6.9(b), shows a good low-field $Q$, but also a sharp drop in the $Q$ value around $1.5 \mathrm{MV} / \mathrm{m}$. This drop is also part of a hysteresis curve. The behavior exhibited here is indicative of a socalled "thermal switch." This is a phenomenon usually occurring due to a nonsuperconducting contaminant on the surface of the RF cavity. As the applied power is increased, the contaminant begins to heat up and raise the temperature of the surrounding cavity, thus causing a small lowering of the temperature dependent $Q$ value. At a certain point, this causes a small area to rise above the critical temperature and results in a sharp decrease in the cavity Q. Due to the transient nature of superconductors this scenario then has a different behavior when reducing the applied field and cooling resulting in a hysteresis curve as observed.

After the cavity system was shown to be able to deposit $\mathrm{Nb}$ films at least at a level comparable to previous and current attempts, the task of coating Cu cavities was revisited. While the cavity deposition system was undergoing upgrades and a rebuild after the $\mathrm{Nb}$ cavity depositions, the weld issues discussed previously were solved and five new $1.3 \mathrm{GHz}$ $\mathrm{Cu}$ cavities were created and some existing cavities were repaired. These, now good, cavities were then coated in order to obtain a baseline value for performance. However, as you can see from Figure 6.10, the RF results were consistently well below the 
anticipated level of around bulk $\mathrm{Nb}$ for low accelerating gradient. Here the project encountered a second engineering challenge. Upon inspection, the cavities were found to be incompletely covered by the $\mathrm{Nb}$ film with a large section of the far beam pipe left completely uncovered. The heat generated by the $\mathrm{Cu}$, even in the low fields of the beam pipes, helps explain the low RF performance. Investigations into this problem discovered that the cavity deposition system, upon rebuild, incorporated a flaw in the design of the magnetron support tubes. Specifically, the anode support tube had been upgraded to a more robust design that, unfortunately, was too large in diameter to fit completely within the cavity. This then limited the distance the $\mathrm{Nb}$ cathode could be inserted into the cavity and only allows about $80-90 \%$ of the cavity to be coated in one run. Work is now ongoing to fix this problem by increasing the $\mathrm{Nb}$ cathode and anode grid length to accommodate this new feature, since the $\mathrm{Nb}$ target cathode is depleted and needs to be replaced.
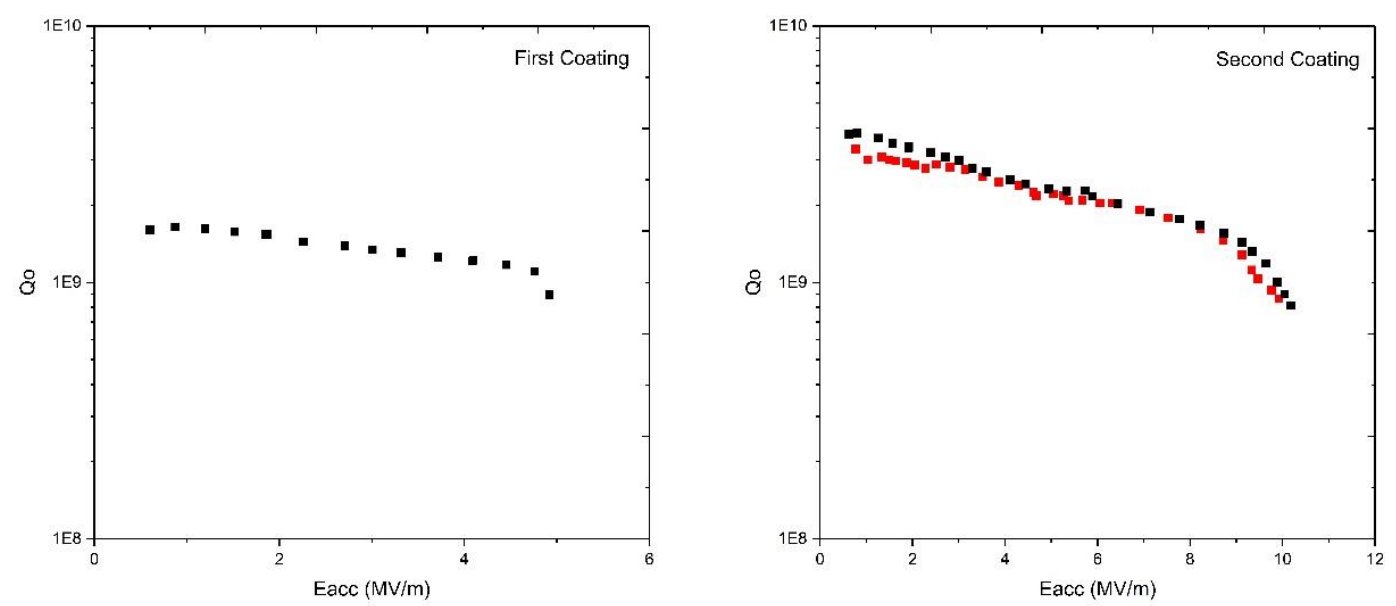

Figure 6.10: Graphs of the $Q$ vs $E$ curves measured for two Cu cavity coating attempts. Cavities exhibited lower $Q$ values than expected and were residual resistance dominated. This was later found to be a result of incomplete coatings. 
The results presented here have shown that HiPIMS has great promise for future work in creating high-quality superconducting thin films for RF applications. We have created small coupon samples with bulk-like lattice parameters and relatively smooth surfaces free from sharp defects that are detrimental to RF surfaces. The preliminary results on cavities have also shown promise. We have achieved a Nb thin film cavity with a $Q$ value equal to that of its bulk Nb counterpart at low field and remaining relatively flat up to $10 \mathrm{MV} / \mathrm{m}$. Even with the observed thermal switch present in the second cavity coating, the results are promising for future SRF thin film cavities; they give a starting point to begin pursuing control over the flaws observed in the technology over the last 40 years. Overall, the results of films deposited on small coupon samples and cavities have shown good results and show promise for HiPIMS technology to be used to create high quality SRF thin films. 


\section{CHAPTER 7}

\section{Conclusion}

Throughout this thesis, the enabling capabilities of thin films has been discussed and shown. The results of the films presented herein showcase the high reproducibility of thin films as well as the vast matrix of parameter combinations and resulting properties possible using this technology for specific applications. Superconducting $\mathrm{Nb}$ thin films have been presented with microstructure that was precisely controlled and achieved near bulk-like. $\mathrm{NbN}$ and $\mathrm{NbTiN}$ thin films with thicknesses lower than their penetration depth have been created that exhibit the theoretically predicted increase in the lower critical field enhancement and showcase the bright future of this phenomenon's application to SRF accelerating cavities. A major advancement in this thesis is the example of the construction and adaptation of new energetic condensation techniques to the unique and difficult cavity geometries giving the groundwork and vision for future work to be done on the application of thin films and these new deposition techniques to deployable cavities.

However, applying thin films to SRF accelerator cavities needs much more work in the near future to overcome many challenges in order to fully realize their potential. The biggest challenge to overcome is the observed $\mathrm{Q}$-slope defect in current $\mathrm{Nb}$ thin film cavities. The main proposed causes of Q-slope are: poor adhesion leading to micro- 
delamination and thermal feedback, poor film microstructure and magnetic effects. Briefly, we will discuss some concepts of future work to be done and experiments that may be performed to help elucidate the issue. First, the poor adhesion of cavities has been proposed as a Q-slope cause by Dr. Enzo Palmieri and his group [9]. In this theory, the proposed mechanism for Q-slope is based upon the assumption of poor adhesion of the $\mathrm{Nb}$ film leading to micro-delamination of the film from the substrate, backed by experimental observations of peeling in CERN Nb/Cu cavities. In turn, as the applied field is increased in the cavity and heat is generated, these delamination points act as heat flow blocks and lead to a thermal feedback loop which results in a lowering of the cavity Q value as the observed Q-slope.

Some proposed solutions to this problem include: ion stitching to create a very well adhered film and grated interface, stress reduction of the film, very thick films to reduce the solid angle and effect of the delamination points, and improved surface preparation such as seamless cavities and well-applied EP. In order to test these ideas, one of the most straightforward and effective experiments may be to perform ion stitching or high-energy ion bombardment during the early stages of film growth. This would lead to a very well-adhered film across the entire surface and give insight into the effect of this mechanism. The new energetic condensation techniques being adopted by the SRF thin film field, such as HiPIMS and ECR, are directly adaptable to this method since they result in heavily metal ion-rich plasmas ideal for this application. While the process would require an extremely large bias voltage, it is not an insurmountable engineering 
hurdle to overcome and the bias could be lowered throughout the deposition to create a film with good microstructure at the SRF surface, as discussed in Chapter 6.

The second proposed cause of the Q-slope is the poor microstructure of the films when compared to their bulk counterparts. Film microstructure is heavily dependent on film deposition technique $\&$ conditions and substrate quality. The substrate is the initial template for film growth and poor quality of the substrate can lead to increased stress, defects and contamination of the film. Therefore, some of these issues are strongly coupled and great care should always be taken to create an as perfect as possible substrate surface. Solutions to this problem are already underway in many laboratories. Beyond substrate quality, the new energetic condensation techniques have been shown to be capable of depositing films of vastly improved microstructure and surface morphology as compared to the lower-energy methods employed in the past. To fully discern the magnitude of this effect, detailed studies correlating film structure to RF performance, both in cavities and small coupon samples, should be performed and the effects observed.

Also, a novel technique may be employed in order to reduce gas burial, and its negative effect on film quality, during deposition. It has been shown that due to the mass difference between the $\mathrm{Nb}$ ions created in a HiPIMS plasma and the working gas ions, that each species, and ionization, arrive at the substrate separately. This unique condition implies the possibility of employing a variable substrate bias that is either positive or neutral when gas ions impinge first, to either reject them or not give them energy, and then subsequently a negative bias is applied to give energy to the desired metal ions as 
they approach the surface. This technique has yet to be utilized for film deposition, but could have a significant impact on film performance, especially for applications as sensitive as SRF.

Magnetic effects are the last proposed cause of the observed Q-slope. Originally, $\mathrm{Nb}$ thin film cavities were favored due to their apparent lack of sensitivity to the ambient magnetic field during cooldown and operation [5]. This helped accelerators in a practical sense by reducing the amount of shielding needed and therefore the ultimate cost. However, since thin films are inherently more porous and contain more grain boundaries and defects, the lack of sensitivity may in fact be an observed saturation of the magnetic flux at a large number of pinning sites within the film, which at low field shows as an insensitivity to the ambient field during cooldown. However, these pinning sites may comprise a distribution of much lower energy pinning sites, compared to bulk, that start to de-pin at low field and increases with field to result in the observed Q-slope. In line with this, a source of possible magnetic fields that ignores shielding, is the thermocurrents created from the Kapitza resistance arising from the interface of dissimilar metals, namely $\mathrm{Nb}$ and $\mathrm{Cu}$. If the materials are not held in perfect thermal equilibrium with each other, currents will begin to flow and magnetic fields are created and possibly trapped. This is also detrimental to the possible reduction of the first magnetic effect proposed. Specifically, new techniques in SRF employed for nitrogen-doped cavities are attempting to increase the thermal gradient during cooldown to such an extent as to expel magnetic flux from being trapped in the surface by a thermodynamic force [59]. However, in this application, that would necessarily create more magnetic fields and result in lower 
performance. Therefore, one proposed solution to the magnetic effects is to perform two experiments.

First, an insulating interlayer may be deposited between the Cu substrate and the $\mathrm{Nb}$ film. This insulating layer would eliminate the thermocurrents from being created and offer the ability to test thin film cavities without the fields being created and perform RF tests with varied cooldown and flux expulsion rates. Second, it is possible for a cavity to be coated and RF tested without ever exposing the $\mathrm{Nb}$ film surface to air, or anything but vacuum. The JLab cavity deposition system was specifically designed with this test in mind by offering the ability to deposit a cavity with movable RF measurement probes already installed and then transfer the system under UHV, by closing the accompanying gate valve, to an appropriate testing stand where the vacuum is then re-established before the gate valve is opened again. This test would not allow the film to oxidize and create flux trapping sites, which may especially be important for the numerous grain boundaries in the film. As a result, the effect of Josephson vortices on the RF performance could be observed for the first time.

Beyond $\mathrm{Nb}$ films, the new materials, some of which were researched here, are very promising to future SRF technology advances. The materials offer the ability to create accelerating structures with higher maximal accelerating gradients and operating temperatures than bulk $\mathrm{Nb}$. Materials such as $\mathrm{Nb}_{3} \mathrm{Sn}$, which is already being tested for cavity application, is a perfect example of the possible advances from the implementation of new materials [60]. Higher accelerating gradients would greatly increase the applications for accelerators by allowing higher energy physics to be explored and for 
smaller, more economical accelerators to be implemented in new areas where size was previously a limiting factor. Also, the increased operating temperature, while maintaining efficiency, and no longer needing to necessarily operate in the superfluid liquid helium regime, would drastically reduce the operating costs of many accelerators, allowing more institutions to have access to them where it wasn't possible before.

However, multilayer films and new materials have many challenges ahead. First, adequate control must be obtained and exhibited over film stoichiometry and microstructure before adequate implementation can be achieved. This was shown dramatically in the results presented in Chapter 5 on NbTiN. The NbTiN films created exhibited variability in properties and structure due to their strong sensitivity to process control. In order to apply these films successfully to cavities more work must be done on creating reproducible films with bulk-like structure.

The research done here showcases the path forward in this by making apparent the need for precise control throughout the deposition process through methods like mass-flow controllers and co-deposition. Fundamental studies continue to be needed, however, much of the work also needs to be transferred to setups that are capable of coating actual SRF cavities. The studies would then be transferrable to cavities and give better insight into the correlations between structure and RF performance. Also, detailed studies need to be done in these systems showing the conformality of film thickness and quality over large, cavity-like surfaces while also observing and improving on the crucial interfaces necessary in the SIS model. Perhaps a first step would be the development of deposition systems capable of coating cavities with multilayers or multiple systems where 
cavities may be transferred under vacuum between them in order to receive the coatings of different materials. Lastly, the ultimate property of importance is the RF performance of coated cavities. More efforts need to be pursued in order to develop and test thin film coated cavities for it is in diversity we will see rapid progress made in this topic.

In closing, thin films offer endless possibilities in material property tailoring and application to existing and future technology. SRF is a field which could benefit dramatically from investment into and development of this technology by allowing the creation of accelerators with reduced cost and unprecedented accelerating gradients. This would allow for accelerators to be a more widely-used technology and reach energies - and physics - never before achieved. The future is indeed bright for thin film technology. Thin films are already a highly-desired technology for future accelerators and with the proper investment the next leaps forward are not far away. It will be amazing to see the progress obtained in the near future and the great benefits that will result. It is my hope that by reading this thesis one may be encouraged and possibly inspired about the future possibilities and benefits thin films offer. 


\section{Bibliography}

[1] M. Ohring, Materials Science of Thin Films: Deposition and Structure, Academic Press, 2002.

[2] H. Luth, Solid Surfaces, Interfaces and Thin Films, New York: Springer, 2015.

[3] D. M. Mattox, Handbook of Physical Vapor Deposition (PVD) Processing, William Andrew, 2010.

[4] P. M. Martin, Handbook of deposition technologies for films and coatings: science, applications and technology, William Andrew, 2009.

[5] S. Calatroni, "20 Years of Experience with the $\mathrm{Nb} / \mathrm{Cu}$ Technology for Superconducting Cavities and Perspectives for Future Developments," in Physics C: Superconductivity, Ithaca, 2005.

[6] C. Benvenuti, S. Calatroni, P. Darriulat, M. A. Peck, A. -M. Valente and C. A. Van't Hof, "Study of the Residual Resistance of Superconducting Niobium Films at $1.5 \mathrm{GHz}$," in Proceedings of the 1999 Workshop on RF Superconductivity, Santa Fe, NM, USA, 1999. 
[7] C. Benvenuti, S. Calatroni, M. Hakovirta, H. Neupert, M. Prada and A. Valente, "CERN Studies on Niobium-Coated 1.5 GHz Copper Cavities," in 10th Workshop on RF Superconductivity, Tsukuba, 2001.

[8] S. Calatroni, E. Barbero-Soto, C. Benvenuit, L. Ferreira and H. Neupert, "Progress of Nb/Cu Technology with 1.5GHz Cavities," in 11th Workshop on RF Superconductivity, Lubeck, 2003.

[9] V. Palmieri and R. Vaglio, "Thermal Contact Resistance at the $\mathrm{Nb}-\mathrm{Cu}$ Interface," in Prceedings of the SRF2015, Whistler, BC, CA, 2015.

[10] B. Visentin, "Review on Q-DROP Mechanisms," in International Workshop on Thin Films and New Ideas for Pushing the Limits of RF Superconductivity, 2006.

[11] A. Gurevich, "Enhancement of RF Breakdown Fild of Superconductors by Multilayer Coating," Applied Physics Letters, vol. 88, p. 012511, 2006.

[12] A. Gurevich, "Maximum screening fields of superconducting multilayer structures," AIP Advances, vol. 5, no. 1, p. 017112, 2015.

[13] A. Gurevich, "Multilayer coating of superconducting cavities: challenges and opportunities," in Proceeding of 2011 SRF Workshop, Chicago, 2011.

[14] T. Kubo, "Theory of multilayer coating for proof-of-concept experiments," in SRF2015, Whistler, 2015. 
[15] M. S. Livingston, Particle Accelerators: A Brief History, Cambridge: Harvard University Press, 1969.

[16] A. Nassiri, B. Chase, P. Craievich, A. Fabris, H. Frischholz, J. Jacob, E. Jensen, M. Jensen, R. Kustom and R. Pasquinelli, "History and Technology Developments of Radio Frequency (RF) Systems for Particle Accelerators," IEEE Transactions on Nuclear Science, vol. 63, no. 2, 2016.

[17] A. Georges and et al, "Observation of a new particle in the search for the Standard Model Higgs boson with the ATLAS detector at the LHC," Physics Letters B, vol. 716, no. 1, pp. 1-29, 2012.

[18] J. N. Galayda, "The LCLS-II Project," in Proceedings of IPAC 2014, Denver, 2014.

[19] A. Schwettman, P. B. Wilson, J. M. Pierce and W. M. Fairbank, International Advances in Cryogenic Engineering, vol. 10, no. 88, 1965.

[20] G. Ciovati, P. Dhakal and G. R. Myneni, "Superconducting radio-frequency cavities made from medium and low purity niobium ingots," Superconductor Science and Technology, vol. 29, no. 6, p. 064002, 2016.

[21] P. Dhakal, G. Ciovati, P. Kneisel and G. R. Myneni, "Analysis of post wet chemistry heat treatment effects on Nb SRF surface resistance," arXiv preprint arXiv:1310.8159. 
[22] P. Dhakal, G. Ciovati, P. Kneisel and G. R. Myneni, "Reproducibility of high-Q SRF cavities by high-temperature heat treatment," in Proceedings of IPAC 2014, Denver, 2014.

[23] P. Dhakal, G. Ciovati, G. R. Myneni, N. Groll, P. Naheshwari, D. M. McRae, R. Pike, T. Proslier, F. Stevie, R. P. Walsh, Q. Yang and J. Zasadzinzki, "Effect of high temperature heat treatments on the quality factor of a large-grain superconducting radio-frequency niobium cavity," Physical Review Special Topics-Accelerators and Beams, vol. 16, no. 4, p. 042001, 2013.

[24] H. Padamsee, J. Knobloch and T. Hays, RF Superconductivity for Accelerators, John Wiley \& Sons, Inc., 1998.

[25] V. Z. Kresin and S. A. Wolf, Fundamentals of Superconductivity, New York: Plenum Press, 1990.

[26] A. S. Alexandrov, Theory of Superconductivity: From Weak to Strong Coupling, London: Institute of Physics Publishing, 2003.

[27] M. L. Cohen and S. G. Louie, Fundamentals of Condensed Matter Physics, Cambridge University Press, 2016.

[28] K. Masek and V. Matolin, "RHEED study of Nb thin film growth on $\mathrm{Cu}$ (111) and (100) single-crystals," Vacuum, vol. 61, no. 2, pp. 217-221, 2001. 
[29] A. Anders, "A structure zone diagram including plasma-based deposition and ion etching," Thin Solid Films, vol. 518, pp. 4087-4090, 2010.

[30] U. Helmersson, M. Lattemann, J. Bohlmark, A. P. Ethiasarian and J. T. Gudmundsson, "Ionized Physical Vapor Deposition (IPVD): A Review of Technology and Applications," Thin Solid Films, vol. 513, no. 1, pp. 1-24, 2006.

[31] A.-M. Valente-Feliciano, S. Aull, G. Eremeev, T. Proslier, C. E. Reece and J. Spradlin, "Material quality \& SRF performance of Nb films grown on Cu via ECR plasma energetic condensation," in 17th International Conference on RF Superconductivity (SRF2015), Whistler, 2015.

[32] J. Bohlmark, Fundamentals of High Power Impulse Magnetron Sputtering, PhD Dissertation No. 1014, Linkoping University Institute of Technology, 2006.

[33] A. Anders, "Physics of Plasma-Based lon Implantation \& Deposition (PBIID) and High Power Impulse Magnetron Sputtering (HiPIMS): A Comparison," physica status solidi (a), vol. 205, no. 4, pp. 965-970, 2008.

[34] A. P. Ehiasarian, J. G. Wen and I. Petrov, "Interface microstructure engineering by high power impulse magnetron sputtering for the 
enhancement of adhesion," Journal of Applied Physics, vol. 101, p. 054301, 2007.

[35] M. Samuelsson, D. Lundin, K. Sarakinos, F. Bjorefors, B. Walivaara, H. Ljungrantz and U. Helmersson, "Influence of ionization degree on film properties when using high power impulse magnetron sputtering," Journal of Vacuum Science and Technology A: Vacuum, Surfaces, and Films, vol. 30, no. 3, p. 031507, 2012.

[36] M. Samuelsson, D. Lundin, J. Jensen, M. A. Raadu, J. T. Gudmundsson and U. Helmersson, "On the Film Density Using High Power Impulse Magnetron Sputtering," Surface and Coatings Technology, vol. 205, no. 2, pp. 591-596, 2010.

[37] K. Sarakinos, J. Alami and S. Konstantinidis, "High power pulsed magnetron sputtering: A review on scientific and engineering state of the art," Surface \& Coatings Technology, vol. 204, pp. 1661-1684, 2010.

[38] K. Shih, X-ray diffraction : Structure, principles, and applications, Hauppauge, New York: Nova Science, 2013.

[39] H. L. Phillips, C. E. Reece, A.-M. Valente-Feliciano, B. Xiao and G. V. Eremeev, "Characterization Of Superconducting Samples With SIC System For Thin 
Film Developments: Status And Recent Results," Thomas Jefferson National Accelerator Facility, Newport News, VA, USA, 2014.

[40] R. Kleindienst, A. Burrill, O. Kugeler and J. Knobloch, "Commissioning results of the HZB quadrupole resonator," No. CERN-ACC-2015-0114, 2015.

[41] M. R. Beebe, D. B. Beringer, M. C. Burton, K. Yang and R. A. Lukaszew, "Stoichiometry and thickness dependence of superconducting properties of niobium nitride thin films," Journal of Vacuum Science \& Technology A: Vacuum, Surfaces, and Films, vol. 34, no. 2, p. 021510, 2016.

[42] D. B. Beringer, PhD in Physics, Williamsburg, VA: The College of William \& Mary, 2017.

[43] M. C. Burton, M. R. Beebe, K. Yang, R. A. Lukaszew, A. Valente-Feliciano and C. E. Reece, "Superconducting NbTiN thin films for superconducting radio frequency accelerator cavity applications," Journal of Vacuum Science \& Technology A: Vacuum, Surfaces, and Films, vol. 34, no. 2, p. 021518, 2016.

[44] D. B. Beringer, C. Clavero, T. Tan, X. X. Xi, W. M. Roach and R. A. Lukaszew, "Thickness Dependence and Enhancement of Hc1 in Epitaxial MgB2 Thin Films," IEEE TRANSACTIONS ON APPLIED SUPERCONDUCTIVITY, vol. 23, no. 3, 2013. 
[45] M. Burton, R. A. Lukaszew, J. Riso, A. Valente-Feliciano, M. Beebe and C. E. Reece, "Superconducting NbN-Based Multilayer and NbTiN Thin Films for the Enhancement of SRF Accelerator Cavities," in 17th International Conference on RF Superconductivity, Whistler, 2015.

[46] W. M. Roach, D. B. Beringer, Z. Li, C. Clavero and R. A. Lukaszew, "Magnetic Shielding Larger Than the Lower Critical Field of Niobium in Multilayers," IEEE Transactions on Applied Superconductivity, vol. 23, no. 3, p. 8600203, 2013.

[47] W. M. Roach, J. R. Skuza, D. B. Beringer, Z. Li, C. Clavero and R. A. Lukaszew, "NbN Thin Films for Superconducting Radio Frequency Cavities," Superconductor Science and Technology, vol. 25, no. 12, p. 125016, 2012.

[48] A.-M. Valente-Feliciano, H. L. Phillips, C. E. Reece and J. K. Spradlin, "SRF Multilayer Structures Based on NbTiN," in Proceedings of SRF 2011, Chicago, 2011.

[49] M. Beebe, PhD in Physics, Williamsburg, VA, USA: The College of William \& Mary, 2017.

[50] D. B. Beringer, "Characterization of Two Different Orientations of Epitaxial Niobium Thin Films Grown on MgO(001) Surfaces," Journal of Applied Physics, vol. 114, no. 22, p. 223502, 2013. 
[51] C. Clavero, D. B. Beringer, W. M. Roach, J. R. Skuza, K. C. Wong, A. D. Batchelor, C. E. Reece and R. A. Lukaszew, "Strain Effects on the Crystal Growth and Superconducting Properties of Epitaxial Niobium Ultrathin Films," Crystal Growth \& Design, vol. 12, no. 5, pp. 2588-2593, 2012.

[52] K. Vasu, M. G. Krishna and K. A. Padmanabhan, "Effect of Nb Concentration on the Structure, Mechanical, Optical and Electrical Properties of NanoCrystalline Ti1-xNbxN Thin Films," Journal of Material Science, vol. 47, p. 3522, 2012.

[53] J. R. Gavaler, D. W. Deis, J. K. Hulm and C. K. Jones, "SUPERCONDUCTING PROPERTIES OF NIOBIUM-TITANIUM-NITRIDE THIN FILMS," Applied Physics Letters, vol. 15, no. 10, pp. 329-331, 1969.

[54] C. Bohmer, G. Brandstatter and H. W. Weber, "The Lower Critical Field of High-Temperature Superconductors," Superconductor Science and Technology, vol. 10, pp. A1-A10, 1997.

[55] H. Myoren, T. Shimizu, T. lizuka and S. Takada, "Properties of NbTiN thin films prepared by reactive DC magnetron sputtering," IEEE transactions on applied superconductivity, vol. 11, no. 1, pp. 3828-3831, 2001. 
[56] G. Terenziani, S. Calatroni, T. Junginger, I. A. Santillana and A. P. Ehiasarian, "Nb Coating Developments with HiPIMS for SRF Applications," in Proceedings of SRF 2013, Paris, 2013.

[57] M. Burton, M. Beebe, R. A. Lukaszew, A. Palczewski, L. Phillips and C. E. Reece, "RF Results of Nb Coated SRF Accelerator Cavities Via HiPIMS," in 18th International Conference on RF Superconductivity, Lanzhou, 2017.

[58] G. J. Rosaz, S. Aull, E. A. Ilyina, A. Sublet and W. V. Delsolaro, "SRF Cavity Coatings: Review of Alternative Materials and Coating Techniques," in SRF 2017, Lanzhou, 2017.

[59] S. Posen, M. Checchin, A. C. Crawford, A. Grassellino, M. Martinello, O. S. Melnychuk, A. Romanenko, D. A. Sergatskov and Y. Trenikhina, "Efficient expulsion of magnetic flux in superconducting radiofrequency cavities for high Q 0 applications," Journal of Applied Physics, vol. 119, no. 21, p. 213903, 2016.

[60] S. Posen and D. L. Hall, "Nb3Sn superconducting radiofrequency cavities: fabrication, results, properties, and prospects," Superconductor Science and Technology, vol. 30, no. 3, p. 033004, 2017. 
[61] L. Zhang, W. Peng, L. X. You and Z. Wang, "Superconducting properties and chemical composition of NbTiN thin films with different thickness," Applied Physics Letters, vol. 107, no. 12, p. 122603, 2015.

[62] N. Holtzer, O. Antonin, T. Minea, S. Marnieros and D. Bouchier, "Improving HiPIMS deposition rates by hybrid RF/HiPIMS co-sputtering, and its relevance for NbSi films," Surface \& Coatings Technology, vol. 250, pp. 3236, 2014.

[63] A. Anders, "A review comparing cathodic arcs and high power impulse magnetron sputtering (HiPIMS)," Surfaces \& Coatings Technology, vol. 254, pp. 308-325, 2014.

[64] C. Z. Antoine, J. C. Villegier and G. Martinet, "Study of the Nanometric Superconducting Multilayers for RF Field Screening Applications," Applied Physics Letters, vol. 102, p. 102603, 2013.

[65] D. M. Glowacka, D. J. Goldie, S. Withington, H. Muhammad, G. Yassin and B. K. Tan, "Development of a NbN Deposition Process for Superconducting Quantum Sensors," IEEE Transactions on Terahertz Science and Technology, vol. 20, no. 20, 2013. 
[66] L. Phillips, K. Macha and A. -M. Valente-Feliciano, "Design and Commissioning Status of New Cylindrical HiPIMS Nb Coating System for SRF Cavities," in Proceedings of SRF2013, Paris, France, 2013.

[67] J. Capek, M. Hala, O. Zabeida, J. E. Klember-Sapieha and L. Martinu, "Deposition rate enhancement in HiPIMS without compromising the ionized fraction of the deposition flux," Journal of Physics D: Applied Physics, vol. 46, p. 205205, 2013.

[68] W. M. Roach, D. B. Beringer, J. R. Skuza, W. A. Oliver, C. Clavero, C. E. Reece and R. A. Lukaszew, "Niobium thin film deposiiton studies on copper surfaces for superconducting radio frequency cavity applications," Physical Review Special Topics - Accelerators and Beams, vol. 15, p. 062002, 2012.

[69] A. Anders, R. J. Mendelsberg, S. Lim, M. Mentink, J. L. Slack, J. G. Wallig, A. V. Nollau and G. Y. Yushkov, "Deposition of Niobium and Other Superconducting Materials with High Power Impulse Magnetron Sputtering: Concep and First Results," in Proceedings of SRF 2011, Chigaco, IL USA, 2011.

[70] K. Makise, H. Terai, M. Taked, Y. Uzawa and Z. Wang, "Characterization of NbTiN Thin Films Deposited on Various Substrates," IEEE TRANSACTIONS ON APPLIED SUPERCONDUCTIVITY, vol. 21, no. 3, pp. 139-142, 2011.

[71] A. Gustafsson, S. Calatroni, W. Vollenberg and R. Seviour, "New Methods for Thin Film Deposition and First Investigations of the Use of High Temperature 
Superconductors for Thin Film Cavities," in Proceedings of IPAC'10, Kyoto, 2010.

[72] T. Shiino, S. Shiba, N. Sakai, T. Yamakura, L. Jian, Y. Uzawa, H. Maezawa and S. Yamamoto, "Improvement of the Critical Temperature of Superconducting NbTiN and NbN Thin Films Using the AIN Buffer Layer," Superconducting Science and Technology, vol. 23, no. 4, p. 045004, 2010.

[73] A. Anders, "High power impulse magnetron sputtering and related discharges: Scalable plasma sources for plasma-based ion implantation and deposition," Surface \& Coatings Technology, vol. 204, no. 18-19, pp. 28642868, 2010.

[74] M. Lattemann, U. Helmersson and J. E. Greene, "Fully Dense, Non-Faceted 111-Textured High Power Impulse Magnetron Sputtering TiN Films Grown in the Absence of Substrate Heating and Bias," Thin Solid Films, vol. 518, no. 21, pp. 5978-5980, 2010.

[75] A. P. Ehiasarian, J. Andersson and A. Anders, "Distance-dependent plasma composition and ion energy in high power impulse magnetron sputtering," Journal of Physics D: Applied Physics, vol. 43, p. 275204, 2010. 
[76] A. Anders, "Deposition Rates of High Power Impulse Magnetron Sputtering: Physics and Economics," Journal of Vacuum Science and Technology A, vol. 28, no. 783, 2010.

[77] D. Lundin, N. Brenning, D. Jadernas, P. Larsson, E. Wallin, M. Lattemann, M. A. Raddu and U. Helmersson, "Transition betweeen the discharge regimes of high power impulse magnetron sputtering and conventional direct current magnetron sputtering," Plasma Sources Science and Technology, vol. 18, no. 4, p. 045008, 2009.

[78] A. Anders and E. Oks, "Evolution of the plasma composition of a high power impulse magnetron sputtering system studied with a time-of-flight spectrometer," Journal of Applied Physics, vol. 150, no. 9, p. 093304, 2009.

[79] M. Wen, C. Q. Hu, Q. N. Meng, Z. D. Zhao, T. An, Y. D. Su, W. X. Yu and W. T. Zheng, "Effects of nitrogen flow rate on the preferred orientation and phase transition for niobium nitride films grown by direct current reactive magnetron sputtering," Journal of Physics D: Applied Physics, vol. 42, p. 035304, 2009.

[80] J. J. Olaya, S. E. Rodil and S. Muhl, "Comparative study of niobium nitride coatings deposited by unbalanced and balanced magnetron sputtering," Thin Solid Films, vol. 516, pp. 8319-8326, 2008. 
[81] N. Brenning, I. Axnas, M. A. Raadu, D. Lundin and U. Helmersson, "A bulk plasma model for DC and HiPIMS magnetrons," Plasma Sources Science and Technology, vol. 17, no. 4, p. 045009, 2008.

[82] A. Anders, J. Andersson, D. Horwat and A. Ethiasarian, "Physics of High Power Impulse Magnetron Sputtering," in The Ninth Inernational Symposium on Sputtering \& Plasma Processes-ISSP, 2007.

[83] J. Bohlmark, M. Lattemann, J. T. Gudmundsson, A. P. Ehiasarian, Y. A. Gonzalvo, N. Brenning and U. Helmersson, "The ion energy distributions and ion flux composition from a high power impulse magnetron sputtering discharge," Thin Solid Films, vol. 515, no. 4, pp. 1522-1526, 2006.

[84] J. Bohlmark, "Fundamentals of High Power Impulse Magnetron Sputtering," 2006.

[85] C. S. Sandu, R. Sanjines, M. Benkahoul, F. Medjani and F. Levy, "Formation of composite ternary nitride thin films by magnetron sputtering codeposition," Surface \& Coatings Technology, vol. 201, pp. 4083-4089, 2006.

[86] J. Musil, P. Baroch, J. Vlcek, K. H. Nam and J. G. Han, "Reactive magnetron sputtering of thin films: present status and trends," Thin Solid Films, vol. 475, pp. 208-218, 2005. 
[87] M. Benkahoul, "Niobium nitride based thin films deposited by DC reactive magnetron sputtering: NbN, NbSiN and NbAIN," Escola Politécnica Federal de Lausanne (École Polytechnique Fédérale de Lausanne), Lausanne: Tese (Doutorado), 2005.

[88] L. Yu, R. K. Singh, H. Liu, S. Y. Wu, R. Hu, D. Durand, J. Bulman, J. M. Rowell and N. Newman, "Fabrication of Niobium Titanium Nitride Thin Films with High Superconducting Transition Temperatures and Short Penetration Lengths," IEEE TRANSACTIONS ON APPLIED SUPERCONDUCTIVITY, vol. 15, no. 1, pp. 44-48, 2005.

[89] L. You, R. K. Singh, S. Y. Wu, R. Hu, D. Durand, J. Bulman, J. M. Rowell and N. Newman, "Fabrication of Niobium Titanium Nitride Thin Films with High Superconducting Transition Temperatures and Short Penetration Lengths," IEEE Transactions on Applied Superconductivity, vol. 15, no. 1, pp. 44-48, 2005.

[90] W. D. Sproul, D. J. Christie and D. C. Carter, "Control of reactive sputtering processes," Thin Solid Films, vol. 491, pp. 1-17, 2005.

[91] T. Matsunaga, H. Maezawa and T. Noguchi, "Characterization of NbTiN Thin Films Prepared by Reactive DC-Magnetron Sputtering," IEEE TRANSACTIONS ON APPLIED SUPERCONDUCTIVITY, vol. 13, no. 2, pp. 3284-3287, 2003. 
[92] J. Y. Rauch, C. Rousselot and N. Martin, "Structure and composition of TixAl1$\mathrm{xN}$ thin films sputter deposited using a composite metallic target," Surface and Coatings Technology, vol. 157, pp. 138-143, 2002.

[93] E. Wahlstrom, I. Ekvall, T. Kihlgren, H. Olin, S. Lindgren and L. Wallden, "Lowtemperature structure of S/Cu(111)," Physical Review B, vol. 64, p. 155406, 2001.

[94] K. Macak, V. Kouznetsov, J. Schneider and U. Helmersson, "Ionized sputter deposition using an extremely hihg plasma density pulsed magnetron discharge," Journal of Vacuum Science and Technology A, vol. 18, no. 4, 2000.

[95] K. Macak, V. Kouznetsov, J. Schneider and U. Helmersson, "Ionized Sputter Deposition Using an Extremely High Plasma Density Pulsed Magnetron Discharge," Journal of Vacuum Science and Technology A, vol. 18, no. 4, pp. 1533-1537, 2000. 